\title{
Divisão de distribuições temperadas por polinốmios
}

\author{
Mariana Smit Vega Garcia
}

\author{
DISSERTAÇÃO APRESENTADA \\ $\mathrm{AO}$ \\ Instituto DE MATEMÁtica E Estatística \\ Universidade DE SÃo PAUlo \\ PARA \\ OBTENÇẪO DO TÍTULO \\ Mestre EM CiÊnCIAS \\ Área de Concentração: Matemática \\ Orientador: Prof. Dr. Paulo Domingos Cordaro
}

Durante o desenvolvimento deste trabalho a autora recebeu auxílio financeiro da FAPESP

São Paulo, julho de 2008 


\section{Divisão de distribuições temperadas por polinômios}

Este exemplar corresponde à redação final da dissertação devidamente corrigida e defendida por Mariana Smit Vega Garcia e aprovada pela Comissão Julgadora.

Banca Examinadora:

- Prof. Dr. Paulo Domingos Cordaro (orientador) - IME-USP.

- Prof. Dr. Jorge Manuel Sotomayor Tello - IME-USP.

- Prof. Dr. Gerson Petronilho - UFSCar. 


\section{Agradecimentos}

Gostaria de agradecer ao Professor Paulo Cordaro toda a ajuda e inspiração que estiveram sempre presentes, não somente durante o mestrado, como também durante minha graduação.

Cabe ainda agradecer à minha mãe a paciência e a compreensão durante este processo, e ao meu pai os conselhos.

Finalmente, gostaria de agradecer ao Rodrigo, que sempre esteve presente me ajudando e incentivando ao longo da minha jornada pela matemática.

Voor Moma, van jouw meissie. 


\section{Resumo}

Este trabalho apresenta uma demonstração completa do Teorema de L. Hörmander sobre a divisão de distribuições (temperadas) por polinômios. O caso $n=1$ é apresentado detalhadamente e serve como motivação para as técnicas utilizadas no caso geral. Todos os pré-requisitos para a demonstração de Hörmander (os Teoremas de Seidenberg-Tarski, de Puiseux e da Extensão de Whitney) são discutidos com detalhes. Como conseqüência do Teorema, segue que todo operador diferencial parcial linear com coeficientes constantes não nulo admite solução fundamental temperada.

Palavras-chave: Distribuições, divisão, polinômios, distribuições temperadas. 


\section{Abstract}

This dissertation presents a thorough proof of L. Hörmander's theorem on the division of (tempered) distributions by polynomials. The case $n=1$ is discussed in detail and serves as a motivation for the techniques that are utilised in the general case. All the prerequisites for Hörmander's proof (the Theorems of Seidenberg-Tarski, of Puiseux and Whitney's Extension Theorem) are discussed in detail. As a consequence of this theorem, it follows that every non zero partial differencial operator with constant coefficients has a tempered fundamental solution.

Keywords: Distributions, division, polynomials, tempered distributions. 


\section{Sumário}

1 Introdução 11

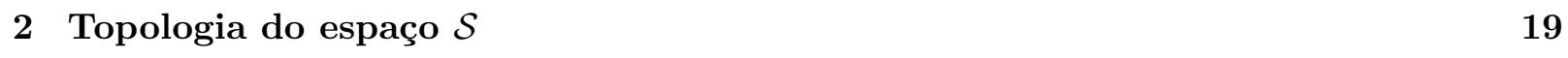

$\begin{array}{lll}3 & \text { Teorema de Seidenberg-Tarski } & 23\end{array}$

4 Séries de Puiseux $\quad 29$

5 Lema algébrico $\quad 39$

6 Teorema da Extensão de Whitney $\quad 45$

$\begin{array}{lll}7 & \text { Prova do Teorema principal } & 63\end{array}$

8 Apêndice $\quad 85$

9 Notações $\quad 93$

$\begin{array}{ll}\text { Referências Bibliográficas } & 95\end{array}$ 


\section{Capítulo 1}

\section{Introdução}

Após o desenvolvimento da Teoria das Distribuições por Schwartz em 1950 surgiu a seguinte questão: dados um aberto $\Omega \subseteq \mathbb{R}^{n}$, uma função $f \in \mathcal{C}^{\infty}(\Omega)$ e uma distribuição $S \in \mathcal{D}^{\prime}(\Omega)$, existe uma distribuição $T \in \mathcal{D}^{\prime}(\Omega)$ tal que $f T=S$ ?

Esse resultado é falso, como se pode ver considerando, por exemplo, uma função $f$ que se anula em um aberto $U \subseteq \Omega$ não vazio e uma distribuição $S$ que não se anula nesse aberto.

$\mathrm{Na}$ verdade, se $f$ se anular somente em um ponto de $\Omega$ a resposta continua sendo, em geral, negativa. Vejamos um exemplo. Consideremos a função $f \in \mathcal{C}^{\infty}(\mathbb{R})$ definida a seguir:

$$
f(x)=\left\{\begin{array}{r}
e^{-\frac{1}{x^{2}}}, \text { se } x \neq 0 \\
0, \text { se } x=0
\end{array}\right.
$$

Suponhamos por absurdo que existe uma distribuição $T \in \mathcal{D}^{\prime}(\mathbb{R})$ tal que $f T=1$. Sejam $C>0$ e $N \in \mathbb{N}$ tais que

$$
|T(\varphi)| \leq C \sum_{j=0}^{N} \sup _{x \in[-1,1]}\left|\varphi^{(j)}(x)\right|, \forall \varphi \in \mathcal{C}_{c}^{\infty}([-1,1]) .
$$

Seja $\varphi \in \mathcal{C}_{c}^{\infty}(] 0,1[)$. Então

$$
T(\varphi)=T\left(e^{-\frac{1}{x^{2}}} e^{\frac{1}{x^{2}}} \varphi\right)=e^{\frac{-1}{x^{2}}} T\left(e^{\frac{1}{x^{2}}} \varphi\right)=1\left(e^{\frac{1}{x^{2}}} \varphi\right)=\int e^{\frac{1}{x^{2}}} \varphi(x) d x .
$$

Conseqüentemente,

$$
\left|\int e^{\frac{1}{x^{2}}} \varphi(x) d x\right| \leq C \sum_{j=0}^{N} \sup _{x \in[-1,1]}\left|\varphi^{(j)}(x)\right| .
$$

Para cada $n \in \mathbb{N}$, seja $\varphi_{n} \in \mathcal{C}_{c}^{\infty}(] 0,1[)$ tal que $0 \leq \varphi_{n} \leq 1, \varphi_{n}=1$ em uma vizinhança de $[1 /(n+2), 1 /(n+1)]$ e, além disso, para todo $m \in \mathbb{N}$

$$
\left\|\varphi_{n}^{(m)}\right\|_{\infty}=\mathrm{O}\left(n^{m}\right)
$$

Podemos afirmar, então, que existe uma constante $K>0$ tal que

$$
\int e^{\frac{1}{x^{2}}} \varphi_{n}(x) d x \leq K \cdot n^{N}
$$


Entretanto

$$
\int e^{\frac{1}{x^{2}}} \varphi_{n}(x) d x \geq \int_{\frac{1}{n+2}}^{\frac{1}{n+1}} e^{\frac{1}{x^{2}}} d x \geq e^{(n+1)^{2}} \frac{1}{(n+1)(n+2)}
$$

Dessas duas últimas afirmações concluiríamos que existe uma constante $K^{\prime}$ tal que $e^{(n+1)^{2}} \leq$ $K^{\prime} n^{N+2}, \forall n \in \mathbb{N}$, o que não é possível.

Continuando com a história do problema da divisão, em 1932 Hadamard estudou as seguintes funções definidas em $\mathbb{R}$, onde $a \in \mathbb{C}$ :

$$
x_{+}^{a}=\left\{\begin{aligned}
x^{a}, & \text { se } x \geq 0, \\
0, & \text { se } x<0 .
\end{aligned}\right.
$$

Pode-se provar que se $\operatorname{Re}(a)>-1$, então $x_{+}^{a} \in L_{\text {loc }}^{1}(\mathbb{R}) \subseteq \mathcal{D}^{\prime}(\mathbb{R})$. Quanto aos outros casos, através de continuação analítica é possível dar significado para $x_{+}^{a}$, para todo $a \in \mathbb{C}$ (como por exemplo para $\left.x_{+}^{-1}\right)$.

Em 1956 Schwartz resolveu o problema da divisão se $\Omega \subseteq \mathbb{C}^{n}$ e $f=f\left(z_{1}, \ldots, z_{n}\right)$ é uma função não nula e holomorfa em cada uma de suas variáveis. Tal resultado encontra-se em [10].

Pouco tempo depois, em 1958, Lojasiewicz provou em [9] que o resultado é verdadeiro se $f$ for uma função real analítica. No mesmo período Hörmander provou em [7] que o resultado vale no caso em que $f$ é um polinômio; embora o resultado de Lojasiewicz implique o de Hörmander, a prova do último é consideravelmente mais simples e envolve técnicas muito interessantes. Do Teorema de Hörmander segue que todo operador diferencial parcial linear não nulo de coeficientes constantes admite solução fundamental temperada.

Ambas as provas de Lojasiewicz e de Hörmander se baseiam na seguinte desigualdade fundamental: dada $Q$ uma função não nula (para Hörmander será um polinômio, para Lojasiewicz uma função real analítica), seja $\mathcal{N}$ o conjunto de seus zeros. Então existem constantes $c, \mu>0$ tais que

$$
c \operatorname{dist}(\xi, \mathcal{N})^{\mu} \leq|Q(\xi)|, \forall \xi \in \mathbb{R}^{n}:|\xi| \leq 1 .
$$

Hörmander provou esse resultado utilizando o Teorema de Seidenberg-Tarski enquanto Lojasiewicz conseguiu prová-lo para funções reais analíticas após um estudo detalhado da estratificação do conjunto de zeros de $Q$.

Finalmente, Atiyah provou em 1970 que é possível dividir distribuições por polinômios através da Resolução de Singularidades (o leitor mais interessado pode consultar [1]).

Depois dessa introdução histórica, vale relembrar que o principal objetivo desse trabalho é provar o seguinte teorema:

Teorema 1.0.1 Sejam $P(D)$ um operador diferencial parcial com coeficientes constantes não nulo e $T$ uma distribuição temperada. Então existe uma distribuição temperada $S$ tal que

$$
P(D) S=T .
$$

Em particular, sempre existe solução fundamental temperada de $P(D)$. 
Para provar esse resultado utilizaremos outro teorema, cuja prova ocupará o resto do trabalho. Para enunciá-lo vamos introduzir a seguinte definição: diremos que uma função $\varphi \in \mathcal{C}^{\infty}\left(\mathbb{R}^{n}\right)$ pertence ao espaço de Schwartz, denotado por $\mathcal{S}$, se, e somente se,

$$
\sup _{x \in \mathbb{R}^{n}}\left|x^{\alpha} \partial^{\beta} \varphi(x)\right|<\infty, \forall \alpha, \beta \in \mathbb{N}^{n} .
$$

Trataremos brevemente da topologia desse espaço no capítulo seguinte.

O enunciado do teorema mencionado acima é:

Teorema 1.0.2 Seja $P$ um polinômio não-nulo em n variáveis. Então a aplicação multiplicação

$$
\mathcal{S} \ni f \longmapsto P f \in \mathcal{S}
$$

tem uma inversa contínua, isto é, dada uma semi-norma contínua $\mathcal{R}$ definida em $\mathcal{S}$ existe uma semi-norma contínua $\mathcal{Q}$ definida em $\mathcal{S}$ tal que $\mathcal{R}(f) \leq \mathcal{Q}(P f), \forall f \in \mathcal{S}$.

Vejamos algumas conseqüências desse teorema.

Teorema 1.0.3 Sejam $T$ uma distribuição temperada e $P$ um polinômio não nulo em n variáveis. Então existe uma distribuição temperada $S$ tal que

$$
P S=T \text {. }
$$

Demonstração: Consideremos a aplicação linear definida no subespaço $P \mathcal{S} \subseteq \mathcal{S}$ dada por

$$
P \mathcal{S} \ni P f \longmapsto T(f) .
$$

Pelo teorema 1.0.2 essa aplicação é contínua: de fato, se $P f \rightarrow 0$ em $\mathcal{S}$ então $f \rightarrow 0$ em $\mathcal{S}$, e portanto $T(f) \rightarrow 0$. Pelo Teorema de Hahn-Banach podemos estender a aplicação acima a uma aplicação linear contínua $S: \mathcal{S} \rightarrow \mathbb{C}$ definida em todo o espaço $\mathcal{S}$. Mas então

$$
P S(f)=S(P f)=T(f), \forall f \in \mathcal{S}
$$

e portanto $P S=T$.

Teorema 1.0.4 Sejam $T$ uma distribuição definida num aberto $\Omega \subseteq \mathbb{R}^{n}$ e $P$ um polinômio não nulo em $n$ variáveis. Então existe uma distribuição $S$ definida em $\Omega$ tal que

$$
P S=T .
$$

Demonstração: Vejamos inicialmente que dado um ponto $x_{0} \in \Omega$ existem uma vizinhança $U$ de $x_{0}$ em $\Omega$ e $S_{0} \in \mathcal{D}^{\prime}(U)$ tais que $P S_{0}=T$ em $U$. De fato, sejam $U \subseteq \Omega$ um aberto com $x_{0} \in U$ e $\phi \in \mathcal{C}_{c}^{\infty}(\Omega)$ tal que $\phi=1$ em $U$. Então $\phi T \in \mathcal{S}^{\prime}$ e portanto, pelo teorema anterior, existe $R \in \mathcal{S}^{\prime}$ tal que $P R=\phi T$. Seja $S_{0}=\left.R\right|_{\mathcal{C}_{c}^{\infty}(U)}$. Assim $S_{0} \in \mathcal{D}^{\prime}(U)$ e $P S_{0}=\phi T=T$ em $U$.

Vamos considerar agora uma cobertura enumerável $\left\{U_{m}\right\}_{m \in \mathbb{N}}$ de $\Omega$ tais que:

1. Cada aberto $U_{m}$ é aberto relativamente compacto em $\Omega$.

2. A cobertura $\left\{U_{m}\right\}_{m \in \mathbb{N}}$ é localmente finita. 
3. Para cada $m \in \mathbb{N}$ existe $S_{m} \in \mathcal{D}^{\prime}\left(U_{m}\right)$ tal que $P S_{m}=T$ em $U_{m}$.

Sejam $\phi_{m} \in \mathcal{C}_{c}^{\infty}\left(U_{m}\right)$ tais que $\sum_{m=1}^{\infty} \phi_{m}=1$. Definamos

$$
S=\sum_{m=1}^{\infty} \phi_{m} S_{m}
$$

Temos que

$$
P S=\sum_{m=1}^{\infty}\left(\phi_{m} P S_{m}\right)=\left(\sum_{m=1}^{\infty} \phi_{m}\right) T=T .
$$

Vejamos agora a prova do teorema 1.0.1.

Demonstração: Sejam $P(D)$ um operador linear parcial diferencial com coeficientes constantes e $T$ uma distribuição temperada. Queremos encontrar uma distribuição temperada $S$ tal que

$$
P(D) S=T .
$$

Se tal distribuição temperada existir, aplicando a transformada de Fourier na equação acima obtemos:

$$
P(\xi) \hat{S}=\hat{T},
$$

onde $\hat{S}$ e $\hat{T}$ denotam as transformadas de Fourier de $S$ e de $T$. Pelo teorema 1.0.3 essa equação tem uma solução $\hat{S}$, uma distribuição temperada. Invertendo a transformada de Fourier obtemos nossa solução $S$.

Agora vamos dar uma idéia dos passos necessários para provar o teorema 1.0.2. A prova desse teorema será feita por indução, mas começaremos por provar um caso particular (o que será feito sem utilizar a hipótese de indução). No momento em que formos provar o passo da indução precisaremos do caso particular e de um outro teorema importante, o Teorema da Extensão de Whitney, que será provado no capítulo 6. Para provarmos esse teorema utilizaremos o seguinte lema, que será provado no capítulo 5:

Lema 1.0.1 Sejam $Q$ um polinômio em $\mathbb{R}^{n}$ e $\mathcal{N}$ o conjunto dos seus zeros. Então ou $\mathcal{N}=\emptyset e$ existem constantes $C, a>0$ tais que

$$
C\left(1+|\xi|^{2}\right)^{-a} \leq|Q(\xi)|, \forall \xi \in \mathbb{R}^{n},
$$

ou $\mathcal{N} \neq \emptyset$ e existem constantes $C, a, b>0$ tais que

$$
C\left(1+|\xi|^{2}\right)^{-a} \operatorname{dist}(\xi, \mathcal{N})^{b} \leq|Q(\xi)|, \forall \xi \in \mathbb{R}^{n} .
$$

A prova desse resultado utiliza, por sua vez, os Teoremas de Seidenberg-Tarski e de Puiseux, que aparecem nos capítulos 3 e 4 . No capítulo 2 vamos estudar um pouco mais a topologia do espaço $\mathcal{S}$, o que será útil no restante do trabalho. No capítulo 9 se encontra uma breve descrição das notações utilizadas durante este trabalho. 
Para finalizar esse capítulo, vale a pena descrever a prova do teorema 1.0.2 no caso $n=1$.

Para prová-lo devemos mostrar que se $P$ é um polinômio real e $m, p \in \mathbb{N}$, existem $k \in \mathbb{N}$, $C>0$, constantes $m_{i} \in \mathbb{N}$, para $0 \leq i \leq k$ tais que

$$
\sup _{x \in \mathbb{R}}\left|x^{m} D^{p} f(x)\right| \leq C \sum_{i=0}^{k} \sup _{x \in \mathbb{R}}\left|x^{m_{i}} D^{i}(P f)(x)\right|, \forall f \in \mathcal{S} .
$$

Comecemos a prova com um caso mais simples.

Lema 1.0.2 Dado $p \in \mathbb{N}$, existem $q \in \mathbb{N}, C>0$ tais que

$$
\sup _{x \in \mathbb{R}}\left|D^{p} f(x)\right| \leq C \sum_{j=0}^{q} \sup _{x \in \mathbb{R}}\left|D^{j}(P f)(x)\right|, \forall f \in \mathcal{S} .
$$

Demonstração: Vejamos inicialmente que o resultado é verdadeiro se $P(x)=(x-a)^{r}$, onde $a \in \mathbb{R}, r \geq 1$. Escrevendo a fórmula de Taylor da função $g(x)=f(x)(x-a)^{r}$ no ponto $a$ de ordem $r-1$ teremos que

$$
(x-a)^{r} f(x)=\int_{0}^{1} \frac{(1-t)^{r-1}}{(r-1) !} D^{r}\left\{(x-a)^{r} f(x)\right\}(a+t(x-a)) \cdot(x-a)^{r} d t,
$$

e portanto

$$
f(x)=\int_{0}^{1} \frac{(1-t)^{r-1}}{(r-1) !} D^{r}\left\{(x-a)^{r} f(x)\right\}(a+t(x-a)) d t .
$$

Após derivarmos essa expressão vemos que existe uma constante $C$ (independente de $f$ ) tal que

$$
\left|D^{p} f(x)\right| \leq C \sup _{y \in \mathbb{R}}\left|D^{r+p}(P f)(y)\right|
$$

Dado $P$ um polinômio qualquer, vamos escrevê-lo como

$$
P(x)=q(x) \cdot\left(x-x_{1}\right)^{r_{1}} \ldots . .\left(x-x_{l}\right)^{r_{l}},
$$

onde $q(x) \neq 0, \forall x \in \mathbb{R}, x_{i} \in \mathbb{R}$ e $r_{i} \geq 1$. Seja $r>0$ tal que $B_{r}\left[x_{i}\right] \cap B_{r}\left[x_{j}\right]=\emptyset$, se $i \neq j$. Sejam $\varphi_{i} \in \mathcal{C}_{c}^{\infty}(\mathbb{R})$ tais que $\operatorname{supp} \varphi_{i} \subseteq B_{r}\left[x_{i}\right]$ e $\varphi_{i}(x)=1, \forall x \in B_{\frac{r}{2}}\left[x_{i}\right]$. Temos que

$$
\begin{aligned}
f & =f \varphi_{1}+\left(1-\varphi_{1}\right) f \\
& =f \varphi_{1}+\left(1-\varphi_{1}\right) \varphi_{2} f+\left(1-\varphi_{1}\right)\left(1-\varphi_{2}\right) f \\
& =f \varphi_{1}+\left(1-\varphi_{1}\right) \varphi_{2} f+\ldots+\left(1-\varphi_{1}\right) \ldots\left(1-\varphi_{l}\right) f .
\end{aligned}
$$

Derivando teremos que

$$
D^{p} f=D^{p}\left(f \varphi_{1}\right)+D^{p}\left(\left(1-\varphi_{1}\right) \varphi_{2} f\right)+\ldots+D^{p}\left(\left(1-\varphi_{1}\right) \ldots\left(1-\varphi_{l}\right) f\right) .
$$


Por (1.0.1), existe uma constante $C_{1}>0$, independente de $f$, tal que

$$
\begin{aligned}
\left|D^{p}\left(f \varphi_{1}\right)(x)\right| & \leq C_{1} \sup _{y \in \mathbb{R}}\left|D^{p+r_{1}}\left\{\left(y-x_{1}\right)^{r_{1}} \varphi_{1}(y) f(y)\right\}\right| \\
& =C_{1} \sup _{y \in \mathbb{R}}\left|D^{p+r_{1}}\left\{\frac{\varphi_{1}(y)}{q(y)\left(y-x_{2}\right)^{r_{2}} \ldots\left(y-x_{l}\right)^{r_{l}}} P(y) f(y)\right\}\right| \\
& \leq C_{1}^{\prime} \sup _{y \in \mathbb{R}} \sum_{j=0}^{p+r_{1}}\left|D^{j}(P f)(y)\right| \\
& \leq C_{1}^{\prime} \sum_{j=0}^{p+r_{1}} \sup _{y \in \mathbb{R}}\left|D^{j}(P f)(y)\right|
\end{aligned}
$$

Tratamos analogamente todos os termos, menos $D^{p}\left\{\left(1-\varphi_{1}\right) \ldots\left(1-\varphi_{l}\right) f\right\}$. Existe uma constante $C_{0}>0$ tal que

$$
\begin{aligned}
\sup _{x \in \mathbb{R}}\left|D^{p}\left\{\left(1-\varphi_{1}\right) \ldots\left(1-\varphi_{l}\right) f(x)\right\}\right| & =\sup _{x \in \mathbb{R}}\left|D^{p}\left\{\frac{\left(1-\varphi_{1}(x)\right) \ldots\left(1-\varphi_{l}(x)\right)}{P(x)}(P f)(x)\right\}\right| \\
& \leq C_{0} \sum_{j=0}^{p} \sup _{y \in \mathbb{R}}\left|D^{j}(P f)(y)\right| .
\end{aligned}
$$

Concluimos que existe uma constante $C>0$ (independente de $f$ ) tal que se $s=r_{1}+\ldots+r_{l}$

$$
\sup _{x \in \mathbb{R}}\left|D^{p} f(x)\right| \leq C \sum_{j=0}^{p+s} \sup _{y \in \mathbb{R}}\left|D^{j}(P f)(y)\right| .
$$

Falta provar o caso geral, isto é, dados $m, p \in \mathbb{N}$, existem $k \in \mathbb{N}, C>0$ e constantes $m_{i} \in \mathbb{N}$, com $0 \leq i \leq k$, tais que

$$
\sup _{x \in \mathbb{R}}\left|x^{m} D^{p} f(x)\right| \leq C \sum_{i=0}^{k} \sup _{x \in \mathbb{R}}\left|x^{m_{i}} D^{i}(P f)(x)\right|, \forall f \in \mathcal{S} .
$$

Faremos o caso $m=1$, pois para provar o caso geral basta utilizar a mesma idéia e a hipótese de que o fato já foi provado para os $m$ menores. Temos que

$$
x D^{p} f(x)=D^{p}(x f)-p D^{p-1} f(x) .
$$

Assim, existem constantes $C_{1}, C_{2}>0$ tais que se $x \in \mathbb{R}$,

$$
\begin{aligned}
\left|x D^{p} f(x)\right| & \leq\left|D^{p}(x f)\right|+\left|p D^{p-1} f(x)\right| \\
& \leq C_{1} \sum_{j=0}^{p+s} \sup _{y \in \mathbb{R}}\left|D^{j}(P x f)(y)\right|+C_{2} \sum_{i=0}^{p-1+s} \sup _{y \in \mathbb{R}}\left|D^{i}(P f)(y)\right| \\
& \leq C_{1} \sum_{j=0}^{p+s} \sup _{y \in \mathbb{R}}\left(\left|j D^{j-1}(P f)(y)\right|+\left|y D^{j}(P f)(y)\right|\right)+C_{2} \sum_{i=0}^{p-1+s} \sup _{y \in \mathbb{R}}\left|D^{i}(P f)(y)\right|,
\end{aligned}
$$


e segue o resultado.

Finalmente apresentamos uma descrição das referências bibliográficas utilizadas na elaboração deste trabalho.

O capítulo 3 é baseado no Apêndice A de [6]. O capítulo 4 é baseado em [8], com uma nova demonstração do Teorema 4.0.7 (Lema de Hensel), além do Apêndice A de [6]. O restante da dissertação é quase que todo baseado em [7], com exceção do Teorema 6.0.14, que foi baseado em [11], sofrendo algumas alterações adicionais.

Ao longo deste trabalho procuramos apresentar o assunto com o maior número possível de detalhes visando, principalmente, tornar claro ao leitor menos experiente este assunto tão interessante. 


\section{Capítulo 2}

\section{Topologia do espaço $\mathcal{S}$}

Vamos começar relembrando alguns fatos que podem ser interessantes para o leitor.

Definição 2.0.1 Uma função $\varphi \in \mathcal{C}^{\infty}\left(\mathbb{R}^{n}\right)$ pertence ao espaço de Schwartz, denotado por $\mathcal{S}$, se, e somente se,

$$
\sup _{x \in \mathbb{R}^{n}}\left|x^{\alpha} \partial^{\beta} \varphi(x)\right|<\infty, \forall \alpha, \beta \in \mathbb{N}^{n} .
$$

Lema 2.0.3 Para todo $\alpha \in \mathbb{N}^{n}$, temos que $\left|x^{\alpha}\right| \leq|x|^{|\alpha|}, \forall x \in \mathbb{R}^{n}$.

Demonstração: De fato, se $x=\left(x_{1} \ldots, x_{n}\right)$ e $\alpha=\left(\alpha_{1}, \ldots, \alpha_{n}\right)$,

$$
\left|x^{\alpha}\right|=\left|x_{1}^{\alpha_{1}} \ldots x_{n}^{\alpha_{n}}\right|=\left|x_{1}\right|^{\alpha_{1}} \ldots\left|x_{n}\right|^{\alpha_{n}} \leq|x|^{\alpha_{1}} \ldots|x|^{\alpha_{n}}=|x|^{|\alpha|} .
$$

Lema 2.0.4 Valem as seguintes afirmações, para $\varphi \in \mathcal{C}^{\infty}\left(\mathbb{R}^{n}\right)$ :

1. $\varphi \in \mathcal{S}$ se, e somente se, $\lim _{|x| \rightarrow \infty}\left|x^{\alpha} \partial^{\beta} \varphi(x)\right|=0, \forall \alpha, \beta \in \mathbb{N}^{n}$.

2. $\varphi \in \mathcal{S}$ se, e somente se, $\sup _{|\beta| \leq m} \sup _{x \in \mathbb{R}^{n}}\left(1+|x|^{2}\right)^{m}\left|\partial^{\beta} \varphi(x)\right|<\infty, \forall m \in \mathbb{N}$.

Demonstração: Provemos que se $\lim _{|x| \rightarrow \infty}\left|x^{\alpha} \partial^{\beta} \varphi(x)\right|=0, \forall \alpha, \beta \in \mathbb{N}^{n}$, então $\varphi \in \mathcal{S}$. Dados $\alpha, \beta \in \mathbb{N}^{n}$, existe $R>0$ tal que

$$
\left|x^{\alpha} \partial^{\beta} \varphi(x)\right|<1, \forall x:|x|>R
$$

Como $\sup _{|x| \leq R}\left|x^{\alpha} \partial^{\beta} \varphi(x)\right|=A<\infty$, então é claro que $\varphi \in \mathcal{S}$.

Quanto ao outro lado da equivalência, sejam $\alpha, \beta \in \mathbb{N}^{n}$. Para cada $1 \leq j \leq n$, existe $C_{j}=C_{j, \alpha, \beta}>0$ tal que

$$
\left|x^{\alpha} x_{j}^{2} \partial^{\beta} \varphi(x)\right| \leq C_{j}, \forall x \in \mathbb{R}^{n} ;
$$

assim,

$$
|x|^{2}\left|x^{\alpha} \partial^{\beta} \varphi(x)\right| \leq C_{1}+\ldots+C_{n}=C,
$$

e portanto $\left|x^{\alpha} \partial^{\beta} \varphi(x)\right| \leq C /|x|^{2}, \forall x \in \mathbb{R}^{n}, x \neq 0$. Concluimos que $\lim _{|x| \rightarrow \infty}\left|x^{\alpha} \partial^{\beta} \varphi(x)\right|=0$.

Provemos 2. Vejamos que se $\sup _{|\beta| \leq m} \sup _{x \in \mathbb{R}^{n}}\left(1+|x|^{2}\right)^{m}\left|\partial^{\beta} \varphi(x)\right|<\infty, \forall m \in \mathbb{N}$, então $\varphi \in \mathcal{S}$. Sejam $\alpha, \beta \in \mathbb{N}^{n}$. Podemos assumir que $|x|>1$ pois $\sup _{|x| \leq 1}\left|x^{\alpha} \partial^{\beta} \varphi(x)\right|<\infty$. Definamos $|\alpha|+|\beta|=m$. 


$$
\begin{aligned}
\left|x^{\alpha} \partial^{\beta} \varphi(x)\right| & \leq|x|^{|\alpha|}\left|\partial^{\beta} \varphi(x)\right| \\
& \leq|x|^{2|\alpha|}\left|\partial^{\beta} \varphi(x)\right| \\
& \leq\left(1+|x|^{2}\right)^{|\alpha|}\left|\partial^{\beta} \varphi(x)\right| \\
& \leq\left(1+|x|^{2}\right)^{|\alpha|+|\beta|}\left|\partial^{\beta} \varphi(x)\right| \\
& \leq \sup _{y \in \mathbb{R}^{n}}\left(1+|y|^{2}\right)^{m}\left|\partial^{\beta} \varphi(y)\right| \\
& \leq \sup _{|\gamma| \leq m} \sup _{y \in \mathbb{R}^{n}}\left(1+|y|^{2}\right)^{m}\left|\partial^{\gamma} \varphi(y)\right|=A<\infty .
\end{aligned}
$$

Assim $\left|x^{\alpha} \partial^{\beta} \varphi(x)\right| \leq A<\infty, \forall x \in \mathbb{R}^{n}:|x|>1$. Quanto ao outro lado da desigualdade, vamos assumir que $\varphi \in \mathcal{S}$. Sejam $m \in \mathbb{N}$ e $\beta \in \mathbb{N}^{n}$ tal que $|\beta| \leq m$.

$$
\sup _{x \in \mathbb{R}^{n}}\left(1+|x|^{2}\right)^{m}\left|\partial^{\beta} \varphi(x)\right|=\sup _{x \in \mathbb{R}^{n}} \sum_{j=0}^{m}\left(\begin{array}{c}
m \\
j
\end{array}\right)|x|^{2 j}\left|\partial^{\beta} \varphi(x)\right| \leq \sum_{j=0}^{m}\left(\begin{array}{c}
m \\
j
\end{array}\right) \sup _{x \in \mathbb{R}^{n}}|x|^{2 j}\left|\partial^{\beta} \varphi(x)\right| .
$$

Mas para cada $0 \leq j \leq m,|x|^{2 j}=\left(x_{1}^{2}+\ldots+x_{n}^{2}\right)^{j}$ é uma soma de termos do tipo $c\left|x^{\gamma}\right|$, para certos $\gamma \in \mathbb{N}^{n}$ e constantes $c$. Como $\varphi \in \mathcal{S}$, então é claro que $\sup _{x \in \mathbb{R}^{n}}\left(1+|x|^{2}\right)^{m}\left|\partial^{\beta} \varphi(x)\right|<\infty$, e como isso vale para todo $\beta$ com $|\beta| \leq m$, segue o resultado.

É simples de se notar que:

1. Se $\varphi \in \mathcal{S}$, então para todo $\gamma \in \mathbb{N}^{n}$, a função dada por $g(x)=x^{\gamma} \varphi(x)$ é elemento de $\mathcal{S}$.

2. $\mathcal{S}$ é um subespaço vetorial de $\mathcal{C}^{\infty}\left(\mathbb{R}^{n}\right)$.

Dada uma família de semi-normas definidas em $\mathcal{S}$, existe uma topologia na qual todas as seminormas da família dada são funções contínuas. Além disso, munido dessa topologia $\mathcal{S}$ é um espaço vetorial topológico localmente convexo, metrizável e completo, ou seja, um espaço de Fréchet. Se nossa família é dada por $\mathcal{F}=\left\{F_{i}\right\}_{i \in I}$, uma base local para essa topologia é dada pelos conjuntos $A_{\varepsilon, L}=\left\{f \in \mathcal{S}: F_{i}(f)<\varepsilon, \forall i \in L\right\}$, onde $L \subseteq I$ é finito e $\varepsilon>0$. Vejamos como se relacionam algumas topologias definidas desse modo.

Lema 2.0.5 As seguinte famílias de semi-normas definem a mesma topologia em $\mathcal{S}$.

1. $\left\{\left\|x^{\alpha} \partial^{\beta} \varphi(x)\right\|_{\infty}\right\}_{\alpha, \beta \in \mathbb{N}^{n}}$.

2. $\left\{\left\|(1+|x|)^{m} \partial^{\gamma} \varphi(x)\right\|_{\infty}\right\}_{m \in \mathbb{N}, \gamma \in \mathbb{N}^{n}}$.

3. $\left\{\left\|\left(1+|x|^{2}\right)^{m} \partial^{\gamma} \varphi(x)\right\|_{\infty}\right\}_{m \in \mathbb{N}, \gamma \in \mathbb{N}^{n}}$.

4. $\left\{\left\|(1+|x|)^{m} \sup _{|\alpha| \leq p}\left|\partial^{\alpha} \varphi(x)\right|\right\|_{\infty}\right\}_{m, p \in \mathbb{N}}$.

Demonstração: Vamos comparar 1 e 2. Dados $m \in \mathbb{N}, \gamma \in \mathbb{N}^{n}$,

$$
\sup _{x \in \mathbb{R}^{n}}\left((1+|x|)^{m}\left|\partial^{\gamma} \varphi(x)\right|\right) \leq \sum_{k=0}^{m}\left(\begin{array}{c}
m \\
k
\end{array}\right) \sup _{x \in \mathbb{R}^{n}}\left(|x|^{k}\left|\partial^{\gamma} \varphi(x)\right|\right) .
$$


Temos que $|x|=\sqrt{x_{1}^{2}+\ldots+x_{n}^{2}} \leq\left(\left|x_{1}\right|+\ldots+\left|x_{n}\right|\right)$, donde $|x|^{k} \leq\left(\left|x_{1}\right|+\ldots+\left|x_{n}\right|\right)^{k}$, que é uma soma de termos do tipo $b\left|x^{\alpha}\right|$, onde $b$ é uma constante positiva, $\alpha \in \mathbb{N}^{n}$ e $|\alpha|=k$. Assim existem $s \in \mathbb{N}, \alpha_{1}, \ldots \alpha_{s} \in \mathbb{N}^{n}$ e uma constante $K>0$ independente de $\varphi$ tais que

$$
\sup _{x \in \mathbb{R}^{n}}(1+|x|)^{m}\left|\partial^{\gamma} \varphi(x)\right| \leq K \sum_{j=1}^{s} \sup _{x \in \mathbb{R}^{n}}\left|x^{\alpha_{j}} \partial^{\gamma} \varphi(x)\right|
$$

e podemos concluir que a topologia gerada por 1 é mais fina que a gerada por 2 . Dados $\alpha, \beta \in \mathbb{N}^{n}$,

$$
\sup _{x \in \mathbb{R}^{n}}\left|x^{\alpha} \partial^{\beta} \varphi(x)\right| \leq \sup _{x \in \mathbb{R}^{n}}|x|^{|\alpha|}\left|\partial^{\beta} \varphi(x)\right| \leq \sup _{x \in \mathbb{R}^{n}}(1+|x|)^{|\alpha|}\left|\partial^{\beta} \varphi(x)\right|,
$$

donde concluimos que as topologias geradas por 1 e 2 são iguais.

Comparemos agora 1 e 3 . O argumento para mostrar que a topologia gerada por 1 é mais fina que a gerada por 3 é análogo ao argumento feito para 1 e 2 . Dados $\alpha, \beta \in \mathbb{N}^{n}$,

$$
\begin{aligned}
\sup _{x \in \mathbb{R}^{n}}\left|x^{\alpha} \partial^{\beta} \varphi(x)\right| & \leq \sup _{x \in \mathbb{R}^{n}}(1+|x|)^{|\alpha|}\left|\partial^{\beta} \varphi(x)\right| \\
& =\sup _{x \in \mathbb{R}^{n}}\left(1+|x|^{2}\right)^{\frac{|\alpha|}{2}} \frac{(1+|x|)^{|\alpha|}}{\left(1+|x|^{2}\right)^{\frac{|\alpha|}{2}}}\left|\partial^{\beta} \varphi(x)\right| \\
& \leq \sup _{x \in \mathbb{R}^{n}}\left(1+|x|^{2}\right)^{|\alpha|} \frac{(1+|x|)^{|\alpha|}}{\left(1+|x|^{2}\right)^{\frac{|\alpha|}{2}}}\left|\partial^{\beta} \varphi(x)\right| .
\end{aligned}
$$

Como existe $K>0$ tal que $(1+|x|)^{|\alpha|} /\left(1+|x|^{2}\right)^{\frac{|\alpha|}{2}} \leq K, \forall x \in \mathbb{R}^{n}$, segue que as topologias geradas por 1 e 3 são iguais. Quanto à 1 e 4 , dados $m, p \in \mathbb{N}$,

$$
\begin{aligned}
\sup _{x \in \mathbb{R}^{n}}\left((1+|x|)^{m} \sup _{|\alpha| \leq p}\left|\partial^{\alpha} \varphi(x)\right|\right) & \leq \sup _{x \in \mathbb{R}^{n}}\left((1+|x|)^{m} \sum_{|\alpha| \leq p}\left|\partial^{\alpha} \varphi(x)\right|\right) \\
& \leq \sum_{|\alpha| \leq m} \sup _{x \in \mathbb{R}^{n}}\left((1+|x|)^{m}\left|\partial^{\alpha} \varphi(x)\right|\right) .
\end{aligned}
$$

Pelo que já vimos com relação à família 2 , a topologia gerada por 1 é mais fina que a gerada por 4. Da mesma forma, dados $\alpha, \beta \in \mathbb{N}^{n}$, se $p=|\beta|, m=|\alpha|$,

$$
\begin{aligned}
\sup _{x \in \mathbb{R}^{n}}\left|x^{\alpha} \partial^{\beta} \varphi(x)\right| & \leq \sup _{x \in \mathbb{R}^{n}}|x|^{|\alpha|}\left|\partial^{\beta} \varphi(x)\right| \\
& \leq \sup _{x \in \mathbb{R}^{n}}(1+|x|)^{|\alpha|}\left|\partial^{\beta} \varphi(x)\right| \\
& \leq \sup _{x \in \mathbb{R}^{n}}\left((1+|x|)^{m} \sup _{|\gamma| \leq p}\left|\partial^{\gamma} \varphi(x)\right|\right) .
\end{aligned}
$$

Conseqüentemente as topologias geradas pelas famílias 1 e 4 são iguais. 


\section{Capítulo 3}

\section{Teorema de Seidenberg-Tarski}

Definição 3.0.2 Um conjunto $A \subseteq \mathbb{R}^{n}$ é dito semi-algébrico se ele puder ser escrito como uma reunião finita de intersecções finitas de conjuntos do tipo:

$$
\left\{x \in \mathbb{R}^{n}: P(x)=0\right\},\left\{x \in \mathbb{R}^{n}: P(x)>0\right\},
$$

onde $P$ é um polinômio. Isto é, conjuntos semi-algébricos são reuniões finitas de conjuntos do tipo

$$
\left\{x \in \mathbb{R}^{n}: P_{1}(x)=0=\ldots=P_{l}(x), Q_{1}(x)>0, \ldots, Q_{r}(x)>0\right\},
$$

onde cada $P_{i}$ e cada $Q_{j}$ é um polinômio.

Lema 3.0.6 Valem as seguintes propriedades:

1. A reunião finita de conjuntos semi-algébricos é um conjunto semi-algébrico.

2. A interseç̧ão finita de conjuntos semi-algébricos é um conjunto semi-algébrico.

3. O complementar de um conjunto semi-algébrico é um conjunto semi-algébrico.

Demonstração: Vejamos a prova.

1. É suficiente mostrar que a reunião de dois conjuntos semi-algébricos é um conjunto semialgébrico, o que decorre da definição.

2. Vejamos que se $A, B$ são semi-algébricos, então $A \cap B$ também o é. De fato, digamos que $A=\cup_{i=1}^{p} A_{i}, B=\cup_{j=1}^{q} B_{j}$, onde $A_{i}, B_{j}$ são intersecções finitas de conjuntos como os de (3.0.1). Então

$$
A \cap B=\cup_{i, j}\left(A_{i} \cap B_{j}\right) .
$$

Como cada $A_{i} \cap B_{j}$ pode ser escrito como uma intersecção finita de conjuntos como os de (3.0.1), $A \cap B$ é semi-algébrico pelo item 1 .

3. Seja $A$ semi-algébrico. Escrevendo $A=\cup_{k=1}^{l} A_{k}$, onde $A_{k}=\cap_{i=1}^{n_{k}} B_{i}^{k}$, e cada $B_{i}^{k}$ é como algum dos conjuntos de (3.0.1), então

$$
\begin{aligned}
A^{c} & =\cap_{k=1}^{l} A_{k}{ }^{c} \\
& =\cap_{k=1}^{l}\left(\cup_{i=1}^{n_{k}}\left(B_{i}^{k}\right)^{c}\right)
\end{aligned}
$$

Pelos itens 1 e 2 é suficiente notar que cada $\left(B_{i}^{k}\right)^{c}$ é semi-algébrico. 
Agora enunciaremos e provaremos um dos teoremais centrais desse trabalho.

Teorema 3.0.5 (Seidenberg-Tarski) $S e A \subseteq \mathbb{R}^{n+m}$ é semi-algébrico, então a projeção de $A$ em $\mathbb{R}^{m}$ também é um conjunto semi-algébrico.

Provaremos inicialmente o teorema 3.0.5 para $n=1$. Vamos denotar as variáveis em $\mathbb{R}$ por $x$ e em $\mathbb{R}^{m}$ por $y$. Queremos provar que

$$
\left\{y \in \mathbb{R}^{m}: \exists x \in \mathbb{R}:(x, y) \in A\right\}
$$

é um conjunto semi-algébrico, se $A \subseteq \mathbb{R}^{1+m}$ é semi-algébrico. Como a imagem de uma reunião por uma função é a reunião das imagens, podemos supor, sem perda de generalidade, que $A$ é descrito por

$$
P_{1}(x, y)>0, \ldots, P_{s}(x, y)=0,
$$

onde os $P_{i}$ são polinômios. Provaremos o teorema 3.0 .5 por indução no grau máximo dos polinômios que aparecem em (3.0.2) com relação à $x$.

Definição 3.0.3 Dado $x \in \mathbb{R}$, vamos definir o sinal de $x$ como:

$$
\operatorname{sgn}(x)=\left\{\begin{array}{r}
0, \text { se } x=0 \\
1, \text { se } x>0 \\
-1, \text { se } x<0
\end{array}\right.
$$

Começaremos fazendo algumas observações sobre polinômios em $\mathbb{R}$. Sejam $Q_{1}, \ldots Q_{s}$ polinômios em uma variável real de grau no máximo $k$, não identicamente nulos. Sejam

$$
x_{1}<x_{2}<\ldots<x_{N}
$$

os pontos de $\mathbb{R}$ onde ao menos um dos polinômios $Q_{1}, \ldots, Q_{s}$ se anula. Digamos que $x_{0}=-\infty$, $x_{N+1}=\infty$ e $\left.I_{k}=\right] x_{k}, x_{k+1}\left[, k=0, \ldots, N\right.$. Dados $1 \leq i \leq s, 0 \leq k \leq N, \operatorname{sgn} Q_{i}(x)$ independe de $x$ quando $x \in I_{k}$. Escreveremos então

$$
\operatorname{sgn} Q_{i}\left(I_{k}\right)=\operatorname{sgn} Q_{i}(x), \forall x \in I_{k} .
$$

Vamos resumir todas as informações sobre zeros e mudanças de sinal em

$$
S G N\left(Q_{1}, \ldots, Q_{s}\right)=\left\{\operatorname{sgn} Q_{i}\left(x_{j}\right), \operatorname{sgn} Q_{i}\left(I_{k}\right), i=1, \ldots, s ; j=1, \ldots N ; k=0, \ldots N\right\}
$$

Mais especificamente,

$$
\begin{aligned}
& S G N\left(Q_{1}, \ldots Q_{s}\right)=\left\{\operatorname{sgn} Q_{1}\left(x_{1}\right), \operatorname{sgn} Q_{1}\left(I_{0}\right), \ldots, \operatorname{sgn} Q_{s}\left(x_{1}\right), \operatorname{sgn} Q_{s}\left(I_{0}\right), \operatorname{sgn} Q_{1}\left(x_{2}\right),\right. \\
& \left.\operatorname{sgn} Q_{1}\left(I_{1}\right), \ldots, \operatorname{sgn} Q_{s}\left(x_{N}\right), \operatorname{sgn} Q_{s}\left(I_{N-1}\right), \operatorname{sgn} Q_{1}\left(I_{N}\right), \ldots, \operatorname{sgn} Q_{s}\left(I_{N}\right)\right\}
\end{aligned}
$$

Esse é um elemento da reunião disjunta

$$
W=\cup_{p=0}^{k s}\{-1,0,1\}^{s(2 p+1)},
$$


onde $k s$ é o número máximo de raízes (que ocorre se todos os polinômios tiverem grau $k$ e raízes distintas, todas reais.) Vale notar que nem todo elemento $w \in W$ pode ocorrer, pois se, por exemplo, $\operatorname{sgn} Q_{i}\left(x_{j}\right) \neq 0$, então devemos ter que

$$
\operatorname{sgn} Q_{i}\left(x_{j}\right)=\operatorname{sgn} Q_{i}\left(I_{j-1}\right)=\operatorname{sgn} Q_{i}\left(I_{j}\right) .
$$

Conhecendo $S G N\left(Q_{1}, \ldots Q_{s}\right)$ podemos decidir se algum sistema do tipo

$$
Q_{1}(x)>0, \ldots, Q_{s}(x)=0
$$

é satisfeito para algum $x \in \mathbb{R}$. De fato, se algum polinômio $Q_{i}$ aparece em (3.0.3) como $Q_{i}(x)=0$, então (3.0.3) é equivalente a

$$
0 \in\left\{\operatorname{sgn} Q_{1}\left(x_{1}\right), \ldots, \operatorname{sgn} Q_{s}\left(x_{1}\right), \ldots, \operatorname{sgn} Q_{1}\left(x_{N}\right), \ldots, \operatorname{sgn} Q_{s}\left(x_{N}\right)\right\}
$$

Se, do contrário, todos os polinômios $Q_{j}$ em (3.0.3) aparecem como $Q_{j}(x)>0,(3.0 .3)$ é equivalente a existir existir $k$ tal que $0 \leq k \leq N$ e

$$
\operatorname{sgn} Q_{1}\left(I_{k}\right)=1, \ldots \operatorname{sgn} Q_{s}\left(I_{k}\right)=1 .
$$

Tendo em vista as observações feitas acima, o seguinte resultado implicará o teorema 3.0.5 no caso em que $n=1$ e o grau máximo dos polinômios $P_{1}, \ldots, P_{s}$ com relação a $x$ é $k$.

Teorema 3.0.6 Sejam $P_{1}(x, y), \ldots, P_{s}(x, y)$ polinômios. Então

$$
E=\left\{y \in \mathbb{R}^{m}: S G N\left(P_{1}(., y), \ldots, P_{s}(., y)\right)=w\right\}
$$

é semi-algébrico, para todo $w \in W$.

O seguinte lema nos auxiliará a provar o teorema 3.0.6 por indução em $k$, onde $k$ é o grau máximo dos polinômios $P_{1}, \ldots, P_{s}$ com relação a $x$.

Lema 3.0.7 Sejam $P_{1}, \ldots, P_{s}$ polinômios reais de grau no máximo $k>0$ tais que $P_{s}$ tem grau exatamente $k$ e nenhum deles é identicamente nulo. Se $g_{1}, \ldots, g_{s}$ são os restos obtidos quando dividimos $P_{s}$ por $P_{1}, \ldots, P_{s-1}, P_{s}^{\prime}$, então

$$
\operatorname{SGN}\left(P_{1}, \ldots, P_{s-1}, P_{s}^{\prime}, g_{1}, \ldots, g_{s}\right)
$$

determina $S G N\left(P_{1}, \ldots, P_{s}\right)$.

Demonstração: Sejam $x_{1}<\ldots<x_{N}$ os pontos de $\mathbb{R}$ onde algum dos polinômios

$$
P_{1}, \ldots, P_{s-1}, P_{s}^{\prime}, g_{1}, \ldots, g_{s}
$$

se anula (desconsideraremos os polinômios identicamente nulos). Conhecer

$$
\operatorname{SGN}\left(P_{1}, \ldots, P_{s-1}, P_{s}^{\prime}, g_{1}, \ldots, g_{s}\right)
$$

nos permite concluir quais desses pontos, digamos

$$
x_{i_{1}}<\ldots<x_{i_{l}},
$$


são zeros de $P_{1}, \ldots, P_{s}, P_{s}^{\prime}$ (mesmo sem saber quais são, de fato, esses pontos). Como $P_{s}(x)=$ $g_{j}(x)$ se $P_{j}(x)=0$, para $1 \leq j \leq s-1$, e $P_{s}(x)=g_{s}(x)$ se $P_{s}^{\prime}(x)=0$, podemos determinar $\operatorname{sgn} P_{s}\left(x_{i_{j}}\right)$.

Observe que o sinal do coeficiente dominante de $P_{s}$ é igual à $\operatorname{sgn} P_{s}^{\prime}\left(I_{N}\right)\left(\operatorname{se} \operatorname{sgn} P_{s}^{\prime}\left(I_{N}\right)=0\right.$, então $P_{s}$ é constante, e portanto não tem zeros).

Nos intervalos ] $-\infty, x_{i_{1}}[, \ldots,] x_{i_{l}}, \infty$ [ o polinômio $P_{s}$ é monótono (uma vez que as raízes de $P_{s}^{\prime}$ são elementos de $\left\{x_{i_{1}}, \ldots, x_{i_{l}}\right\}$ ). Assim sendo, $P_{s}$ tem um zero em um desses intervalos se, e somente se, $P_{s}$ tem sinais opostos nos extremos (onde os sinais em $\infty$ e em $-\infty$ são dados pelo sinal do coeficiente dominante de $P_{s}$ ).

Dessa forma podemos checar quais desses intervalos contém um zero de $P_{s}$ (note que se houver, há um único zero). Esses zeros, juntamente com os pontos $x_{i_{1}}, \ldots, x_{i_{l}}$ que não são somente zeros de $P_{s}^{\prime}$ nos fornecem todos os zeros relevantes de $P_{1}, \ldots, P_{s}$; considerando também as informações sobre $\operatorname{sgn} P_{s}\left(x_{i_{j}}\right)$ e o fato de que conhecemos os sinais dos $P_{i}$ nos intervalos que contém os zeros de $P$, podemos obter $S G N\left(P_{1}, \ldots, P_{s}\right)$.

Observe que quando substituimos $P_{1}, \ldots P_{s}$ por $P_{1}, \ldots, P_{s-1}, P_{s}^{\prime}, g_{1}, \ldots, g_{s}$ estamos aumentando o número de polinômios, mas diminuindo o número de polinômios cujos graus são exatamente $k$.

Demonstração: (do teorema 3.0.6) Para isso faremos uma indução dupla: assumiremos que o resultado é válido para todo natural menor do que $k$ e também para $k$, desde que o número de polinômios cujo grau com relação a $x$ é $k$ seja menor.

Sejam $P_{1}(x, y), \ldots, P_{s}(x, y)$ polinômios de grau no máximo $k$ em $x$.

Fixando $m_{1}, \ldots, m_{s} \in \mathbb{N}$ entre 0 e $k$, o seguinte conjunto é semi-algébrico:

$$
\left\{y \in \mathbb{R}^{m} \text { tal que } P_{1}(., y) \text { tem grau } m_{1}, \ldots, P_{s}(., y) \text { tem grau } m_{s}\right\}
$$

De fato, podemos escrever que

$$
P_{i}(x, y)=Q_{0}^{i}(y)+x Q_{1}^{i}(y)+\ldots+x^{m_{i}} Q_{m_{i}}^{i}(y)+\ldots+x^{k} Q_{k}^{i}(y),
$$

onde todos os $Q_{j}^{i}(y)$ são polinômios em $y$. Assim, o grau de $P_{i}(., y)$ é $m_{i}$ se, e somente se, $Q_{m_{i}}^{i}(y) \neq 0$ e $Q_{j}^{i}(y)=0, \forall j>m_{i}$.

Como

$$
\mathbb{R}^{m}=\bigcup_{0 \leq c_{l} \leq k}\left\{y \in \mathbb{R}^{m}: \text { grau de } P_{j}(., y) \text { é } c_{j}, \forall 1 \leq j \leq s\right\}
$$

e $E=E \cap \mathbb{R}^{m}$, então basta provar que se $0 \leq c_{1}, \ldots, c_{s} \leq k$, então

$$
E \cap\left\{y \in \mathbb{R}^{m}: P_{j}(., y) \text { tem grau } c_{j}, \forall 1 \leq j \leq s\right\}
$$

é semi-algébrico. Sejam $m_{1}, \ldots m_{s}$ entre 0 e $k$. Seja

$$
B=\left\{y \in \mathbb{R}^{m}: P_{j}(., y) \text { tem grau } m_{j}, \forall 1 \leq j \leq s\right\} .
$$

Então

$$
B=\left\{y \in \mathbb{R}^{m}: Q_{m_{1}}^{1}(y) \ldots Q_{m_{s}}^{s}(y) \neq 0\right\} \cap\left(\cap_{i=1}^{s}\left\{y \in \mathbb{R}^{m}: Q_{j}^{i}(y)=0, \forall j>m_{j}\right\}\right),
$$


donde é suficiente provarmos que

$$
E \cap\left\{y \in \mathbb{R}^{m}: Q_{m_{1}}^{1}(y) \ldots Q_{m_{s}}^{s}(y) \neq 0\right\}
$$

é um conjunto semi-algébrico.

Alterando a ordem dos $P_{j}$, se necessário, podemos assumir que $m_{s}=\max \left\{m_{i}: 1 \leq i \leq s\right\}$.

Vamos considerar os polinômios $P_{i}$ como polinômios em $x$. Dividindo formalmente $P_{s}$ por $P_{1}, \ldots, P_{s-1}, P_{s}^{\prime}$ teremos

$$
\begin{gathered}
P_{s}(x, y)=P_{j}(x, y) \cdot q_{j}(x, y)+r_{j}(x, y), \forall j \leq s-1 ; \\
P_{s}(x, y)=P_{s}^{\prime}(x, y) \cdot q_{s}(x, y)+r_{s}(x, y) .
\end{gathered}
$$

Multiplicando por $\left(Q_{m_{j}}^{j}(y)\right)^{2}$ teremos novamente expressões bem definidas. Podemos escrever

$$
\begin{gathered}
\left(Q_{m_{j}}^{j}(y)\right)^{2} P_{s}(x, y)=P_{j}(x, y) \cdot q_{j}^{*}(x, y)+\left(Q_{m_{j}}^{j}(y)\right)^{2} r_{j}(x, y), \forall j \leq s-1 \\
\left(Q_{m_{s}}^{s}(y)\right)^{2} P_{s}(x, y)=P_{s}^{\prime}(x, y) \cdot q_{s}^{*}(x, y)+\left(Q_{m_{s}}^{s}(y)\right)^{2} r_{s}(x, y)
\end{gathered}
$$

Sejam $g_{j}(x, y)=\left(Q_{m_{j}}^{j}(y)\right)^{2} r_{j}(x, y)$. Assim sendo,

$$
\begin{gathered}
\left\{y \in E: Q_{m_{1}}^{1}(y) \ldots Q_{m_{s}}^{s}(y) \neq 0\right\}=\left\{y \in \mathbb{R}^{m}: S G N\left(P_{1}(., y), \ldots, P_{s}(., y)\right)=w \mathrm{e}\right. \\
\left.Q_{m_{1}}^{1}(y) \ldots Q_{m_{s}}^{s}(y) \neq 0\right\}
\end{gathered}
$$

se escreve, pelo lema 3.0.7, como uma reunião finita de conjuntos da forma

$$
\begin{aligned}
& \left\{y \in \mathbb{R}^{m}: S G N\left(\frac{P_{1}(., y)}{Q_{m_{1}}^{1}(y)^{2}}, \ldots, \frac{P_{s-1}(., y)}{Q_{m_{s-1}}^{s-1}(y)^{2}}, \frac{P_{s}^{\prime}(., y)}{Q_{m_{s}}^{s}(y)^{2}}, \frac{g_{1}(., y)}{\left(Q_{m_{1}}^{1}(y)\right)^{2}}, \ldots, \frac{g_{s}(y)}{\left(Q_{m_{s}}^{s}(y)\right)^{2}}\right)=w^{\prime} \mathrm{e}\right. \\
& \left.Q_{m_{1}}^{1}(y) \ldots Q_{m_{s}}^{s}(y) \neq 0\right\}
\end{aligned}
$$

Como $\operatorname{sgn}\left(Q_{m_{j}}^{j}(y)\right)^{2}=1$, então $E \cap B$ se escreve como uma reunião finita de conjuntos do tipo

$$
\begin{aligned}
& \left\{y \in \mathbb{R}^{m}: S G N\left(P_{1}(., y), \ldots, P_{s-1}(., y), P_{s}^{\prime}(., y), g_{1}(., y), \ldots, g_{s}(., y)\right)=w^{\prime} \mathrm{e}\right. \\
& \left.Q_{m_{1}}^{1}(y) \ldots Q_{m_{s}}^{s}(y) \neq 0\right\}
\end{aligned}
$$

Pela Hipótese de Indução concluimos a prova.

Observação Com essa prova provamos o teorema 3.0.5 no caso em que $E \subseteq \mathbb{R}^{1+m}$ é semialgébrico. Resta ver para $n$ qualquer. Vejamos como fazer se $n=2$, já que o mesmo argumento nos possibilita provar por indução o caso geral. Seja $E \subseteq \mathbb{R}^{2+m}$ um conjunto semi-algébrico. Pelo que acabamos de provar,

$$
A=\left\{(y, z) \in \mathbb{R}^{1+m}: \exists x \in \mathbb{R}:(x, y, z) \in E\right\}
$$

é semi-algébrico. Mas então aplicando novamente o teorema 3.0.5 para o caso $n=1$ temos que

$$
B=\left\{z \in \mathbb{R}^{m}: \exists y \in \mathbb{R}:(y, z) \in A\right\}=\left\{z \in \mathbb{R}^{m}: \exists(x, y) \in \mathbb{R}^{2}:(x, y, z) \in E\right\}
$$

é semi-algébrico. 
Corolário 3.0.1 Seja $E \subseteq \mathbb{R}^{n+m}$ semi-algébrico. Então

$$
\left\{y \in \mathbb{R}^{m}: \forall x \in \mathbb{R}^{n},(x, y) \notin E\right\}
$$

é semi-algébrico.

Demonstração: De fato,

$$
\left\{y \in \mathbb{R}^{m}: \forall x \in \mathbb{R}^{n},(x, y) \notin E\right\}=\left\{y \in \mathbb{R}^{m}: \exists x \in \mathbb{R}^{n}:(x, y) \in E\right\}^{c} .
$$

Corolário 3.0.2 Se $E \subseteq \mathbb{R}^{n+m}$ é semi-algébrico, então o conjunto

$$
\left\{y \in \mathbb{R}^{m}: \forall x \in \mathbb{R}^{n},(x, y) \in E\right\}
$$

é semi-algébrico.

Demonstração: De fato,

$$
\left\{y \in \mathbb{R}^{m}: \forall x \in \mathbb{R}^{n},(x, y) \in E\right\}=\left\{y \in \mathbb{R}^{m}: \exists x \in \mathbb{R}^{n}:(x, y) \in E^{c}\right\}^{c} .
$$

Definição 3.0.4 Seja $E \subseteq \mathbb{R}^{n}$. Uma função $g: E \rightarrow \mathbb{R}$ é dita semi-algébrica se o seu subgráfico for semi-algébrico, isto é, se o conjunto

$$
F=\{(x, y) \in E \times \mathbb{R}: y \leq g(x)\}
$$

for semi-algébrico.

Corolário 3.0.3 Seja $E \subseteq \mathbb{R}^{2+n}$ um conjunto semi-algébrico. Vamos denotar as coordenadas de $\mathbb{R}^{2+n}$ por $(x, y, z)$, onde $x, y \in \mathbb{R}, z \in \mathbb{R}^{n}$. Seja

$$
f(x)=\sup \left\{y \in \mathbb{R}: \exists z \in \mathbb{R}^{n}:(x, y, z) \in E\right\},
$$

com a convenção de que $f(x)=-\infty$ se não existirem $y, z$ tais que $(x, y, z) \in$ E. Então $f$ é semi-algébrica.

Demonstração: De fato,

$$
F=\left\{(x, y) \in \mathbb{R}^{2}: \forall \varepsilon>0, \exists y^{\prime}, z: y^{\prime} \in \mathbb{R}, z \in \mathbb{R}^{n}: y-\varepsilon<y^{\prime},\left(x, y^{\prime}, z\right) \in E\right\} .
$$

Pelo teorema 3.0.5 o seguinte conjunto é semi-algébrico:

$$
A=\left\{(x, y): \exists z \in \mathbb{R}^{n}:(x, y, z) \in E\right\} .
$$

Segue-se que também é semi-algébrico o seguinte conjunto:

$$
B=\left\{\left(x, y, y^{\prime}, \varepsilon\right) \in \mathbb{R}^{4}: \varepsilon>0, y-\varepsilon<y^{\prime},\left(x, y^{\prime}\right) \in A\right\} .
$$

Assim,

$$
\begin{aligned}
F & =\left\{(x, y) \in \mathbb{R}^{2}: \forall \varepsilon>0, \exists y^{\prime} \in \mathbb{R}: y^{\prime}>y-\varepsilon,\left(x, y^{\prime}\right) \in A\right\} \\
& =\left\{(x, y) \in \mathbb{R}^{2}: \forall \varepsilon>0, \exists y^{\prime} \in \mathbb{R}:\left(x, y, y^{\prime}, \varepsilon\right) \in B\right\}
\end{aligned}
$$

é semi-algébrico. 


\section{Capítulo 4}

\section{Séries de Puiseux}

Lema 4.0.8 Seja $P(x, y)$ um polinômio em $y \in \mathbb{R}$ da forma

$$
P(x, y)=y^{n}+B_{1}(x) y^{n-1}+\ldots+B_{n}(x),
$$

onde cada $B_{j}$ é um polinômio em $\mathbb{R}^{N}$. Vamos assumir que $P(0, y)$ se escreve como o produto de dois fatores relativamente primos de graus $p$ e q, isto é,

$$
P(0, y)=g_{0}(y) h_{0}(y)
$$

onde $g_{0}, h_{0}$ são polinômios reais que não tem fatores comuns.

Então $P(x, y)=G(x, y) \cdot H(x, y)$, onde $G(x, y)$ e $H(x, y)$ são polinômios em y cujos respectivos graus são $p$ e q e cujos coeficientes são polinômios em $x$. Além disso,

$$
G(0, y)=g_{0}(y), H(0, y)=h_{0}(y)
$$

Demonstração: Podemos escrever que

$$
P(x, y)=P(0, y)+x f_{1}(y)+\ldots+x^{r} f_{r}(y),
$$

onde todos os $f_{j}$ são polinômios reais de grau menor ou igual a $n-1$. Desejamos escrever

$$
\begin{aligned}
& G(x, y)=g_{0}(y)+x g_{1}(y)+\ldots+x^{r} g_{r}(y), \\
& H(x, y)=h_{0}(y)+x h_{1}(y)+\ldots+x^{r} h_{r}(y),
\end{aligned}
$$

onde $g_{1}(y), \ldots, g_{r}(y)$ são polinômios de grau no máximo $p-1$, enquanto $h_{1}(y), \ldots, h_{r}(y)$ são polinômios de grau no máximo $q-1$. Multiplicando as expressões de $G$ e de $H$, vemos que as seguintes equações devem ser satisfeitas:

$$
\begin{aligned}
& P(0, y)=g_{0}(y) h_{0}(y), \\
& f_{1}(y)=g_{0}(y) h_{1}(y)+g_{1}(y) h_{0}(y), \\
& \ldots \\
& f_{r}(y)=g_{0}(y) h_{r}(y)+\ldots+g_{r} h_{0}(y) .
\end{aligned}
$$

Provaremos por indução que existem funções $g_{1}, \ldots, g_{r}, h_{1}, \ldots, h_{r}$ que satisfazem essas equações. A primeira equação é verificada por hipótese. 
Suponhamos que existam funções $g_{1}, \ldots, g_{l-1}, h_{1}, \ldots, h_{l-1}$ que satisfazem as $l$ primeiras equações. Existe um polinômio $C_{l}$, de grau menor ou igual a $n-1$, tal que a equação que $g_{l}$ e $h_{l}$ devem verificar pode ser escrita como

$$
g_{0}(y) h_{l}(y)+h_{0}(y) g_{l}(y)=C_{l}(y)
$$

Como $g_{0}$ e $h_{0}$ são relativamente primos, então existem polinômios $g_{l}, h_{l}$ cujos graus não ultrapassam $p-1$ e $q-1$, respectivamente, tais que a equação acima é satisfeita.

Teorema 4.0.7 (Lema de Hensel) Seja $P(x, y)$ um polinômio em $y \in \mathbb{R}$ da forma

$$
P(x, y)=y^{n}+B_{1}(x) y^{n-1}+\ldots+B_{n}(x),
$$

onde cada $B_{i}$ é uma função real analítica em uma vizinhança da origem em $\mathbb{R}^{N}$. Se

$$
P(0, y)=g_{0}(y) h_{0}(y)
$$

onde $g_{0}$ e $h_{0}$ são polinômios reais sem fatores comuns cujos graus são $p$ e $q(\operatorname{com} p+q=n)$, então podemos escrever, para $x$ pequeno,

$$
P(x, y)=G(x, y) \cdot H(x, y),
$$

onde $G(x, y)$ e $H(x, y)$ são polinômios em y de graus p e q cujos coeficientes são funções analíticas reais para $x$ pequeno. Além disso,

$$
G(0, y)=g_{0}(y), H(0, y)=h_{0}(y) .
$$

Demonstração: Seja $E(k)$ o conjunto dos polinômios mônicos de grau $k$ com coeficientes reais. Podemos supor que $P(0, y)=g_{0} h_{0}$, onde $g_{0} \in E(p), h_{0} \in E(q)$. Seja

$$
\Lambda: \mathbb{R} \times E(p) \times E(q) \longrightarrow E(p+q)
$$

dada por $\Lambda(x, R, S)=P(x,)-.R . S$. Temos, por hipótese, que $\Lambda\left(0, g_{0}, h_{0}\right)=0$. Queremos provar que podemos resolver a equação

$$
\Lambda(x, R(x), S(x))=0, R(0)=g_{0}, S(0)=h_{0} .
$$

Como os espaços $E(n)$ não são espaços vetoriais, não podemos simplesmente utilizar o Teorema da Função Implícita. Denotaremos por $\mathbb{R}_{m}[y]$ o espaço dos polinômios de grau menor ou igual a $\mathrm{m}$ com coeficientes reais. A função

$$
\pi_{m}: E(m) \longrightarrow \mathbb{R}_{m-1}[y]
$$

dada por $\pi_{m}(R)=R-y^{m}$ é uma bijeção. Além disso, dados $R \in E(p), S \in E(q)$,

$$
\pi_{p+q}(R . S)=\pi_{p}(R) \cdot \pi_{q}(S)+y^{q} \pi_{p}(R)+y^{p} \pi_{q}(S) .
$$

De fato, se

$$
\begin{gathered}
R=a_{0}+\ldots+a_{p-1} y^{p-1}+y^{p} \\
S=b_{0}+\ldots+b_{q-1} y^{q-1}+y^{q}
\end{gathered}
$$


$\pi_{p}(R)=a_{0}+\ldots+a_{p-1} y^{p-1}, \pi_{q}(S)=b_{0}+\ldots+b_{q-1} y^{q-1}$, donde $\pi_{p}(R) \cdot \pi_{q}(S)=a_{0} b_{0}+\left(a_{0} b_{1}+\right.$ $\left.b_{0} a_{1}\right) y+\ldots+a_{p-1} b_{q-1} y^{p+q-2}$. Além disso,

$$
\begin{aligned}
\pi_{p+q}(R . S) & =a_{0} b_{0}+\left(a_{0} b_{1}+a_{1} b_{0}\right) y+\ldots\left(a_{p-1}+b_{q-1}\right) y^{q+p-1} \\
& =a_{0} b_{0}+\left(a_{0} b_{1}+a_{1} b_{0}\right) y+\ldots+a_{p-1} b_{q-1} y^{p+q-2}+ \\
& +y^{q}\left(a_{0}+\ldots+a_{p-1} y^{p-1}\right)+y^{p}\left(b_{0}+\ldots+b_{q-1} y^{q-1}\right) .
\end{aligned}
$$

Consideremos agora

$$
\Gamma: \mathbb{R} \times \mathbb{R}_{p-1}[y] \times \mathbb{R}_{q-1}[y] \longrightarrow \mathbb{R}_{p+q-1}[y]
$$

dada por

$$
\Gamma(x, g, h)=\pi_{p+q}(P(x, .))-g h-y^{q} g-y^{p} h .
$$

Temos que $\Gamma\left(0, \pi_{p}\left(g_{0}\right), \pi_{q}\left(h_{0}\right)\right)=0$ por $(4.0 .1)$, pois

$$
\begin{aligned}
\Gamma\left(0, \pi_{p}\left(g_{0}\right), \pi_{q}\left(h_{0}\right)\right) & =\pi_{p+q}(P(0, .))-\pi_{p}\left(g_{0}\right) \pi_{q}\left(h_{0}\right)-y^{q} \pi_{p}\left(g_{0}\right)-y^{p} \pi_{q}\left(h_{0}\right) \\
& =\pi_{p+q}\left(g_{0} h_{0}\right)-\pi_{p}\left(g_{0}\right) \pi_{q}\left(h_{0}\right)-y^{q} \pi_{p}\left(g_{0}\right)-y^{p} \pi_{q}\left(h_{0}\right) \\
& =0 .
\end{aligned}
$$

Pelo Teorema da Função Implícita, basta provarmos que a derivada de $\Gamma$ com relação a $(g, h)$ no ponto $\left(0, \pi_{p}\left(g_{0}\right), \pi_{q}\left(h_{0}\right)\right)$ é um isomorfismo entre os espaços vetoriais $\mathbb{R}_{p-1}[y] \times \mathbb{R}_{q-1}[y]$ e $\mathbb{R}_{p+q-1}[y]$.

$\operatorname{Dados}(g, h) \in \mathbb{R}_{p-1}[y] \times \mathbb{R}_{q-1}[y]$ e $t \in \mathbb{R}$,

$$
\begin{aligned}
& \Gamma\left(0, \pi_{p}\left(g_{0}\right)+t g, \pi_{q}\left(h_{0}\right)+t h\right)-\Gamma\left(0, \pi_{p}\left(g_{0}\right), \pi_{q}\left(h_{0}\right)\right) \\
& \quad=\pi_{p+q}(P(0, .))-\left(\pi_{p}\left(g_{0}\right)+t g\right)\left(\pi_{q}\left(h_{0}\right)+t h\right)-y^{q}\left(\pi_{p}\left(g_{0}\right)+t g\right)-y^{p}\left(\pi_{q}\left(h_{0}\right)+t h\right) \\
& \quad-\left[\pi_{p+q}(P(0, .))-\pi_{p}\left(g_{0}\right) \pi_{q}\left(h_{0}\right)-y^{q} \pi_{p}\left(g_{0}\right)-y^{p} \pi_{q}\left(h_{0}\right)\right] \\
& \quad=t \pi_{p}\left(g_{0}\right) h-t \pi_{q}\left(h_{0}\right) g-t^{2} g h-t y^{q} g-t y^{p} h .
\end{aligned}
$$

Dessa forma vemos que a derivada de $\Gamma$ com relação a $(g, h)$ no ponto $\left(0, \pi_{p}\left(g_{0}\right), \pi_{q}\left(h_{0}\right)\right)$ é a aplicação

$$
(g, h) \longrightarrow-\pi_{p}\left(g_{0}\right) h-\pi_{q}\left(h_{0}\right) g-y^{q} g-y^{p} h=-g_{0} h-h_{0} g .
$$

Essa aplicação tem como domínio $\mathbb{R}_{p-1}[y] \times \mathbb{R}_{q-1}[y]$, e como contradomínio $\mathbb{R}_{p+q-1}[y]$ (que têm a mesma dimensão: $p+q=n)$. Como $\operatorname{mdc}\left(g_{0}, h_{0}\right)=1$, então essa aplicação é sobrejetora, e portanto bijetora.

Teorema 4.0.8 (Decomposição) Seja $P(x, y)$ um polinômio em $y \in \mathbb{R}$ da forma

$$
P(x, y)=y^{n}+B_{1}(x) y^{n-1}+\ldots+B_{n}(x),
$$

onde cada $B_{i}$ é uma função real analítica em uma vizinhança da origem em $\mathbb{R}$. Então existe $p \in \mathbb{N}$ tal que $P$ pode ser escrito, em uma vizinhança de 0 , como

$$
P\left(x^{p}, y\right)=\left(y-R_{1}(x)\right)\left(y-R_{2}(x)\right) \ldots\left(y-R_{m}(x)\right) G(x, y),
$$

onde as funções $R_{1}, \ldots, R_{m}$ são funções analíticas em uma vizinhança de $0, G(x, y)$ é um polinômio em y que não tem raízes reais para $x \neq 0$ pequeno e cujos coeficientes são funções analíticas (em x) em uma vizinhança de 0 . 
Demonstração: Faremos a prova por indução no grau do polinômio $P$. Se o grau é $n=1$, então $P(x, y)=y-\left(-B_{1}(x)\right)$, e o resultado é imediato.

Dado $n>1$, vamos assumir que a decomposição enunciada é possível para todo polinômio como em (4.0.2) cujo grau é menor que $n$. Seja $y^{\prime}=y+\frac{B_{1}(x)}{n}$; substituindo em $P(x, y)$ obtemos um novo polinômio:

$$
\begin{aligned}
P^{\prime}\left(x, y^{\prime}\right) & =\left(y^{\prime}\right)^{n}-\left(y^{\prime}\right)^{n-1} B_{1}(x)+\sum_{j<n-1}\left(\begin{array}{c}
n \\
j
\end{array}\right)\left(y^{\prime}\right)^{j}\left(\frac{-B_{1}(x)}{n}\right)^{n-j}+ \\
& +B_{1}(x)\left(y^{\prime}\right)^{n-1}+B_{1}(x)\left(\sum_{j<n-1}\left(\begin{array}{c}
n-1 \\
j
\end{array}\right)\left(y^{\prime}\right)^{j}\left(\frac{-B_{1}(x)}{n}\right)^{n-1-j}\right)+\ldots+B_{n}(x) \\
& =\left(y^{\prime}\right)^{n}+B_{2}^{\prime}(x)\left(y^{\prime}\right)^{n-2}+\ldots+B_{n-1}^{\prime}(x) y^{\prime}+B_{n}^{\prime}(x),
\end{aligned}
$$

onde as funções $B_{2}^{\prime}, \ldots, B_{n}^{\prime}$ são analíticas em $x$. Se elas se anularem identicamente, então $P(x, y)=$ $\left(y+\frac{1}{n} B_{1}(x)\right)^{n}$, e conseguimos decompor $P$ da forma desejada.

Podemos assumir, então, que existe $j$ tal que a função $B_{j}^{\prime}$ não se anula identicamente. Para cada $j$ tal que $B_{j}^{\prime}$ não se anula identicamente, seja $p_{j}$ a menor potência de $x$ que aparece em $B_{j}$. Seja $\sigma=\min \left\{\frac{p_{j}}{j}: B_{j}^{\prime}\right.$ não é nula $\}$; vamos escrever que $\sigma=\frac{l}{k}$, onde $l, k \in \mathbb{N}$ e $\frac{l}{k}$ é irredutível. Sejam $x=\left(x^{\prime \prime}\right)^{k}, y^{\prime}=\left(x^{\prime \prime}\right)^{l} y^{\prime \prime}$. Substituindo em $P^{\prime}\left(x, y^{\prime}\right)$ obtemos um novo polinômio (aqui $\left.B_{j}^{\prime \prime}\left(x^{\prime \prime}\right)=B_{j}^{\prime}\left(\left(x^{\prime \prime}\right)^{k}\right)\right)$ :

$$
\begin{aligned}
& \left(y^{\prime \prime}\right)^{n}\left(x^{\prime \prime}\right)^{n l}+B_{2}^{\prime \prime}\left(x^{\prime \prime}\right)\left(y^{\prime \prime}\right)^{n-2}\left(x^{\prime \prime}\right)^{(n-2) l}+\ldots+y^{\prime \prime}\left(x^{\prime \prime}\right)^{l} B_{n-1}^{\prime \prime}\left(x^{\prime \prime}\right)+B_{n}^{\prime \prime}\left(x^{\prime \prime}\right) \\
& =\left(x^{\prime \prime}\right)^{n l}\left(\left(y^{\prime \prime}\right)^{n}+\left(y^{\prime \prime}\right)^{n-2}\left(x^{\prime \prime}\right)^{-2 l} B_{2}^{\prime \prime}+\ldots+\left(x^{\prime \prime}\right)^{-n l} B_{n}^{\prime \prime}\right) .
\end{aligned}
$$

Notemos que $p_{j}-j \frac{l}{k} \geq 0, \forall j$ e que existe (ao menos) um $j_{*}$ tal que $p_{j_{*}}-j_{*} \frac{l}{k}=0$. Como a menor potência de $x^{\prime \prime}$ que aparece em $B_{j}^{\prime \prime}$ é $p_{j} . k$ e $p_{j} . k \geq l . j$,

$$
P^{\prime \prime}\left(x^{\prime \prime}, y^{\prime \prime}\right)=\left(y^{\prime \prime}\right)^{n}+\left(y^{\prime \prime}\right)^{n-2}\left(x^{\prime \prime}\right)^{-2 l} B_{2}^{\prime \prime}+\ldots+y^{\prime \prime}\left(x^{\prime \prime}\right)^{(1-n) l} B_{n-1}^{\prime \prime}+\left(x^{\prime \prime}\right)^{-n l} B_{n}^{\prime \prime}
$$

é um polinômio em $y^{\prime \prime}$ cujos coeficientes são funções reais analíticas em $x^{\prime \prime}$. Consideremos as raízes de $P^{\prime \prime}\left(0, y^{\prime \prime}\right)$. Como $l \cdot j_{*}=p_{j_{*}} \cdot k$, segue que o coeficiente de $\left(y^{\prime \prime}\right)^{n-j_{*}}$ não se anula em $x^{\prime \prime}=0$, donde concluimos que $P^{\prime \prime}\left(0, y^{\prime \prime}\right)$ não pode ter uma raíz de multiplicidade $n$. Vamos escrever

$$
P^{\prime \prime}\left(0, y^{\prime \prime}\right)=f_{1}\left(y^{\prime \prime}\right) f_{2}\left(y^{\prime \prime}\right) g\left(y^{\prime \prime}\right)
$$

onde $f_{1}$ e $f_{2}$ são polinômio de graus menores que $n$ que não têm raízes em comum e $g$ é um polinômio sem raízes reais. Vamos assumir que $f_{1}, f_{2}$ e $g$ são mônicos.

Se $f_{1}$ e $f_{2}$ são constantes, então $P^{\prime \prime}\left(0, y^{\prime \prime}\right)$ tem somente raízes complexas. Como as raízes de um polinômio dependem continuamente de seus coeficientes, segue que $P^{\prime \prime}\left(x^{\prime \prime}, y^{\prime \prime}\right)$ tem somente raízes complexas, se $x^{\prime \prime}$ for suficientemente pequeno. Então

$$
\begin{aligned}
P\left(\left(x^{\prime \prime}\right)^{k}, y\right) & =\left(x^{\prime \prime}\right)^{n l} P^{\prime \prime}\left(x^{\prime \prime}, y^{\prime \prime}\right) \\
& =\left(x^{\prime \prime}\right)^{n l} P^{\prime \prime}\left(x^{\prime \prime}, \frac{y+\frac{1}{n} B_{1}\left(\left(x^{\prime \prime}\right)^{k}\right)}{\left(x^{\prime \prime}\right)^{l}}\right),
\end{aligned}
$$


onde o polinômio $P^{\prime \prime}$ (com relação a $y$ ) só tem raízes complexas para $x^{\prime \prime}$ pequeno, e a decomposição vale nesse caso.

Vamos assumir agora que os graus de $f_{1}, f_{2}$ e $g$ são menores que $n$. Pelo teorema anterior aplicado inicialmente à $f_{1} f_{2}, g$ e depois à $f_{1}, f_{2}$, existem polinômios em $y^{\prime \prime}, F_{1}, F_{2}$ e $G$ cujos coeficientes são funções analíticas de $x^{\prime \prime}$ tais que, para $x^{\prime \prime}$ pequeno,

$$
P^{\prime \prime}\left(x^{\prime \prime}, y^{\prime \prime}\right)=F_{1}\left(x^{\prime \prime}, y^{\prime \prime}\right) F_{2}\left(x^{\prime \prime}, y^{\prime \prime}\right) G\left(x^{\prime \prime}, y^{\prime \prime}\right),
$$

onde

$$
f_{1}\left(y^{\prime \prime}\right)=F_{1}\left(0, y^{\prime \prime}\right), f_{2}\left(y^{\prime \prime}\right)=F_{2}\left(0, y^{\prime \prime}\right), g\left(y^{\prime \prime}\right)=G\left(0, y^{\prime \prime}\right) .
$$

Novamente pelo fato de que as raízes de um polinômio dependem continuamente de seus coeficientes, como todas as raízes de $g\left(y^{\prime \prime}\right)$ são complexas, $G\left(x^{\prime \prime}, y^{\prime \prime}\right)$ também não tem raízes reais para $x^{\prime \prime}$ pequeno. Como os graus de $F_{1}$ e de $F_{2}$ são menores do que $n$, pela hipótese de indução existem $k_{1}, k_{2}, m_{1}, m_{2} \in \mathbb{N}$ tais que, em uma vizinhança de 0 ,

$$
\begin{gathered}
F_{1}\left(z^{k_{1}}, y^{\prime \prime}\right)=\left(y^{\prime \prime}-R_{1,1}(z)\right)\left(y^{\prime \prime}-R_{1,2}(z)\right) \ldots\left(y^{\prime \prime}-R_{1, m_{1}}(z)\right) G_{1}\left(z, y^{\prime \prime}\right), \\
F_{2}\left(w^{k_{2}}, y^{\prime \prime}\right)=\left(y^{\prime \prime}-R_{2,1}(w)\right)\left(y^{\prime \prime}-R_{2,2}(w)\right) \ldots\left(y^{\prime \prime}-R_{2, m_{2}}(w)\right) G_{2}\left(w, y^{\prime \prime}\right) .
\end{gathered}
$$

Sejam $p=k \cdot \operatorname{mmc}\left\{k_{1}, k_{2}\right\}$ e $a, b$ tais que $M=\operatorname{mmc}\left\{k_{1}, k_{2}\right\}=a . k_{1}=b . k_{2}$. Se $z=x^{a}, w=x^{b}$ e $x^{\prime \prime}=x^{M}$, então $\left(x^{\prime \prime}\right)^{n l}=x^{M n l} \mathrm{e}$

$$
P\left(x^{p}, y\right)=x^{M l n} F_{1}\left(x^{a k_{1}}, y^{\prime \prime}\right) \cdot F_{2}\left(x^{b k_{2}}, y^{\prime \prime}\right) G\left(x^{M}, y^{\prime \prime}\right) .
$$

Suponhamos que o grau de $G$ é $m$ com relação à $y^{\prime \prime}$. Como

$$
G\left(x^{M}, y^{\prime \prime}\right)=G\left(x^{M}, \frac{y+\frac{1}{n} B_{1}\left(x^{p}\right)}{x^{M l}}\right),
$$

então $x^{l \cdot m \cdot M} G\left(x^{M}, y^{\prime \prime}\right)$ é um polinômio em $y$ cujos coeficientes são funções analíticas de $x$ que não tem zeros se $x$ for pequeno. Como

$$
\begin{aligned}
& F_{1}\left(x^{M}, y^{\prime \prime}\right)=\left(y^{\prime \prime}-R_{1,1}\left(x^{a}\right)\right)\left(y^{\prime \prime}-R_{1,2}\left(x^{a}\right)\right) \ldots\left(y^{\prime \prime}-R_{1, m_{1}}\left(x^{a}\right)\right) G_{1}\left(x^{a}, y^{\prime \prime}\right) \\
& =\frac{1}{x^{M l . m_{1}}}\left(y-\left(x^{M l} R_{1,1}\left(x^{a}\right)-\frac{1}{n} B_{1}\left(x^{p}\right)\right)\right) \ldots G_{1}\left(x^{a}, \frac{y-\frac{1}{n} B_{1}\left(x^{p}\right)}{x^{M l}}\right),
\end{aligned}
$$

se o grau de $F_{1}$ é $s$, então $x^{\text {M.l.s }} F_{1}\left(x^{M}, y^{\prime \prime}\right)$ pode ser escrito como

$$
\left(y-S_{1}(x)\right) \ldots\left(y-S_{s_{1}}(x)\right) G_{1}^{\prime}(x, y),
$$

onde as funções $S_{i}$ são analíticas em $x$ e $G_{1}^{\prime}(x, y)$ é um polinômio em $y$ cujos coeficientes são funções analíticas de $x$ e para $x$ pequeno $G_{1}^{\prime}(x, y)$ não tem raízes reais.

Podemos escrever ainda $R_{i}^{\prime}(x)=R_{i}\left(x^{\frac{1}{p}}\right), G^{\prime}(x, y)=G\left(x^{\frac{1}{p}}, y\right)$,

$$
P(x, y)=\left(y-R_{1}^{\prime}(x)\right) \ldots\left(y-R_{m}^{\prime}(x)\right) G^{\prime}(x, y),
$$


onde para cada $i \leq m$, existe $n_{i} \in \mathbb{N}$ tal que

$$
R_{i}^{\prime}(x)=\sum_{j=n_{i}}^{\infty} c_{j}\left(x^{\frac{1}{p}}\right)^{j} .
$$

Em particular, se $R_{i}^{\prime} \neq 0$, podemos escrever que

$$
R_{i}^{\prime}(x)=c_{n_{i}} x^{\frac{n_{i}}{p}}(1+\mathrm{O}(1)), x \rightarrow 0 .
$$

Teorema 4.0.9 (Teorema de Puiseux) Seja $f$ uma função contínua, definida para $\xi \in \mathbb{R}$ positivo pequeno, tal que $y=f(\xi)$ satisfaz uma equação polinomial da forma

$$
A_{0}(\xi) y^{n}+A_{1}(\xi) y^{n-1}+\ldots+A_{n-1}(\xi) y+A_{n}(\xi)=0
$$

onde os coeficientes $A_{0}(\xi), \ldots, A_{n}(\xi)$ são funções analíticas. Então existem $p, q \in \mathbb{N}$ tais que a restrição de $f$ a algum intervalo do tipo $(0, \varepsilon)$ pode ser escrita como

$$
\xi^{q} f(\xi)=g\left(\xi^{\frac{1}{p}}\right)
$$

onde g é uma função analítica em um intervalo pequeno em torno da origem.

Demonstração: Vamos escrever que $A_{0}(\xi)=\xi^{q} B_{0}(\xi)$, onde $q \in \mathbb{N}$ e $B_{0}(\xi) \neq 0, \forall \xi \in \mathbb{R}$. Definindo $B_{j}(\xi)=\frac{A_{j}(\xi)}{B_{0}(\xi)}$, temos que

$$
\begin{aligned}
0 & =\xi^{q} y^{n}+B_{1}(\xi) y^{n-1}+\ldots+B_{n-1}(\xi) y+B_{n}(\xi) \\
& =\xi^{q-N n}\left(\xi^{N} y\right)^{n}+B_{1}(\xi) \xi^{-N(n-1)}\left(\xi^{N} y\right)^{n-1}+\ldots+B_{n-1}(\xi) \xi^{-N}\left(\xi^{N} y\right)+B_{n}(\xi) .
\end{aligned}
$$

Dividindo por $\xi^{q-N n}$ obtemos que

$$
0=\left(\xi^{N} y\right)^{n}+B_{1}(\xi) \xi^{N-q}\left(\xi^{N} y\right)^{n-1}+\ldots+B_{n-1}(\xi) \xi^{N(n-1)-q}\left(\xi^{N} y\right)+B_{n}(\xi) \xi^{N n-q}
$$

Logo, se $N=q$ a equação acima é igual a

$$
0=\left(\xi^{q} y\right)^{n}+B_{1}(\xi)\left(\xi^{q} y\right)^{n-1}+B_{2}(\xi) \xi^{q}\left(\xi^{q} y\right)^{n-2}+\ldots+B_{n-1}(\xi) \xi^{q(n-2)}\left(\xi^{q} y\right)+B_{n}(\xi) \xi^{q(n-1)}
$$

Pelo Teorema da Decomposição existem uma função $g$ analítica em um intervalo em torno do 0 e um natural $p$ tal que

$$
\xi^{q} f(\xi)=g\left(\xi^{\frac{1}{p}}\right)
$$

Nas condições do teorema anterior, se

$$
g(\xi)=\sum_{j=m}^{\infty} c_{j} \xi^{j},
$$

então

$$
f(\xi)=\sum_{j=m-p q}^{\infty} c_{j+p q}\left(\xi^{\frac{1}{p}}\right)^{j},
$$

e a menos que $f$ seja nula, podemos escrever que $f(\xi)=c_{m} \xi^{\frac{m-p q}{q}}(1+\mathrm{o}(1))$, se $\xi \rightarrow 0$. Podemos obter uma expressão semelhante para quando $\xi \rightarrow \infty$ fazendo

$$
f\left(\frac{1}{\xi}\right)=\sum_{j=m-p q}^{\infty} c_{j+p q}\left(\xi^{\frac{1}{-p}}\right)^{j} .
$$


Definição 4.0.5 Uma função $f: \mathbb{R} \rightarrow \mathbb{R}$ é dita algébrica se $y=f(x)$ for solução de uma equação do tipo

$$
P_{n}(x) y^{n}+\ldots+P_{1}(x) y+P_{0}(x)=0,
$$

onde cada $P_{j}$ é um polinômio.

Lema 4.0.9 Seja $A \subseteq \mathbb{R}$ um conjunto semi-algébrico. Então $A$ pode ser escrito como uma reunião finita de intervalos (onde vamos considerar que um ponto é um intervalo).

Demonstração: Podemos supor que $A$ é dado por uma intersecção finita de conjuntos do tipo $\left\{x \in \mathbb{R}: P_{1}(x)>0\right\}, \ldots,\left\{x \in \mathbb{R}: P_{k}(x)=0\right\}$, onde $k \geq 1$ e cada $P_{i}$ é um polinômio. Como cada um desses conjuntos se escreve como uma reunião finita de intervalos, segue o lema.

Vamos tratar agora do discriminante de um polinômio.

Definição 4.0.6 Um polinômio $p \in \mathbb{Z}\left[x_{1}, \ldots, x_{n}\right]$ é dito simétrico se

$$
p\left(x_{1}, \ldots, x_{i}, \ldots, x_{j}, \ldots, x_{n}\right)=p\left(x_{1}, \ldots, x_{j}, \ldots, x_{i}, \ldots, x_{n}\right), \forall 1 \leq i, j \leq n .
$$

Os exemplos mais importantes de polinômios simétricos são

$$
\begin{aligned}
& \sigma_{1}\left(x_{1}, \ldots, x_{n}\right)=x_{1}+\ldots+x_{n} \\
& \sigma_{2}\left(x_{1}, \ldots, x_{n}\right)=\left(x_{1} x_{2}+\ldots+x_{1} x_{n}\right)+\left(x_{2} x_{3}+\ldots+x_{2} x_{n}\right)+\ldots+\left(x_{n-1} x_{n}\right) \\
& \ldots \\
& \sigma_{n}\left(x_{1}, \ldots, x_{n}\right)=x_{1} \ldots x_{n} .
\end{aligned}
$$

Pode-se provar o seguinte resultado bastante importante:

Teorema 4.0.10 Seja $p \in \mathbb{Z}\left[x_{1}, \ldots, x_{n}\right]$ um polinômio simétrico. Então existe exatamente um polinômio $Q$ de $n$ váriáveis com coeficientes inteiros tal que

$$
p\left(x_{1}, \ldots, x_{n}\right)=Q\left(\sigma_{1}\left(x_{1}, \ldots, x_{n}\right), \ldots, \sigma_{n}\left(x_{1}, \ldots, x_{n}\right)\right) .
$$

Outro polinômio simétrico muito importante é o dado pelo determinante de Vandermonde:

$$
D\left(x_{1}, \ldots, x_{n}\right)=\prod_{i<j}\left(x_{i}-x_{j}\right)^{2} .
$$

Vale notar que $D\left(x_{1}, \ldots, x_{n}\right)=0$ se, e somente se, existem $i$ e $j$ distintos com $x_{i}=x_{j}$.

Definição 4.0.7 Seja $p(x)=(-1)^{n} a_{n}+\ldots+a_{2} x^{n-2}-a_{1} x^{n-1}+x^{n}$ um polinômio com coeficientes complexos.

Sabemos que existe exatamente um polinômio $Q \in \mathbb{Z}\left[x_{1}, \ldots, x_{n}\right]$ tal que

$$
D\left(x_{1}, \ldots, x_{n}\right)=Q\left(\sigma_{1}\left(x_{1}, \ldots, x_{n}\right), \ldots, \sigma_{n}\left(x_{1}, \ldots, x_{n}\right)\right) .
$$

Então $\triangle(p)=Q\left(a_{1}, \ldots, a_{n}\right)$ é chamado o discriminante de $p$.

Outro resultado bastante útil é o seguinte: 
Teorema 4.0.11 Seja $p(x)=\prod_{j=1}^{n}\left(x-x_{j}\right) \in \mathbb{C}[x]$. Então $p$ tem uma raíz múltipla se, e somente se, $\triangle(p)=0$. Além disso,

$$
\triangle(p)=\prod_{i<j}\left(x_{i}-x_{j}\right)^{2},
$$

e $p(x)=(-1)^{n} a_{n}+\ldots+a_{2} x^{n-2}-a_{1} x^{n-1}+x^{n}$, onde

$$
a_{k}=\sigma_{k}\left(x_{1}, \ldots, x_{n}\right), \forall k \text {. }
$$

Teorema 4.0.12 Seja $f$ uma função semi-algébrica definida em $\mathbb{R}$. Então $\mathbb{R}$ pode ser decomposto em um número finito de intervalos (onde consideramos que um intervalo pode ser só um ponto) nos quais $f=\infty, f=-\infty$ ou $f$ é igual a uma função algébrica e contínua. Além disso, se $f$ for finita para $x$ positivo suficientemente grande, e não identicamente nula, então existem constantes $C \neq 0, q \in \mathbb{Q}$ tais que

$$
f(x)=C x^{q}(1+\mathrm{O}(1)), x \rightarrow \infty .
$$

Demonstração: Vimos que $f$ é semi-algébrica se, e somente se, seu sub-gráfico for semi-algébrico. Como seu sub-gráfico, isto é, o conjunto $F=\left\{(x, y) \in \mathbb{R}^{2}: y \leq f(x)\right\}$ é semi-algébrico, então sua projeção em $\mathbb{R}$ é semi-algébrica, isto é, o conjunto

$$
A=\{x \in \mathbb{R}: \exists y \in \mathbb{R}: y \leq f(x)\}
$$

é semi-algébrico. Pelo lema 4.0.9 ele pode ser escrito como uma reunião finita de intervalos. Como o complementar da projeção é semi-algébrico, ele também é uma reunião finita de intervalos. Sabemos ainda que

$$
A^{c}=\{x \in \mathbb{R}: \forall y \in \mathbb{R}, f(x)<y\}=\{x \in \mathbb{R}: f(x)=-\infty\} .
$$

Como $F^{c}$ é semi-algébrico, sua projeção em $\mathbb{R}$ é semi-algébrica, donde o complementar dessa projeção também o é, e portanto se escreve como uma reunião finita de intervalos. Notemos que a projeção em $\mathbb{R}$ de $F^{c}$ é o conjunto

$$
B=\{x \in \mathbb{R}: \exists y \in \mathbb{R}: f(x)<y\}
$$

Assim,

$$
B^{c}=\{x \in \mathbb{R}: f(x)=\infty\} .
$$

Temos que $A=\{x \in \mathbb{R}: f(x) \in \mathbb{R}\} \cup\{x \in \mathbb{R}: f(x)=\infty\}$ pode ser escrito como uma reunião finita de intervalos e $B=\{x \in \mathbb{R}: \exists y \in \mathbb{R}: f(x)<y\}$ também. Digamos que $A=\cup_{i=1}^{m} L_{i}$, $B=\cup_{j=1}^{s} I_{j}$. Então

$$
\{x \in \mathbb{R}: f(x) \in \mathbb{R}\}=\left(\cup_{j=1}^{s} I_{j}\right) \cap\left(\cup_{i=1}^{m} L_{i}\right)=\cup_{i, j}\left(I_{j} \cap L_{i}\right),
$$

portanto $\{x \in \mathbb{R}: f(x) \in \mathbb{R}\}$ se escreve como uma reunião finita de intervalos. Digamos que $F$ seja definido por

$$
P_{1}(x, y)>0, \ldots, P_{r}(x, y)=0 .
$$

Seja $I$ um intervalo aberto no qual $f$ é finita. Considere $g(x, y)=P_{1}(x, y) \ldots P_{r}(x, y)$. O discriminante de $g(x,$.$) é um polinômio em x$; tirando os seus zeros que estão em $I$ obteremos novos intervalos nos quais $\triangle(g) \neq 0$, para todo $x$ nesses intervalos. Seja $I^{\prime}$ um dos novos (finitos) 
intervalos contidos em $I$ obtidos dessa forma. Segue do teorema anterior que em $I^{\prime}$ as equações $P_{j}(x, y)=0$ definem um número finito de funções $y=f_{j}(x)$ tais que $f_{1}(x)<f_{2}(x)<\ldots<f_{l}(x)$. Sejam

$$
C_{i}=\left\{(x, y): x \in I^{\prime}, y \in \mathbb{R}: f_{i}(x)<y<f_{i+1}(x)\right\} .
$$

Dessa forma podemos afirmar que ou todo $(x, y) \in C_{i}$ satisfaz (4.0.3), ou nenhum $(x, y) \in C_{i}$ satisfaz (4.0.3), pois $C_{i}$ é um conjunto conexo no qual nenhum $P_{j}$ tem zero, e no qual os $P_{j}$ não mudam de sinal (se em (4.0.3) aparecer um polinômio $P_{j}$ como $P_{j}(x, y)=0$, então nenhum ponto de $C_{i}$ satisfaz (4.0.3)). De modo similar, os seguintes conjuntos ou estão contidos em $F$, ou são disjuntos de $F$ :

$$
\left\{\left(x, f_{j}(x)\right): x \in I^{\prime}\right\},\left\{(x, y): x \in I^{\prime}, y<f_{1}(x)\right\}
$$

A seguir veremos que podemos concluir que existe algum $i$ tal que $f=f_{i}$ em $I^{\prime}$.

Vejamos inicialmente porque dado $x_{0} \in I^{\prime}$, existe $i$ (que a princípio poderia depender de $x_{0}$ ) tal que $f\left(x_{0}\right)=f_{i}\left(x_{0}\right)$. Se $f\left(x_{0}\right)<f_{1}\left(x_{0}\right)$, como $\left(x_{0}, f\left(x_{0}\right)\right) \in F$ teríamos que todo $y$ tal que $y<f_{1}\left(x_{0}\right)$ é tal que $\left(x_{0}, y\right) \in F$, um absurdo, pois existe $y_{0}$ tal que $f\left(x_{0}\right)<y_{0}<f_{1}\left(x_{0}\right)$, e portanto $\left(x_{0}, y_{0}\right) \notin F$. Analogamente, não pode existir $i$ tal que $f_{i}\left(x_{0}\right)<f\left(x_{0}\right)<f_{i+1}\left(x_{0}\right)$ pois teríamos por um lado que $C_{i} \subseteq F$, mas por outro existiria $y: f_{i}\left(x_{0}\right)<f\left(x_{0}\right)<y<f_{i+1}\left(x_{0}\right)$, isto é, $\left(x_{0}, y\right) \notin F$. Finalmente, se $f_{k}\left(x_{0}\right)<f\left(x_{0}\right)$, por um lado teríamos que $\left\{(x, y): f_{k}(x)<y\right\} \subseteq F$, mas por outro lado existe $y$ tal que $f_{k}\left(x_{0}\right)<f\left(x_{0}\right)<y$ e portanto $\left(x_{0}, y\right) \notin F$.

Vejamos agora porque o $i$ não depende de $x$. Suponhamos por absurdo que existam $x, z \in I^{\prime}$ tais que $f(x)=f_{i}(x), f(z)=f_{j}(z)$, com $i<j$. Seja $y$ tal que $f_{j-1}(x)<y<f_{j}(x)$. Como $f(z)=f_{j}(z)$, segue que $\left\{(w, v): w \in I^{\prime}, v \in \mathbb{R}, f_{j-1}(w)<v<f_{j}(w)\right\} \subseteq F$, e portanto $(x, y) \in F$, donde $f_{j-1}(x)<f(x)$, um absurdo, pois $f_{i}(x) \leq f_{j-1}(x)$.

Em particular, se $f$ for finita não nula para $x$ suficientemente grande, então existe $i$ tal que $f=f_{i}$ em um intervalo ilimitado à direita. Pelo Teorema de Puiseux existem constantes $C \neq 0, q \in \mathbb{Q}$ tais que

$$
f(x)=f_{i}(x)=C x^{q}(1+\mathrm{o}(1)), x \rightarrow \infty .
$$

Corolário 4.0.4 Seja $E \subseteq \mathbb{R}^{2+n}$ um conjunto semi-algébrico tal que

$$
f(x)=\inf \left\{y \in \mathbb{R}: \exists z \in \mathbb{R}^{n}:(x, y, z) \in E\right\}
$$

está definida e é finita para $x$ suficientemente grande. Então ou $f(x)=0$ para todo $x$ grande, ou existem $C \neq 0, q \in \mathbb{Q}$ tais que

$$
f(x)=C x^{q}(1+\mathrm{o}(1)), x \rightarrow \infty .
$$

Demonstração: Vimos que como $E$ é semi-algébrico, $f$ é semi-algébrica. O resultado segue então imediatamente do teorema anterior.

Vale notar que (4.0.4) também vale se $x \rightarrow 0$, e também se definirmos $f$ como

$$
f(x)=\sup \left\{y \in \mathbb{R}: \exists z \in \mathbb{R}^{n}:(x, y, z) \in E\right\} .
$$




\section{Capítulo 5}

\section{Lema algébrico}

Antes de provarmos o lema (1.0.1), vejamos como é a prova desse resultado no caso unidimensional.

Lema 5.0.10 Sejam $Q$ um polinômio em $\mathbb{R}$ e $\mathcal{N}$ o conjunto de seus zeros. Se $\exists x \in \mathcal{N}$ tal que $|x| \leq 1$, então existem constantes positivas $C, l$ tais que

$$
C \operatorname{dist}(x, \mathcal{N})^{l} \leq|Q(x)|, \forall x \in[-1,1] .
$$

Demonstração: Escreva

$$
Q(x)=\left(x-x_{1}\right)^{l_{1}} \ldots .\left(x-x_{p}\right)^{l_{p}} q(x),
$$

onde $\left\{x_{1}, \ldots, x_{p}\right\}=\{x \in[-1,1]: Q(x)=0\}, l_{i} \geq 1, p \geq 1$ e $q(x) \neq 0, \forall x \in[-1,1]$. Seja $r>0$ tal que $B_{r}\left[x_{i}\right] \cap B_{r}\left[x_{j}\right]=\emptyset$ se $i \neq j$. Seja $M>0$ tal que $M \leq|q(x)|, \forall x \in[-1,1]$. Sejam ainda $R=\min \left\{r, \frac{1}{2}\right\}, l=\max \left\{l_{1}, \ldots, l_{p}\right\}$,

$$
N=\min \left\{|Q(x)|: x \in[-1,1] \cap\left(\cup_{i=1}^{p} B_{R}\left(x_{i}\right)\right)^{c}\right\} .
$$

Se $x \in B_{R}\left[x_{j}\right]$ e $x \in[-1,1]$, então

$$
\begin{aligned}
|Q(x)| & =\left|x-x_{1}\right|^{l_{1}} \ldots\left|x-x_{p}\right|^{l_{p}}|q(x)| \\
& \geq R^{l_{1}} \ldots R^{l_{j-1}} R^{l_{j+1}} \ldots R^{l_{p}}\left|x-x_{j}\right|^{l_{j}} \cdot M \\
& =M \cdot R^{l_{1}+\ldots+l_{j-1}+l_{j+1}+\ldots+l_{p}} \operatorname{dist}(x, \mathcal{N})^{l_{j}} \\
& \geq M \cdot R^{l . p} \operatorname{dist}(x, \mathcal{N})^{l} \\
& =C_{1} \operatorname{dist}(x, \mathcal{N})^{l} .
\end{aligned}
$$

Se $x \in[-1,1]$ e $x \notin \cup_{i=1}^{p} B_{R}\left(x_{i}\right)$, então

$$
\frac{N}{2^{l}} \operatorname{dist}(x, \mathcal{N})^{l} \leq \frac{N}{2^{l}} \cdot 2^{l}=N \leq|Q(x)| .
$$

Basta definir $C=\min \left\{C_{1}, \frac{N}{2^{l}}\right\}$.

Vejamos agora a versão do resultado anterior para um polinômio em $m$ variáveis. 
Lema 5.0.11 Sejam $Q$ um polinômio em $\mathbb{R}^{m}$ e $\mathcal{N}$ o conjunto de seus zeros. Se existir $\xi \in \mathcal{N}:$ $|\xi| \leq 1$, então existem constantes positivas $C, \mu$ tais que

$$
C \operatorname{dist}(\xi, \mathcal{N})^{\mu} \leq|Q(\xi)|, \forall|\xi| \leq 1 .
$$

Vale observar que se $\mathcal{N} \neq \emptyset$ e $\mathcal{N} \cap B_{1}[0]=\emptyset$, então esse resultado é imediato. De fato, se $M>0$ é tal que $M \leq|Q(\xi)|, \forall \xi \in B_{1}[0]$, e se $K>0$ for tal que $\operatorname{dist}(\xi, \mathcal{N}) \leq K, \forall \xi \in B_{1}[0]$, então

$$
\frac{M}{K} \operatorname{dist}(\xi, \mathcal{N}) \leq M \leq|Q(\xi)|, \forall \xi \in B_{1}[0] .
$$

Demonstração: Vamos estudar inicialmente as condições sobre $\xi$ de modo que, se $\delta>0$,

$$
\operatorname{dist}(\xi, \mathcal{N})<\delta
$$

Temos que $\xi$ satisfaz (5.0.1) se, e somente se,

$$
\exists \eta \in \mathbb{R}^{m}: Q(\eta)=0 \text { e }|\eta-\xi|^{2}<\delta^{2} .
$$

Seja então

$$
A=\left\{(\delta, \xi, \eta) \in \mathbb{R} \times \mathbb{R}^{2 m}: \delta>0, Q(\eta)=0,|\eta-\xi|^{2}<\delta^{2}\right\} .
$$

Como $A$ é um conjunto semi-algébrico, decorre do Teorema de Seidenberg-Tarski que o seguinte conjunto também é semi-algébrico:

$$
\left\{(\delta, \xi) \in \mathbb{R} \times \mathbb{R}^{m}: \exists \eta \in \mathbb{R}^{m}:(\delta, \xi, \eta) \in A\right\} .
$$

Assim existe um número finito de conjuntos $G_{1}(\delta, \xi), \ldots, G_{r}(\delta, \xi)$, cada um deles escrito por igualdades ou desigualdades polinomiais, tais que (5.0.2) vale se, e somente se, $(\delta, \xi)$ satisfizer as equações que definem $G_{i}(\delta, \xi)$, para algum $i \leq r$.

Sejam $H_{1}(\delta, \xi), \ldots H_{s}(\delta, \xi)$ os conjuntos obtidos da seguinte forma: primeiramente negamos uma condição em cada $G_{i}(\delta, \xi)$ e depois interceptamos esses novos conjuntos. Dessa forma tere$\operatorname{mos}$ que $\operatorname{dist}(\xi, \mathcal{N}) \geq \delta$ se, e somente se, $(\delta, \xi)$ for elemento de ao menos um $H_{j}(\delta, \xi)$.

Consideramos agora os valores de $|Q(\xi)|$ quando $\operatorname{dist}(\xi, \mathcal{N}) \geq \delta$ e $|\xi| \leq 1$. Temos que $\tau=$ $|Q(\xi)|$, para algum $\xi$ tal que $\operatorname{dist}(\xi, \mathcal{N}) \geq \delta$ e $|\xi| \leq 1$ se, e somente se,

$$
\exists \xi \in \mathbb{R}^{m}: \tau^{2}=|Q(\xi)|^{2}, \tau>0,|\xi|^{2} \leq 1,(\delta, \xi) \in H_{j}(\delta, \xi) \text { para ao menos um } j \leq s .
$$

Seja

$$
T(\delta)=\inf \{|Q(\xi)|:|\xi| \leq 1, \operatorname{dist}(\xi, \mathcal{N}) \geq \delta\},
$$

isto é, $T(\delta)$ é o ínfimo de $|Q|$ no compacto $K=\{\xi:|\xi| \leq 1$, $\operatorname{dist}(\xi, \mathcal{N}) \geq \delta)\}$, que não é vazio se $\delta$ for pequeno e $Q$ não for nulo. Podemos ver que $T$ é uma função crescente.

Veremos que existe $\delta_{0}>0$ tal que $T$ algébrica em $] 0, \delta_{0}[$. Seja

$$
B=\left\{(\delta, \tau, \xi) \in \mathbb{R}^{2+m}: \tau>0, \tau^{2}=|Q(\xi)|^{2},|\xi|^{2} \leq 1,(\delta, \xi) \in H_{j}(\delta, \xi) \text { para algum } j \leq s\right\} .
$$


Como $B$ é semi-algébrico e $T(\delta)=\inf \left\{\tau \in \mathbb{R}: \exists \xi \in \mathbb{R}^{m}:(\delta, \tau, \xi) \in B\right\}$, segue pelo Corolário 3.0.3 que $T$ é semi-algébrica. Prosseguindo como na prova do teorema 4.0.12 provamos que existe $\delta_{0}>0$ tal que, em $\left(0, \delta_{0}\right), T$ é igual a uma função algébrica e contínua.

Tudo foi feito para que nesse momento possamos utilizar o Teorema de Puiseux. Por esse teorema podemos escrever, para $\delta$ pequeno, que

$$
T(\delta)=d \delta^{\mu}(1+\mathrm{O}(1))
$$

onde $d$ e $\mu \in \mathbb{Q}$ são positivos pela definição de $T$. Dessa forma existe $\delta^{\prime}>0$ tal que $0<\delta \leq \delta^{\prime}$ implica

$$
\frac{d}{2} \delta^{\mu} \leq T(\delta)
$$

Assim o lema vale com $C=\frac{d}{2}$ se $|\xi| \leq 1$ e $0<\operatorname{dist}(\xi, \mathcal{N})<\delta^{\prime}$ pois fazendo $\delta=\operatorname{dist}(\xi, \mathcal{N})$ em (5.0.4) vem:

$$
\begin{aligned}
C \operatorname{dist}(\xi, \mathcal{N})^{\mu} & \leq T(\operatorname{dist}(\xi, \mathcal{N})) \\
& =\inf \{|Q(z)|:|z| \leq 1, \operatorname{dist}(z, \mathcal{N}) \geq \operatorname{dist}(\xi, \mathcal{N})\} \\
& \leq|Q(\xi)|
\end{aligned}
$$

Obviamente o lema vale se $|\xi| \leq 1$ e $\operatorname{dist}(\xi, \mathcal{N})=0$. Resta ver se $|\xi| \leq 1$ e $\operatorname{dist}(\xi, \mathcal{N}) \geq \delta^{\prime}$. Seja $M>0$ tal que $M \leq|Q(\xi)|, \forall \xi \in B_{1}[0]$ tais que $\delta^{\prime} \leq \operatorname{dist}(\xi, \mathcal{N})$. Como $\operatorname{dist}(\xi, \mathcal{N})^{\mu} \leq 2^{\mu}, \forall \xi \in$ $B_{1}[0]$, então

$$
\frac{M}{2^{\mu}} \operatorname{dist}(\xi, \mathcal{N})^{\mu} \leq M \leq|Q(\xi)|, \forall \xi \in B_{1}[0]: \delta^{\prime} \leq \operatorname{dist}(\xi, \mathcal{N}) .
$$

Definimos então $C=\min \left\{\frac{d}{2}, \frac{M}{2^{\mu}}\right\}$.

Convém agora excluirmos a hipótese de que $|\xi| \leq 1$.

Teorema 5.0.13 Sejam $Q$ um polinômio em $\mathbb{R}^{n}$ e $\mathcal{N}$ o conjunto dos seus zeros. Então ou $\mathcal{N}=\emptyset$ e existem constantes $C, a>0$ tais que

$$
C\left(1+|\xi|^{2}\right)^{-a} \leq|Q(\xi)|, \forall \xi \in \mathbb{R}^{n},
$$

ou $\mathcal{N} \neq \emptyset$ e existem constantes $C, a, b>0$ tais que

$$
C\left(1+|\xi|^{2}\right)^{-a} \operatorname{dist}(\xi, \mathcal{N})^{b} \leq|Q(\xi)|, \forall \xi \in \mathbb{R}^{n} .
$$

Demonstração: Notemos que se $Q$ for constante o resultado é imediato. Vamos analisar inicialmente o caso em que $|\xi|>1$. Seja $\xi^{*}=\frac{\xi}{|\xi|^{2}}$. Assim sendo, $\left|\xi^{*}\right|^{2}=\frac{1}{|\xi|^{2}}$ e $\xi=\frac{\xi^{*}}{\left|\xi^{*}\right|^{2}}$.

Se o grau de $Q$ é $m \geq 1$, seja

$$
Q^{*}\left(\xi^{*}\right)=\left|\xi^{*}\right|^{2 m} Q(\xi)=\left|\xi^{*}\right|^{2 m} Q\left(\frac{\xi^{*}}{\left|\xi^{*}\right|^{2}}\right) .
$$

Seja $\mathcal{N}^{*}$ o conjunto dos zeros de $Q^{*}$. Temos que $0 \neq \xi^{*} \in \mathcal{N}^{*}$ se, e somente se, $\xi \in \mathcal{N}$. Além disso $0 \in \mathcal{N}^{*}$ e portanto podemos aplicar o lema anterior. Conseqüentemente, existem constantes $C^{*}, \mu^{*}>0$ tais que

$$
\left|Q^{*}\left(\xi^{*}\right)\right| \geq C^{*} \operatorname{dist}\left(\xi^{*}, \mathcal{N}^{*}\right)^{\mu^{*}}, \forall \xi^{*}:\left|\xi^{*}\right| \leq 1 .
$$


Dessa forma,

$$
\left|\xi^{*}\right|^{2 m}|Q(\xi)|=|\xi|^{-2 m}|Q(\xi)| \geq C^{*} \operatorname{dist}\left(\xi^{*}, \mathcal{N}^{*}\right)^{\mu^{*}}, \forall \xi:|\xi| \geq 1 .
$$

Notemos que $|\theta-\rho|^{2}=|\theta|^{2}+|\rho|^{2}-2<\theta, \rho>$. Assim,

$$
\left.|\eta| \xi\right|^{2}-\left.\xi|\eta|^{2}\right|^{2}=|\xi|^{4}|\eta|^{2}+|\xi|^{2}|\eta|^{4}-2|\xi|^{2}|\eta|^{2}<\eta, \xi>
$$

Por sua vez, $|\xi-\eta|^{2}=|\xi|^{2}+|\eta|^{2}-2<\xi, \eta>$, e assim

$$
|\xi|^{2}|\eta|^{2}|\xi-\eta|^{2}=|\xi|^{4}|\eta|^{2}+|\xi|^{2}|\eta|^{4}-2|\xi|^{2}|\eta|^{2}<\xi, \eta>.
$$

Disso segue que $\left.|\eta| \xi\right|^{2}-\left.\xi|\eta|^{2}\right|^{2}=|\xi|^{2}|\eta|^{2}|\xi-\eta|^{2}$.

$$
\begin{aligned}
\frac{|\eta-\xi|}{\left|\eta^{*}-\xi^{*}\right|} & =\frac{|\eta-\xi|}{\left|\frac{\eta}{|\eta|^{2}}-\frac{\xi}{|\xi|^{2}}\right|} \\
& =\frac{|\eta-\xi|}{\left.|\eta| \xi\right|^{2}-\xi|\eta|^{2} \mid}|\eta|^{2}|\xi|^{2} \\
& =\frac{|\eta-\xi|}{|\xi \eta||\xi-\eta|^{2}}|\eta|^{2}|\xi|^{2} \\
& =|\eta||\xi| .
\end{aligned}
$$

Assim

$$
|\xi|^{2}\left|\eta^{*}-\xi^{*}\right|=|\xi| \frac{|\xi-\eta|}{|\eta|}, \forall \xi, \forall \eta \neq 0
$$

Pelo visto acima teremos que para todo $\xi \neq 0$,

$$
|\xi|^{2} \operatorname{dist}\left(\xi^{*}, \mathcal{N}^{*}\right)=\inf _{\eta^{*} \in \mathcal{N}^{*}}|\xi|^{2}\left|\eta^{*}-\xi^{*}\right|=\inf \left\{|\xi|,|\xi| \frac{|\xi-\eta|}{|\eta|}: \eta \in \mathcal{N} \backslash\{0\}\right\} .
$$

Suponhamos que $\mathcal{N} \neq \emptyset$. Sejam $\xi, \eta$ tais que $|\xi| \geq 1, \eta \in \mathcal{N},|\eta| \leq 2|\xi|$ e $\eta \neq 0$. Temos que $\operatorname{dist}(\xi, \mathcal{N}) \leq|\xi-\eta|$ e $\frac{1}{2|\xi|} \leq \frac{1}{|\eta|}$, donde $\frac{1}{2|\xi|^{2}} \leq \frac{1}{|\xi||\eta|}$ e portanto

$$
\frac{\operatorname{dist}(\xi, \mathcal{N})}{2|\xi|^{2}} \leq \frac{|\eta-\xi|}{|\eta||\xi|}, \forall \xi, \eta: \eta \in \mathcal{N},|\xi| \geq 1,|\eta| \leq 2|\xi|, \eta \neq 0
$$

Vejamos que

$$
\frac{1}{2|\xi|} \leq \frac{|\eta-\xi|}{|\eta||\xi|}, \forall \xi, \eta:|\xi| \geq 1,2|\xi| \leq|\eta|
$$

Seja $\zeta=\frac{\eta}{|\xi|}$. Então para provar (5.0.8) é suficiente mostrar que

$$
\frac{|\zeta|}{2} \leq|\zeta-w|, \forall \zeta, w:|\zeta| \geq 2,|w|=1
$$

Mas

$$
|\zeta-w|^{2}=|\zeta|^{2}-2(\zeta \cdot w)+1=\frac{|\zeta|^{2}}{4}+A
$$

onde

$$
A=\frac{3|\zeta|^{2}}{4}-2(\zeta \cdot w)+1 \geq \frac{3|\zeta|^{2}}{4}-2|\zeta|+1
$$


pois, como $|w|=1,(\zeta \cdot w) \leq|\zeta|$. A equação (5.0.9) é equivalente a $A \geq 0$, o que é verdade pois as raízes de $\frac{3 t^{2}}{4}-2 t+1$ são $\frac{2}{3}$ e 2 e portanto $A \geq 0$ se $|\zeta| \geq 2$.

Vejamos agora que existe uma constante $C_{1}>0$ tal que $\operatorname{dist}(\xi, \mathcal{N}) \leq C_{1}|\xi|, \forall|\xi| \geq 1$. De fato, tome $\xi_{0} \in \mathcal{N}$ e defina $C_{1}=1+\left|\xi_{0}\right|$. Se $|\xi| \geq 1$,

$$
\operatorname{dist}(\xi, \mathcal{N}) \leq\left|\xi-\xi_{0}\right| \leq|\xi|+\left|\xi_{0}\right| \leq C_{1}|\xi| .
$$

Afirmamos que existe $C>0$ tal que $\forall|\xi| \geq 1$,

$$
\operatorname{dist}(\xi, \mathcal{N}) \leq C|\xi|^{2} \operatorname{dist}\left(\xi^{*}, \mathcal{N}^{*}\right)
$$

De fato, se $|\xi| \geq 1,0 \neq \eta \in \mathcal{N}$ e $|\eta| \leq 2|\xi|$, então por (5.0.7),

$$
\operatorname{dist}(\xi, \mathcal{N}) \leq 2 \frac{|\xi|^{2}|\eta-\xi|}{|\eta||\xi|}
$$

Analogamente, pelas equações (5.0.8) e (5.0.10) concluimos que se $|\xi| \geq 1$ e $|\eta| \geq 2|\xi|$, então

$$
\frac{|\xi|^{2}|\eta-\xi|}{|\eta||\xi|} \geq \frac{|\xi|}{2} \geq \frac{\operatorname{dist}(\xi, \mathcal{N})}{2 C_{1}}
$$

Assim sendo, $\forall \xi$ tal que $|\xi| \geq 1, \forall \eta \in \mathcal{N}$ tal que $0 \neq \eta$,

$$
\operatorname{dist}(\xi, \mathcal{N}) \leq\left(2 C_{1}+2\right) \frac{|\xi|^{2}|\eta-\xi|}{|\eta||\xi|}
$$

Seja $C=\max \left\{2 C_{1}+2, C_{1}\right\}$, onde $\operatorname{dist}(\xi, \mathcal{N}) \leq C_{1}|\xi|, \forall|\xi| \geq 1$. Pela equação (5.0.6) concluimos que $\operatorname{dist}(\xi, \mathcal{N}) \leq C|\xi|^{2} \operatorname{dist}\left(\xi^{*}, \mathcal{N}^{*}\right), \forall \xi$ tal que $|\xi| \geq 1$.

Pela equação (5.0.5) e usando a última desigualdade concluimos que

$$
|Q(\xi)| \geq C^{*}|\xi|^{2 m} \operatorname{dist}\left(\xi^{*}, \mathcal{N}^{*}\right)^{\mu^{*}} \geq C^{*} C^{-\mu^{*}}|\xi|^{2 m}|\xi|^{-2 \mu^{*}} \operatorname{dist}(\xi, \mathcal{N})^{\mu^{*}}, \forall \xi:|\xi| \geq 1 .
$$

Afirmamos que

$$
\frac{1}{\left(1+|\xi|^{2}\right)^{\left|m-\mu^{*}\right|}} \leq|\xi|^{2 m-2 \mu^{*}}, \forall \xi:|\xi| \geq 1 .
$$

Se $\left|m-\mu^{*}\right|=m-\mu^{*}$, então

$$
\frac{1}{\left(1+|\xi|^{2}\right)^{m-\mu^{*}}} \leq 1 \leq|\xi|^{2 m-2 \mu^{*}}
$$

se, por outro lado, $\left|m-\mu^{*}\right|=\mu^{*}-m$, então

$$
|\xi|^{2 \mu^{*}-2 m}=|\xi|^{2\left(\mu^{*}-m\right)} \leq\left(1+|\xi|^{2}\right)^{\mu^{*}-m} .
$$

Assim,

$$
|Q(\xi)| \geq C^{*} C^{-\mu^{*}}\left(1+|\xi|^{2}\right)^{-\left|m-\mu^{*}\right|} \operatorname{dist}(\xi, \mathcal{N})^{\mu^{*}}, \forall \xi:|\xi| \geq 1 .
$$

Pelo lema anterior existem constantes $C_{2}, \mu^{\prime}>0$ tais que

$$
|Q(\xi)| \geq C_{2} \operatorname{dist}(\xi, \mathcal{N})^{\mu^{\prime}} \geq C_{2}\left(1+|\xi|^{2}\right)^{-\left|m-\mu^{*}\right|} \operatorname{dist}(\xi, \mathcal{N})^{\mu^{\prime}}, \forall \xi:|\xi| \leq 1 .
$$


Em resumo, existem constantes $K_{1}, K_{2}, a_{1}, a_{2}, b>0$ tais que

$$
\begin{aligned}
& |Q(\xi)| \geq K_{1} \operatorname{dist}(\xi, \mathcal{N})^{a_{1}}\left(1+|\xi|^{2}\right)^{-b}, \forall|\xi| \leq 1, \\
& |Q(\xi)| \geq K_{2} \operatorname{dist}(\xi, \mathcal{N})^{a_{2}}\left(1+|\xi|^{2}\right)^{-b}, \forall|\xi| \geq 1 .
\end{aligned}
$$

Nosso objetivo é encontrar constantes que sirvam para os dois casos. Sejam $y \in \mathcal{N}$ e $\lambda=2(1+|y|)$. Então se $a=\max \left\{a_{1}, a_{2}\right\}, K=\min \left\{K_{1} \lambda^{a_{1}-a}, K_{2} \lambda^{a_{2}-a}\right\}$,

$$
\begin{aligned}
K_{i} \operatorname{dist}(\xi, \mathcal{N})^{a_{i}}\left(\frac{1}{1+|\xi|^{2}}\right)^{b} & \geq K_{i} \operatorname{dist}(\xi, \mathcal{N})^{a_{i}}\left(\frac{1}{1+|\xi|^{2}}\right)^{b+a_{1}+a_{2}} \\
& =K_{i} \cdot \lambda^{a_{i}}\left(\frac{\operatorname{dist}(\xi, \mathcal{N})}{\lambda\left(1+|\xi|^{2}\right)}\right)^{a_{i}}\left(\frac{1}{1+|\xi|^{2}}\right)^{b+a_{1}+a_{2}-a_{i}} \\
& \geq K_{i} \cdot \lambda^{a_{i}}\left(\frac{\operatorname{dist}(\xi, \mathcal{N})}{\lambda\left(1+|\xi|^{2}\right)}\right)^{a}\left(\frac{1}{1+|\xi|^{2}}\right)^{b+a_{1}+a_{2}-a_{i}} \\
& =K_{i} \lambda^{a_{i}-a} \operatorname{dist}(\xi, \mathcal{N})^{a}\left(\frac{1}{1+|\xi|^{2}}\right)^{b+a_{1}+a_{2}-a_{i}+a} \\
& \geq K \operatorname{dist}(\xi, \mathcal{N})^{a}\left(\frac{1}{1+|\xi|^{2}}\right)^{b+a_{1}+a_{2}+a}
\end{aligned}
$$

Se $\mathcal{N}=\emptyset$, então $\mathcal{N}^{*}=\{0\}$ e portanto $\operatorname{dist}\left(\xi^{*}, \mathcal{N}^{*}\right)=\left|\xi^{*}\right|=\frac{1}{|\xi|}$. Pela equação (5.0.5) concluimos que

$$
|Q(\xi)| \geq C^{*}|\xi|^{2 m} \operatorname{dist}\left(\xi^{*}, \mathcal{N}^{*}\right)^{\mu^{*}}=C^{*}|\xi|^{2 m-2 \mu^{*}}, \forall \xi:|\xi| \geq 1
$$

Assim

$$
|Q(\xi)| \geq C^{*} \frac{1}{\left(1+|\xi|^{2}\right)^{\left|m-\mu^{*}\right|}}, \forall \xi:|\xi| \geq 1 .
$$

Como $\mathcal{N}=\emptyset$, existe uma constante $K>0$ tal que $K \leq|Q(\xi)|, \forall \xi:|\xi| \leq 1$. Então

$$
\frac{K}{\left(1+|\xi|^{2}\right)^{\left|m-\mu^{*}\right|}} \leq K \leq|Q(\xi)|, \forall \xi:|\xi| \leq 1 .
$$

Basta definir então $C=\min \left\{K, C^{*}\right\}$ e $a=\left|m-\mu^{*}\right|$. 


\section{Capítulo 6}

\section{Teorema da Extensão de Whitney}

Nesse capítulo $A \subseteq \mathbb{R}^{n}$ será um fechado, $\alpha$ denotará um multi-índice, isto é, $\alpha \in \mathbb{N}^{n}$, onde $|\alpha|=\alpha_{1}+\ldots+\alpha_{n}$. Denotaremos $D^{\alpha}=\frac{\partial}{\partial \xi_{1}^{\alpha_{1}}}, \ldots, \frac{\partial}{\partial \xi_{n}^{\alpha_{n}}}$.

Whitney encontrou condições necessárias e suficientes na classe das funções $f^{\alpha}$ definidas em $A,|\alpha| \leq m$, para que exista uma função $g \in C^{m}\left(\mathbb{R}^{n}\right)$ que satisfaça

$$
D^{\alpha} g(\xi)=f^{\alpha}(\xi), \forall \xi \in A, \forall \alpha \in \mathbb{N}^{n}:|\alpha| \leq m .
$$

Utilizando o método da extensão de Whitney provaremos que dada uma função $f \in C^{m}\left(\mathbb{R}^{n}\right)$, existe uma função $g \in C^{m}\left(\mathbb{R}^{n}\right)$ tal que

$$
D^{\alpha} g(\xi)=D^{\alpha} f(\xi), \forall \xi \in A, \forall \alpha \in \mathbb{N}^{n}:|\alpha| \leq m,
$$

e tal que é possível estimar o valor das derivadas de $g$ de ordem menor ou igual a $m$ em todo $\mathbb{R}^{n}$ em função dos valores das derivadas de $f$ de ordem menor ou igual a $m$ em $A$.

Teorema 6.0.14 Existe uma seqüência de funções $\left(\varphi_{j}\right)_{j \in \mathbb{N}} \in C_{c}^{\infty}\left(A^{c}\right)$ tal que:

1. $\varphi_{j}(\xi) \geq 0 e \sum_{j} \varphi_{j}(\xi)=1, \forall \xi \notin A$.

2. Todo compacto contido em $A^{c}$ intercepta somente um número finito de suportes das funções $\varphi_{j}$

3. Para todo $\alpha \in \mathbb{N}^{n}$, existe uma constante $C_{\alpha}$ tal que

$$
\sum_{j}\left|D^{\alpha} \varphi_{j}(\xi)\right| \leq C_{\alpha}\left(\operatorname{dist}(\xi, A)^{-|\alpha|}+1\right), \forall \xi \notin A .
$$

4. Existe uma constante $C$ independente de $j$ e de $A$ tal que

$$
\operatorname{diam}\left(\operatorname{supp}\left(\varphi_{j}\right)\right) \leq C \operatorname{dist}\left(\operatorname{supp}\left(\varphi_{j}\right), A\right) .
$$

Para provar esse teorema lançaremos mão de mais alguns resultados.

Definição 6.0.8 Diremos que $Q \subseteq \mathbb{R}^{n}$ é um cubo se $Q$ for um cubo fechado com lados paralelos aos eixos. Dois cubos são ditos disjuntos se seus interiores foram disjuntos. Além disso, se $Q$ é um cubo, denotaremos por $\operatorname{diam}(Q)$ o seu diâmetro. 
Lema 6.0.12 Existe uma seqüência de cubos $\mathcal{F}=\left\{Q_{1}, \ldots, Q_{k}, \ldots\right\}$ tal que:

1. $\cup_{i} Q_{i}=A^{c}$.

2. Os cubos $Q_{k}$ são mutualmente disjuntos.

3. $\operatorname{diam}\left(Q_{k}\right) \leq \operatorname{dist}\left(Q_{k}, A\right) \leq 4 \operatorname{diam}\left(Q_{k}\right), \forall k \in \mathbb{N}$.

Demonstração: Consideremos o reticulado de pontos de $\mathbb{R}^{n}$ cujas coordenadas são inteiras. Tomemos a coleção de cubos cujos lados têm comprimento 1 e cujos vértices pertencem ao reticulado. Chamemos tal coleção de $\mu_{0}$. Por indução formamos novas coleções $\mu_{k}$, de tal forma que cada cubo em $\mu_{k}$ dá origem a $2^{n}$ cubos em $\mu_{k+1}$ por meio da divisão de seus lados em duas partes iguais.

Os cubos de $\mu_{k}$ têm lados de comprimento $\frac{1}{2^{k}}$, e portanto seus diâmetros têm comprimento $\frac{\sqrt{n}}{2^{k}}$. Seja

$$
\Omega_{k}=\left\{x \in \mathbb{R}^{n}: \frac{c}{2^{k}}<\operatorname{dist}(x, A) \leq \frac{c}{2^{k-1}}\right\},
$$

onde $c>0$. É claro que $A^{c}=\cup_{k=-\infty}^{\infty} \Omega_{k}$.

Seja

$$
\mathcal{F}_{0}=\cup_{k \in \mathbb{Z}}\left\{Q \in \mu_{k}: Q \cap \Omega_{k} \neq \emptyset\right\} .
$$

Vejamos que

$$
A^{c} \subseteq \cup_{Q \in \mathcal{F}_{0}} Q .
$$

De fato, se $x \in A^{c}$, então existe um $k \in \mathbb{Z}$ tal que $x \in \Omega_{k}$. Como $\cup_{Q \in \mu_{k}} Q=\mathbb{R}^{n}$, então existe $Q \in \mu_{k}$ tal que $x \in Q$, donde $x \in Q \in \mathcal{F}_{0}$.

Vejamos agora que para uma escolha apropriada de $c$,

$$
\operatorname{diam}(Q) \leq \operatorname{dist}(Q, A) \leq 4 \operatorname{diam}(Q), \forall Q \in \mathcal{F}_{0}
$$

Dado $Q \in \mathcal{F}_{0}$, suponhamos que $Q \in \mu_{k}$. Sabemos que o diâmetro de $Q$ é $\frac{\sqrt{n}}{2^{k}}$ e que existe $x \in Q \cap \Omega_{k}$. Pela equação (6.0.1) podemos dizer que

$$
\operatorname{dist}(Q, A) \leq \operatorname{dist}(x, A) \leq \frac{c}{2^{k-1}} .
$$

Vejamos agora que

$$
\operatorname{dist}(x, A) \leq \operatorname{diam}(Q)+\operatorname{dist}(Q, A) .
$$

Para provar isso, basta ver que $\operatorname{dist}(x, A) \leq \operatorname{diam}(Q)+\operatorname{dist}(y, A), \forall y \in Q$. Como a função $z \longmapsto \operatorname{dist}(z, A)$ é Lipschitziana, então $\operatorname{dist}(x, A)-\operatorname{dist}(y, A) \leq \operatorname{dist}(x, y) \leq \operatorname{diam}(Q)$, pois $x, y \in Q$.

Assim,

$$
\operatorname{dist}(Q, A) \geq d(x, A)-\operatorname{diam}(Q) \geq \frac{c}{2^{k}}-\frac{\sqrt{n}}{2^{k}} .
$$

Seja $c=2 \sqrt{n}$. Obteremos por $(6.0 .2)$ e (6.0.3) que 


$$
\begin{aligned}
\operatorname{diam}(Q) & =\frac{\sqrt{n}}{2^{k}} \\
& \leq \operatorname{dist}(x, A)-\operatorname{diam}(Q) \\
& \leq \operatorname{dist}(Q, A) \\
& \leq \frac{\sqrt{n}}{2^{k-2}} \\
& =4 \operatorname{diam}(Q) .
\end{aligned}
$$

Concluimos que

$$
\operatorname{diam}(Q) \leq \operatorname{dist}(Q, A) \leq 4 \operatorname{diam}(Q) .
$$

A equação (6.0.4) nos permite concluir que os cubos de $\mathcal{F}_{0}$ não interceptam $A$ e que vale o item 3 do lema 6.0.12. Como tínhamos que $A^{c} \subseteq \cup_{Q \in \mathcal{F}_{0}} Q$, podemos concluir que vale o item 1 também. Resta retirar alguns cubos de $\mathcal{F}_{0}$ para obtermos cubos disjuntos.

Vale observar o seguinte fato: se $Q_{1} \in \mu_{k_{1}}$ e $Q_{2} \in \mu_{k_{2}}$ são tais que $Q_{1}$ e $Q_{2}$ não são disjuntos (isto é, seus interiores não são disjuntos) e $k_{2} \leq k_{1}$, então $Q_{1} \subseteq Q_{2}$.

Tomemos agora $Q \in \mathcal{F}_{0}$ e consideremos um cubo maximal em $\mathcal{F}_{0}$ que contém $Q$. A primeira observação a ser feita é que realmente podemos falar em um cubo maximal: digamos que $C, D \in \mathcal{F}_{0}$ e $Q \subseteq C \cap D$. Como $C$ e $D$ não são disjuntos, pela observação acima podemos afirmar que ou $C \subseteq D$ ou vice-versa, de modo que cada cubo em $\mathcal{F}_{0}$ tem um único cubo maximal que o contém (os cubos de $\mathcal{F}_{0}$ que contém $Q$ não podem ser arbitrariamente grandes, uma vez que eles não interceptam $A$ ). Por (6.0.4), se $C \in \mathcal{F}_{0}$ contém $Q$, então

$$
\begin{gathered}
\operatorname{diam}(Q) \leq \operatorname{dist}(Q, A) \leq 4 \operatorname{diam}(Q), \\
\operatorname{diam}(C) \leq \operatorname{dist}(C, A) \leq d(Q, A) \leq 4 \operatorname{diam}(Q),
\end{gathered}
$$

donde $\operatorname{diam}(C) \leq 4 \operatorname{diam}(Q)$. Seja $\mathcal{F}$ a coleção de tais cubos maximais que pertencem a a $\mathcal{F}_{0}$. Os cubos de $\mathcal{F}$ são disjuntos (portanto vale o item 2 ) e $\operatorname{diam}(G) \leq \operatorname{dist}(G, A) \leq 4 \operatorname{diam}(G), \forall G \in \mathcal{F}$ (vale o item 3). Como $\cup_{Q \in \mathcal{F}_{0}} Q=A^{c}$ e os cubos de $\mathcal{F}$ são maximais, essa propriedade é preservada e obtemos o item 1.

Definição 6.0.9 Diremos que dois cubos distintos $Q_{1}$ e $Q_{2}$ de $\mathcal{F}$ se tocam se suas fronteiras tiverem ao menos um ponto em comum.

Proposição 6.0.1 Sejam $Q_{1}$ e $Q_{2}$ elementos de $\mathcal{F}$ que se tocam. Então

$$
\frac{1}{4} \operatorname{diam}\left(Q_{2}\right) \leq \operatorname{diam}\left(Q_{1}\right) \leq 4 \operatorname{diam}\left(Q_{2}\right) .
$$

Demonstração: Seja $p \in Q_{1} \cap Q_{2}$. Temos que dado $q \in Q_{1}$,

$$
\operatorname{dist}(p, A) \leq \operatorname{dist}(p, a) \leq \operatorname{dist}(p, q)+\operatorname{dist}(q, a), \forall a \in A .
$$

Dessa forma $\operatorname{dist}(p, A) \leq \operatorname{dist}(p, q)+\operatorname{dist}(q, A)$. Podemos concluir então que

$$
d\left(Q_{2}, A\right) \leq \operatorname{dist}(p, A) \leq \operatorname{dist}(q, A)+\operatorname{dist}(p, q), \forall q \in Q_{1},
$$


e portanto

$$
\operatorname{dist}\left(Q_{2}, A\right) \leq \operatorname{dist}(q, A)+\operatorname{diam}\left(Q_{1}\right) .
$$

Como isso vale para todo $q \in Q_{1}$, tomando o ínfimo e utilizando o item 3 do lema 6.0.12 concluímos que

$$
\operatorname{dist}\left(Q_{2}, A\right) \leq \operatorname{dist}\left(Q_{1}, A\right)+\operatorname{diam}\left(Q_{1}\right) \leq 4 \operatorname{diam}\left(Q_{1}\right)+\operatorname{diam}\left(Q_{1}\right) .
$$

Pelo item 3 do lema 6.0.12, $\operatorname{diam}\left(Q_{2}\right) \leq \operatorname{dist}\left(Q_{2}, A\right)$. Então $\operatorname{diam}\left(Q_{2}\right) \leq 5 \operatorname{diam}\left(Q_{1}\right)$. Além disso, existe $k \in \mathbb{Z}$ tal que

$$
\operatorname{diam}\left(Q_{1}\right)=2^{k} \operatorname{diam}\left(Q_{2}\right) .
$$

Dessa forma,

$$
\operatorname{diam}\left(Q_{2}\right)=\frac{1}{2^{k}} \operatorname{diam}\left(Q_{1}\right) \leq 5 \operatorname{diam}\left(Q_{1}\right) .
$$

Concluimos que $-2 \leq k$ e, conseqüentemente, $\operatorname{diam}\left(Q_{1}\right) \geq \frac{1}{4} \operatorname{diam}\left(Q_{2}\right)$. Analogamente provamos que $4 \operatorname{diam}\left(Q_{2}\right) \geq \operatorname{diam}\left(Q_{1}\right)$.

Lema 6.0.13 Existe um número $k_{0}$, independente de $A$, tal que se $Q \in \mathcal{F}$, então existem no máximo $k_{0}$ cubos em $\mathcal{F}$ que interceptam $Q$.

Demonstração: Sejam $Q, C \in \mathcal{F}$ cubos que se tocam. Pela Proposição anterior temos que $\frac{1}{4} \operatorname{diam}(Q) \leq \operatorname{diam}(C) \leq 4 \operatorname{diam}(Q)$, e portanto

$$
\frac{1}{4} \leq \frac{\operatorname{diam}(Q)}{\operatorname{diam}(C)} \leq 4
$$

Se $\operatorname{diam}(Q)=\frac{\sqrt{n}}{2^{p}}$, e $\operatorname{diam}(C)=\frac{\sqrt{n}}{2^{q}}$, então $|p-q| \leq \log _{2} 4=2$. Assim sendo, para um $p$ fixado, podemos ter $q=p, q=p-1, q=p-2, q=p+1$ ou $q=p+2$.

Notemos que valem as seguintes estimativas:

1. Se $K \in \mu_{k}$, existem no máximo $3^{n}$ cubos em $\mu_{k}$ que o interceptam.

2. Existem no máximo $2^{n}$ cubos em $\mu_{k-1}$ que interceptam $K$.

3. O número de cubos em $\mu_{k-2}$ que interceptam $K$ também é no máximo $2^{n}$.

4. O número de cubos de $\mu_{k+1}$ que interceptam $K$ só depende de $n$ (e não de $k$ ).

5. O número de cubos de $\mu_{k+2}$ que interceptam $K$ também só depende de $n$.

Tais relações mostram que de fato existe um número $k_{0}$ de modo que dado um cubo de $\mathcal{F}$, existem no máximo $k_{0}$ cubos de $\mathcal{F}$ que o tocam.

Voltando a nossa prova inicial, denotemos por $Q_{k}$ um cubo qualquer de $\mathcal{F}$, por $x_{k}$ seu centro e por $l_{k}$ o comprimento de seus lados. Então $\operatorname{diam}\left(Q_{k}\right)=\sqrt{n} l_{k}$. Seja $\varepsilon$ tal que $0<\varepsilon<\frac{1}{4}$ (que manteremos fixo). Denotemos por $Q_{k}^{*}$ o cubo de mesmo centro que $Q_{k}$ mas expandido pelo fator $1+\varepsilon$, isto é, $Q_{k}^{*}=(1+\varepsilon)\left[Q_{k}-x_{k}\right]+x_{k}$. Temos que $Q_{k} \subseteq Q_{k}^{*}$, mas os cubos $Q_{k}^{*}$ podem não ter interiores disjuntos.

Proposição 6.0.2 Cada ponto de $A^{c}$ está contido em no máximo $k_{0}$ cubos $Q_{j}^{*}$, onde $Q_{j} \in \mathcal{F}$. 
Demonstração: Sejam $Q, Q_{j} \in \mathcal{F}$. Afirmamos que $Q_{j}^{*} \cap Q \neq \emptyset$ se, e somente se, $Q_{j}$ toca $Q$. É claro que se $Q_{j}$ toca $Q$, então $Q_{j}^{*}$ e $Q$ têm intersecção não vazia. Quanto à recíproca, consideremos a união de $Q_{j}$ com todos os cubos de $\mathcal{F}$ que tocam $Q_{j}$. Como esses cubos tocam $Q_{j}$, segue da Proposição 6.0.1 que seus diâmetros são maiores ou iguais a $\frac{1}{4} \operatorname{diam}\left(Q_{j}\right)$; dessa forma o interior de tal união contém $Q_{j}^{*}$ (vale notar que dado um ponto na fronteira de $Q_{j}$, há ao menos mais um cubo de $\mathcal{F}$ que o contém).

Assim, suponhamos que exista $x \in Q \cap Q_{j}^{*}$ mas $Q$ não toca $Q_{j}$. Sabemos que existe um cubo $C$ que toca $Q_{j}$ tal que $x \in C^{\circ}$, uma vez que $x \in Q_{j}^{*}$. Dessa forma obteríamos dois cubos distintos $(Q$ e $C)$ de $\mathcal{F}$ cujos interiores não são disjuntos, o que não é possível.

Dado $x \in A^{c}$, existe um cubo $Q \in \mathcal{F}$ que o contém. Pelo lema 6.0.13 e pelo observado acima existem no máximo $k_{0}$ cubos $Q_{j}^{*}$ que contém $x$.

Lema 6.0.14 Dado $Q_{k} \in \mathcal{F}$,

$$
\operatorname{dist}\left(Q_{k}^{*}, A\right) \leq 4 \operatorname{diam} Q_{k}^{*}
$$

e existe uma constante $C$ independente de $k$ e de $A$ tal que

$$
\operatorname{diam} Q_{k}^{*} \leq C \operatorname{dist}\left(Q_{k}^{*}, A\right) .
$$

Demonstração: Suponhamos que $Q_{k} \in \mu_{k}$. Seja $x \in Q_{k} \cap \Omega_{k}$ (temos que $x \in Q_{k}^{*}$ ). Pela definição de $\Omega_{k}$ obtemos que

$$
\begin{aligned}
\operatorname{dist}\left(Q_{k}^{*}, A\right) & \leq \operatorname{dist}(x, A) \\
& \leq \frac{2 \sqrt{n}}{2^{k-1}}=4 \frac{\sqrt{n}}{2^{k}} \\
& \leq 4 \operatorname{diam} Q_{k}^{*} .
\end{aligned}
$$

Sabemos que

$$
\begin{aligned}
\operatorname{diam} Q_{k}^{*} & =\frac{\sqrt{n}(\varepsilon+1)}{2^{k}} \\
& \leq \frac{5 \sqrt{n}}{4.2^{k}} .
\end{aligned}
$$

Além disso, sejam $q \in Q_{k}, q^{*} \in Q_{k}^{*}$ tais que $\operatorname{dist}(q, A)=\operatorname{dist}\left(Q_{k}, A\right), \operatorname{dist}\left(q^{*}, A\right)=\operatorname{dist}\left(Q_{k}^{*}, A\right)$. Então

$$
\begin{aligned}
\operatorname{dist}\left(Q_{k}, A\right) & =\operatorname{dist}(q, A) \\
& =\operatorname{dist}(q, A)+\operatorname{dist}\left(q^{*}, A\right)-\operatorname{dist}\left(q^{*}, A\right) \\
& \leq \operatorname{dist}\left(Q_{k}^{*}, A\right)+\operatorname{dist}\left(q, q^{*}\right) \\
& \leq \operatorname{dist}\left(Q_{k}^{*}, A\right)+\sqrt{n} \frac{\varepsilon}{2.2^{k}} .
\end{aligned}
$$

Com essa informação concluiremos o lema:

$$
\begin{aligned}
\operatorname{dist}\left(Q_{k}^{*}, A\right) & \geq \operatorname{dist}\left(Q_{k}, A\right)-\sqrt{n} \frac{\varepsilon}{2.2^{k}} \\
& \geq \operatorname{diam} Q_{k}-\frac{\sqrt{n}}{8.2^{k}} \\
& =\frac{\sqrt{n}}{2^{k}}-\frac{\sqrt{n}}{8.2^{k}} \\
& =\frac{7}{8} \frac{\sqrt{n}}{2^{k}} .
\end{aligned}
$$


Assim, basta definir $C=\frac{10}{7}$.

Seja $Q_{0}$ o cubo de centro na origem cujos lados têm comprimento unitário. Fixemos uma função $\psi \in C_{c}^{\infty}$ com as seguintes propriedades:

1. $0 \leq \psi \leq 1$.

2. $\psi(x)=1, \forall x \in Q_{0}$.

3. $\psi(x)=0, \forall x \notin(1+\varepsilon) Q_{0}$.

Para cada $k \in \mathbb{N}$, seja $\psi_{k}$ a função dada por:

$$
\psi_{k}(x)=\psi\left(\frac{x-x_{k}}{l_{k}}\right)
$$

Temos que $\psi_{k}(x)=1$, para todo $x \in Q_{k}$ e $\psi_{k}(x)=0$, se $x \notin Q_{k}^{*}$. Além disso, para todo $\alpha \in \mathbb{N}^{n}$

$$
\left(D^{\alpha} \psi_{k}\right)(x)=\frac{1}{l_{k}^{|\alpha|}}\left(D^{\alpha} \psi\right)\left(\frac{x-x_{k}}{l_{k}}\right) .
$$

Seja

$$
\Phi(x)=\sum_{k=1}^{\infty} \psi_{k}(x), \forall x \in A^{c} .
$$

$\Phi$ está bem definida, pois para cada $x \in A^{c}$ existem no máximo $k_{0}$ cubos $Q_{j}^{*}$ tais que $x \in Q_{j}^{*}$. Além disso, sempre existe algum cubo $Q_{j}$ que contém $x$, donde $1 \leq \Phi(x) \leq k_{0}, \forall x \in A^{c}$. Para $k \in \mathbb{N}$, seja

$$
\varphi_{k}(x)=\frac{\psi_{k}(x)}{\Phi(x)}, \forall x \in A^{c}
$$

É claro que $\varphi_{k} \in C^{\infty}\left(A^{c}\right)$. Além disso, $\operatorname{supp} \varphi_{k} \subseteq Q_{k}^{*}$, e portanto é um compacto. Temos ainda que $\varphi_{k}(x) \geq 0, \forall x \in A^{c}$ e que

$$
\sum_{k=1}^{\infty} \varphi_{k}(x)=1, \forall x \in A^{c} .
$$

Lema 6.0.15 Para cada $\alpha \in \mathbb{N}^{n}$ existe uma constante $C_{\alpha}$ tal que

$$
\left|\left(D^{\alpha} \Phi\right)(x)\right| \leq \frac{C_{\alpha}}{\operatorname{dist}(x, A)^{|\alpha|}}, \forall x \in A^{c} .
$$

Demonstração: Dado $x \in A^{c}$, existe um conjunto finito $J_{x} \subseteq \mathbb{N}$ tal que se $k \notin J_{x}$, então $x \notin Q_{k}^{*}$. Adicionalmente, sabemos que o número de elementos de $J_{x}$ é no máximo $k_{0}, \forall x \in A^{c}$. Assim sendo,

$$
\sum_{k=1}^{\infty}\left(D^{\alpha} \psi_{k}\right)(x)=\sum_{k \in J_{x}} \frac{1}{l_{k}^{|\alpha|}} D^{\alpha} \psi\left(\frac{x-x_{k}}{l_{k}}\right) .
$$


Dessa forma,

$$
\begin{aligned}
\left|D^{\alpha} \Phi(x)\right| & \leq\left\|D^{\alpha} \psi\right\|_{\infty} \sum_{k \in J_{x}} \frac{1}{l_{k}^{|\alpha|}} \\
& \leq k_{0}\left\|D^{\alpha} \psi\right\|_{\infty} \sup _{k \in J_{x}} \frac{1}{l_{k}^{|\alpha|}} .
\end{aligned}
$$

Seja $y \in Q_{k}^{*}$ tal que $\operatorname{dist}(y, A)=\operatorname{dist}\left(Q_{k}^{*}, A\right)$. Pelo lema 6.0.14 podemos afirmar que $\operatorname{dist}(y, A) \leq 4 \operatorname{diam}\left(Q_{k}^{*}\right)$. Segue que para todo $x \in Q_{k}^{*}$,

$$
\begin{aligned}
\operatorname{dist}(x, A) & =\operatorname{dist}(x, A)-\operatorname{dist}(y, A)+\operatorname{dist}(y, A) \\
& \leq \operatorname{dist}(x, A)-\operatorname{dist}(y, A)+4 \operatorname{diam}\left(Q_{k}^{*}\right) \\
& \leq \operatorname{dist}(x, y)+4 \operatorname{diam}\left(Q_{k}^{*}\right) \\
& \leq 5 \operatorname{diam}\left(Q_{k}^{*}\right) \\
& =5 \sqrt{n} l_{k}(1+\varepsilon) .
\end{aligned}
$$

Concluímos que para todo $x \in Q_{k}^{*}$,

$$
\frac{1}{l_{k}} \leq \frac{5 \sqrt{n}(1+\varepsilon)}{\operatorname{dist}(x, A)}
$$

e portanto

$$
\begin{aligned}
\left|\left(D^{\alpha} \Phi\right)(x)\right| & \leq k_{0}|| D^{\alpha} \psi \|_{\infty}\left(\frac{5 \sqrt{n}(1+\varepsilon)}{\operatorname{dist}(x, A)}\right)^{|\alpha|} \\
& =\frac{C_{\alpha}}{\operatorname{dist}(x, A)^{|\alpha|}} .
\end{aligned}
$$

Lema 6.0.16 Para todo $\alpha \in \mathbb{N}^{n}$ existe uma constante $M_{\alpha}$ tal que

$$
\left|\left(D^{\alpha} \frac{1}{\Phi}\right)(x)\right| \leq M_{\alpha} \frac{1}{\operatorname{dist}(x, A)^{|\alpha|}}, \forall x \in A^{c} .
$$

Demonstração: Faremos a prova por indução em $|\alpha|$. A afirmação é imediata se $|\alpha|=0$. Suponhamos que o resultado é verdadeiro se $|\alpha|=l-1$, onde $l \geq 1$, e provemos que vale se $|\alpha|=l$. Seja $\alpha \in \mathbb{N}^{n}$ tal que $|\alpha|=l$.

$$
\begin{aligned}
0 & =D^{\alpha}\left(\Phi \frac{1}{\Phi}\right)(x) \\
& =\sum_{\beta \leq \alpha}\left(\begin{array}{c}
\alpha \\
\beta
\end{array}\right) D^{\alpha-\beta} \frac{1}{\Phi}(x) D^{\beta} \Phi(x) \\
& =\Phi(x) D^{\alpha} \frac{1}{\Phi}(x)+\sum_{0<\beta \leq \alpha}\left(\begin{array}{l}
\alpha \\
\beta
\end{array}\right) D^{\alpha-\beta} \frac{1}{\Phi}(x) D^{\beta} \Phi(x) .
\end{aligned}
$$

Assim,

$$
D^{\alpha} \frac{1}{\Phi}(x)=-\left[\sum_{0<\beta \leq \alpha}\left(\begin{array}{l}
\alpha \\
\beta
\end{array}\right) D^{\alpha-\beta} \frac{1}{\Phi}(x) D^{\beta} \Phi(x)\right] \frac{1}{\Phi(x)} .
$$


Utilizando primeiramente a equação (6.0.5) e depois a hipótese de indução obtemos:

$$
\begin{aligned}
\left|D^{\alpha} \frac{1}{\Phi(x)}\right| & \leq \sum_{0<\beta \leq \alpha}\left(\begin{array}{c}
\alpha \\
\beta
\end{array}\right)\left|D^{\alpha-\beta} \frac{1}{\Phi}(x)\right| \frac{C_{\beta}}{\operatorname{dist}(x, A)^{|\beta|}} \\
& \leq \sum_{0<\beta \leq \alpha}\left(\begin{array}{c}
\alpha \\
\beta
\end{array}\right) \frac{M_{\alpha-\beta}}{\operatorname{dist}(x, A)^{|\alpha-\beta|}} \frac{C_{\beta}}{\operatorname{dist}(x, A)^{|\beta|}} \\
& \leq M_{\alpha} \operatorname{dist}(x, A)^{-|\alpha|} .
\end{aligned}
$$

Lema 6.0.17 Dado $\alpha \in \mathbb{N}^{n}$, existe uma constante $E_{\alpha}$ tal que para todo $k \in \mathbb{N}$,

$$
\left|D^{\alpha}\left(\frac{\psi_{k}}{\Phi}\right)(x)\right| \leq E_{\alpha}\left(\operatorname{dist}(x, A)^{-|\alpha|}+1\right), \forall x \in Q_{k}^{*} .
$$

Demonstração: Sejam $\alpha \in \mathbb{N}^{n}, k \in \mathbb{N}$ e $x \in Q_{k}^{*}$. Temos que

$$
\begin{aligned}
\left|D^{\alpha}\left(\frac{\psi_{k}}{\Phi}\right)(x)\right| & \leq C \sum_{\beta \leq \alpha}\left|\left(D^{\beta} \frac{1}{\Phi}\right)(x) D^{\alpha-\beta} \psi_{k}(x)\right| \\
& \leq C \sum_{0<\beta \leq \alpha} \frac{M_{\beta}}{\operatorname{dist}(x, A)^{|\beta|}}\left|D^{\alpha-\beta} \psi_{k}(x)\right|+C\left|\frac{D^{\alpha} \psi_{k}(x)}{\Phi(x)}\right| \\
& \leq C \sum_{0<\beta \leq \alpha} \frac{M_{\beta}}{\operatorname{dist}(x, A)^{|\beta|}}|| D^{\alpha-\beta} \psi_{k}\left\|_{\infty}+C|| D^{\alpha} \psi_{k}\right\|_{\infty} \\
& \leq C \sum_{0<\beta \leq \alpha} \frac{M_{\beta}}{\operatorname{dist}(x, A)^{|\beta|}} \frac{1}{|\alpha-\beta|}|| D^{\alpha-\beta} \psi\left\|_{\infty}+\frac{C}{l_{k}^{|\alpha|}}\right\| D^{\alpha} \psi \|_{\infty} \\
& \leq C \sum_{0<\beta \leq \alpha} \frac{M_{\beta}}{\operatorname{dist}(x, A)^{|\beta|} \mid} \mid D^{\alpha-\beta} \psi \|_{\infty}\left(\frac{5 \sqrt{n}(1+\varepsilon)}{\operatorname{dist}(x, A)}\right)^{|\alpha-\beta|}+ \\
& +C\left(\frac{5 \sqrt{n}(1+\varepsilon)}{\operatorname{dist}(x, A)}\right)^{|\alpha|}\left\|D^{\alpha} \psi\right\|_{\infty} \\
& \leq E_{\alpha} \operatorname{dist}(x, A)^{-|\alpha|} .
\end{aligned}
$$

Lema 6.0.18 Dado $\alpha \in \mathbb{N}^{n}$, existe uma constante $C_{\alpha}$ tal que

$$
\sum_{k=1}^{\infty}\left|D^{\alpha} \varphi_{k}(x)\right| \leq C_{\alpha}\left(\operatorname{dist}(x, A)^{-|\alpha|}+1\right), \forall x \in A^{c} .
$$

Demonstração: Seja $x \in A^{c}$. Utilizaremos o lema anterior.

$$
\begin{aligned}
\sum_{k=1}^{\infty}\left|D^{\alpha} \varphi_{k}(x)\right| & =\sum_{k \in J_{x}}\left|D^{\alpha} \varphi_{k}(x)\right| \\
& \leq \sum_{k \in J_{x}} E_{\alpha}\left(\operatorname{dist}(x, A)^{-|\alpha|}+1\right) \\
& \leq k_{0} E_{\alpha}\left(\operatorname{dist}(x, A)^{-|\alpha|}+1\right) .
\end{aligned}
$$


Lema 6.0.19 Se $K \subseteq A^{c}$ é um compacto, $K$ intercepta somente um número finitos de suportes das funções $\varphi_{k}$.

Demonstração: Como supp $\varphi_{k} \subseteq Q_{k}^{*}, \forall k \in \mathbb{N}$, é suficiente mostrarmos que $K$ intercepta somente um número finito de cubos $Q_{k}^{*}$. Como $K \subseteq A^{c}$ é compacto e $\left\{\left(Q_{k}^{*}\right)^{\circ}\right\}_{k \in \mathbb{N}}$ é uma cobertura aberta de $A^{c}$, existem $i_{1}, \ldots, i_{l}$ tais que

$$
K \subseteq\left(Q_{i_{1}}^{*}\right)^{\circ} \cup \ldots \cup\left(Q_{i_{l}}^{*}\right)^{\circ}
$$

Dado $k \in \mathbb{N}, K \cap Q_{k}^{*} \neq \emptyset$ se, e somente se, existir $j \leq l$ tal que $Q_{k}^{*} \cap Q_{i_{j}}^{*} \neq \emptyset$. Como o número máximo de $k \in \mathbb{N}$ que satisfazem isso é $k_{0}$, segue que $K$ intercepta no máximo $k_{0} . l$ cubos $Q_{k}^{*}$.

Para concluir o teorema 6.0.14 resta provar o seguinte:

Lema 6.0.20 Existe uma constante $C$ que não depende de $j$, nem de $A$, tal que o diâmetro do suporte de $\varphi_{j}$ é menor ou igual a $C$ vezes sua distância até $A$.

Demonstração: Sabemos que $\operatorname{supp} \varphi_{j} \subseteq Q_{j}^{*}$. Pelo lema 6.0.14,

$$
\operatorname{diam}\left(\operatorname{supp} \varphi_{j}\right) \leq \operatorname{diam} Q_{j}^{*} \leq C \operatorname{dist}\left(Q_{j}^{*}, A\right) \leq C \operatorname{dist}\left(\operatorname{supp} \varphi_{j}, A\right) .
$$

A partir de agora continuaremos a trabalhar com as funções $\varphi_{k}$ e com os cubos $Q_{k}^{*}$ para provar que dada uma função $f \in C^{m}\left(\mathbb{R}^{n}\right)$, existe uma função $g \in C^{m}\left(\mathbb{R}^{n}\right)$ tal que

$$
D^{\alpha} g(\xi)=D^{\alpha} f(\xi), \forall \xi \in A, \forall \alpha \in \mathbb{N}^{n}:|\alpha| \leq m,
$$

e tal que é possível estimar o valor das derivadas de $g$ de ordem menor ou igual a $m$ em todo $\mathbb{R}^{n}$ em função dos valores das derivadas de $g$ de ordens menores ou iguais a $m$ em $A$.

Para cada $j$, seja $\xi^{j} \in A$ de tal modo que

$$
\operatorname{dist}\left(\operatorname{supp}\left(\varphi_{j}\right), A\right)=\operatorname{dist}\left(\operatorname{supp}\left(\varphi_{j}\right), \xi^{j}\right),
$$

Note que tal escolha é possível pois $\operatorname{supp} \varphi_{j}$ é compacto e $A$ é fechado.

Dada uma função $f \in \mathcal{C}^{m}\left(\mathbb{R}^{n}\right)$, seja

$$
g(\xi)=\left\{\begin{aligned}
\sum^{*} \varphi_{j}(\xi) f_{m}\left(\xi, \xi^{j}\right), & \text { se } \xi \notin A \\
f(\xi), & \text { se } \xi \in A
\end{aligned}\right.
$$

onde

$$
f_{m}(\xi, \eta)=\sum_{|\alpha| \leq m} D^{\alpha} f(\eta) \frac{(\xi-\eta)^{\alpha}}{\alpha !}
$$

é a expansão de Taylor de $f$ de ordem $m$ em $\eta$ e $\sum^{*}$ indica que estamos somando apenas sobre os $j$ tais que $l_{j}<1$.

Lema 6.0.21 Se $\operatorname{dist}(\xi, A)>\frac{21 \sqrt{n}}{8}$, então $g(\xi)=0$. 
Demonstração: Suponhamos que $\xi \in Q^{*}$, onde $Q \in \mathcal{F}$ e $Q \in \mu_{k}$. Pela construção de $\mathcal{F}$ existe um ponto $y \in Q$ tal que

$$
\frac{\sqrt{n}}{2^{k-1}}<\operatorname{dist}(y, A) \leq \frac{\sqrt{n}}{2^{k-2}} .
$$

Então

$$
\begin{aligned}
\frac{21 \sqrt{n}}{8} & <\operatorname{dist}(\xi, A) \\
& \leq \operatorname{dist}(y, A)+\operatorname{diam}\left(Q^{*}\right) \\
& \leq \frac{\sqrt{n}}{2^{k-2}}+\frac{\sqrt{n}(1+\varepsilon)}{2^{k}} \\
& \leq \frac{16 \sqrt{n}}{4.2^{k}}+\frac{5 \sqrt{n}}{4.2^{k}} \\
& \leq \frac{21 \sqrt{n}}{4.2^{k}} .
\end{aligned}
$$

Assim vem que $1<\frac{1}{2^{k-1}}$, isto é, $k \leq 0$. Assim sendo, $l_{k}=\frac{1}{2^{k}} \geq 1$, donde $g(\xi)=0$.

Lema 6.0.22 Com as definições acima, $g \in \mathcal{C}^{m}\left(\mathbb{R}^{n}\right)$ e $D^{\alpha} g=D^{\alpha} f$ em $A, \forall|\alpha| \leq m$.

Demonstração: Vamos introduzir mais algumas notações. Seja $R_{m}(\xi ; \eta)$ o resto da fórmula de Taylor, isto é,

$$
f(\xi)=f_{m}(\xi, \eta)+R_{m}(\xi ; \eta) .
$$

Diferenciando (sempre com relação a $\xi$ ) obtemos:

$$
f^{(\alpha)}(\xi)=f_{m}^{(\alpha)}(\xi, \eta)+R_{m}^{(\alpha)}(\xi ; \eta)
$$

Fixemos $\xi \notin A$ e seja $\xi^{*} \in A$ tal que

$$
\left|\xi^{*}-\xi\right|=\operatorname{dist}(\xi, A)
$$

Desejamos comparar $g^{(\alpha)}(\xi) \operatorname{com} f_{m}^{(\alpha)}\left(\xi, \xi^{*}\right)$.

Lema 6.0.23 Se $\operatorname{dist}(\xi, A)<\frac{3}{4}$, então $\sum^{*} \varphi_{j}(\xi)=1$.

Demonstração: Suponhamos que $\xi \in Q^{*}$, onde $Q \in \mathcal{F}$ e que $Q \in \mu_{k}$. Seja $y \in Q$ tal que

$$
\frac{\sqrt{n}}{2^{k-1}}<\operatorname{dist}(y, A) \leq \frac{\sqrt{n}}{2^{k-2}} \text {. }
$$

Temos que

$$
\begin{aligned}
\frac{\sqrt{n}}{2^{k-1}} & <\operatorname{dist}(y, A) \leq \operatorname{dist}(\xi, A)+\operatorname{diam}\left(Q^{*}\right) \\
& \leq \frac{3}{4}+\frac{\sqrt{n}(1+\varepsilon)}{2^{k}} \\
& \leq \frac{3}{4}+\frac{5 \sqrt{n}}{4.2^{k}} .
\end{aligned}
$$

Assim, $\frac{1}{2^{k}} \leq \frac{\sqrt{n}}{2^{k}}<1$, isto é, $1<2^{k}$ e, portanto $k>0$. Dessa forma $l_{k}=\frac{1}{2^{k}}<1$ e então pelo teorema 6.0.14,

$$
\sum_{j}^{*} \varphi_{j}(\xi)=\sum_{j} \varphi_{j}(\xi)=1
$$


Conseqüentemente, se $\operatorname{dist}(\xi, A)<\frac{3}{4}$ e $\xi \notin A$, então

$$
g(\xi)=f_{m}\left(\xi, \xi^{*}\right)+\sum_{j}^{*} \varphi_{j}(\xi)\left(f_{m}\left(\xi ; \xi^{j}\right)-f_{m}\left(\xi, \xi^{*}\right)\right)
$$

Diferenciando e utilizando a fórmula de Leibniz teremos:

$$
g^{(\alpha)}(\xi)=f_{m}^{(\alpha)}\left(\xi ; \xi^{*}\right)+\sum_{\beta+\gamma=\alpha}\left(\begin{array}{c}
\alpha \\
\beta
\end{array}\right) \sum_{j}^{*} \varphi_{j}^{(\beta)}(\xi)\left(f_{m}^{(\gamma)}\left(\xi ; \xi^{j}\right)-f_{m}^{(\gamma)}\left(\xi ; \xi^{*}\right)\right) .
$$

Vamos assumir que $\xi \in \operatorname{supp} \varphi_{j}$. Temos que

$$
\operatorname{dist}\left(\operatorname{supp} \varphi_{j}, A\right) \leq \operatorname{dist}\left(\operatorname{supp} \varphi_{j}, \xi^{*}\right) \leq\left|\xi-\xi^{*}\right|,
$$

e, pelo teorema 6.0.14, item 4. existe uma constante $C>0$ (independente de $j$ e de $A$ ) tal que

$$
\operatorname{diam}\left(\operatorname{supp} \varphi_{j}\right) \leq C\left|\xi-\xi^{*}\right|
$$

Seja $y \in \operatorname{supp} \varphi_{j}$ tal que $\operatorname{dist}\left(\operatorname{supp} \varphi_{j}, \xi^{j}\right)=\left|y-\xi^{j}\right|$. Assim,

$$
\begin{aligned}
\left|\xi-\xi^{j}\right| & \leq|\xi-y|+\left|y-\xi^{j}\right| \\
& \leq \operatorname{diam}\left(\operatorname{supp} \varphi_{j}\right)+\left|y-\xi^{j}\right| \\
& =\operatorname{diam}\left(\operatorname{supp} \varphi_{j}\right)+\operatorname{dist}\left(\operatorname{supp} \varphi_{j}, \xi^{j}\right) \\
& =\operatorname{diam}\left(\operatorname{supp} \varphi_{j}\right)+\operatorname{dist}\left(\operatorname{supp} \varphi_{j}, A\right) \\
& \leq C\left|\xi-\xi^{*}\right|+\left|\xi-\xi^{*}\right| \\
& =(C+1) \operatorname{dist}(\xi, A) .
\end{aligned}
$$

Dessa forma,

$$
\begin{aligned}
\left|\xi^{j}-\xi^{*}\right| & \leq\left|\xi^{j}-\xi\right|+\left|\xi-\xi^{*}\right| \\
& \leq(C+1)\left|\xi-\xi^{*}\right|+\left|\xi-\xi^{*}\right| \\
& =(C+2) \operatorname{dist}(\xi, A) .
\end{aligned}
$$

Na equação (6.0.7), substituindo expressões teremos:

$$
g^{(\alpha)}(\xi)=f^{(\alpha)}(\xi)-R_{m}^{(\alpha)}\left(\xi ; \xi^{*}\right)+\sum_{\beta+\gamma=\alpha}\left(\begin{array}{c}
\alpha \\
\beta
\end{array}\right) \sum_{j}^{*} \varphi_{j}^{(\beta)}(\xi)\left(R_{m}^{(\gamma)}\left(\xi ; \xi^{*}\right)-R_{m}^{(\gamma)}\left(\xi ; \xi^{j}\right)\right) .
$$

Lema 6.0.24 Se $\xi, \eta$ são elementos de um compacto fixo, então

$$
\left|R_{m}^{(\gamma)}(\xi ; \eta)\right| \leq|\xi-\eta|^{m-|\gamma|} \varepsilon(\xi, \eta),
$$

onde $\varepsilon(z, w) \stackrel{u}{\rightarrow} 0$ quando $|z-w| \rightarrow 0$.

Demonstração: Digamos que $\xi, \eta \in K$, onde $K$ é compacto. Pela fórmula de Taylor,

$$
f(\xi)=\sum_{|\beta| \leq m-1} D^{\beta} f(\eta) \frac{(\xi-\eta)^{\beta}}{\beta !}+m \int_{0}^{1} \sum_{|\gamma|=m} D^{\gamma} f(\eta+t(\xi-\eta))(\xi-\eta)^{\gamma} \frac{(1-t)^{m-1}}{\gamma !} d t .
$$


Podemos escrever que

$$
\begin{aligned}
R_{m}(\xi ; \eta) & =f(\xi)-f_{m}(\xi, \eta) \\
& =f(\xi)-\sum_{|\beta| \leq m-1} D^{\beta} f(\eta) \frac{(\xi-\eta)^{\beta}}{\beta !}-\sum_{|\beta|=m} D^{\beta} f(\eta) \frac{(\xi-\eta)^{\beta}}{\beta !} \\
& =m \int_{0}^{1} \sum_{|\gamma|=m} D^{\gamma} f(\eta+t(\xi-\eta))(\xi-\eta)^{\gamma} \frac{(1-t)^{m-1}}{\gamma !} d t-\sum_{|\beta|=m} D^{\beta} f(\eta) \frac{(\xi-\eta)^{\beta}}{\beta !} \\
& =m \int_{0}^{1} \sum_{|\gamma|=m}\left(D^{\gamma} f(\eta+t(\xi-\eta))-D^{\gamma} f(\eta)\right) \frac{(\xi-\eta)^{\gamma}}{\gamma !}(1-t)^{m-1} d t .
\end{aligned}
$$

Dessa forma,

$$
\left|R_{m}(\xi ; \eta)\right| \leq m \int_{0}^{1} \sum_{|\gamma|=m}\left|D^{\gamma} f(\eta+t(\xi-\eta))-D^{\gamma} f(\eta)\right|(1-t)^{m-1} \frac{|\xi-\eta|^{m}}{\gamma !} d t
$$

Seja

$$
\varepsilon(\xi, \eta)=m \sum_{|\gamma|=m} \operatorname{supp}_{0 \leq t \leq 1}\left|D^{\gamma} f(\eta+t(\xi-\eta))-D^{\gamma} f(\eta)\right| \frac{(1-t)^{m-1}}{\gamma !}
$$

Vejamos que $\varepsilon(\xi, \eta) \stackrel{u}{\rightarrow} 0$ se $|\xi-\eta| \rightarrow 0$. Para isso basta provarmos que para cada $\gamma$ fixado com $|\gamma|=m$, a função abaixo tende uniformemente a zero quando $|\xi-\eta| \rightarrow 0$ :

$$
\operatorname{supp}_{0 \leq t \leq 1}\left|D^{\gamma} f(\eta+t(\xi-\eta))-D^{\gamma} f(\eta)\right|(1-t)^{m-1}
$$

Como a função $f$ é de classe $\mathcal{C}^{m}$, a seguinte função, $h$, é contínua:

$$
K \times K \times[0,1] \ni(\xi, \eta, t) \mapsto D^{\gamma} f(\eta+t(\xi-\eta))(1-t)^{m-1}
$$

Como $[0,1]$ é compacto, então $K \times K \times[0,1]$ é um compacto e portanto $h$ é uniformemente contínua. Conseqüentemente, dado $\varepsilon>0$ existe $\delta>0$ tal que se $\left|(\xi, \eta, t)-\left(\xi^{\prime}, \eta^{\prime}, t^{\prime}\right)\right|<\delta$, então $\left|h(\xi, \eta, t)-h\left(\xi^{\prime}, \eta^{\prime}, t^{\prime}\right)\right|<\varepsilon$. Sejam então $\xi, \eta \in K:|\xi-\eta|<\delta$. Para todo $t \in[0,1]$, $|(\xi, \eta, t)-(\eta, \eta, t)|=|\xi-\eta|<\delta$, donde

$$
\left|D^{\gamma} f(\eta+t(\xi-\eta))-D^{\gamma} f(\eta)\right|(1-t)^{m-1}<\varepsilon
$$

Para provarmos o caso geral (isto é, $|\gamma| \leq m$ ), utilizamos o argumento acima com $D^{\gamma} f$ no lugar de $f$, notando que teremos então uma função de classe $m-|\gamma|$. 
Sejam $d=\left|\xi-\xi^{*}\right|=\operatorname{dist}(\xi, A)$ e $d_{1}=(C+1) d$. Pelo teorema 6.0.14 item 3 e por (6.0.8),

$$
\begin{aligned}
\left|g^{(\alpha)}(\xi)-f^{(\alpha)}(\xi)\right| \leq\left|R_{m}^{(\alpha)}\left(\xi ; \xi^{*}\right)\right|+\sum_{\beta+\gamma=\alpha}\left(\begin{array}{c}
\alpha \\
\beta
\end{array}\right) \sum_{j}^{*}\left|\varphi_{j}^{(\beta)}(\xi)\right|\left(\left|R_{m}^{(\gamma)}\left(\xi ; \xi^{j}\right)\right|+\left|R_{m}^{(\gamma)}\left(\xi ; \xi^{*}\right)\right|\right) \\
\quad \leq d^{m-|\alpha|} \varepsilon\left(\xi, \xi^{*}\right)+K_{1} \sum_{\beta+\gamma=\alpha} \sum_{j}^{*}\left|\varphi_{j}^{(\beta)}(\xi)\right|\left(\left|R_{m}^{(\gamma)}\left(\xi ; \xi^{j}\right)\right|+\left|R_{m}^{(\gamma)}\left(\xi ; \xi^{*}\right)\right|\right) \\
\quad \leq d^{m-|\alpha|} \varepsilon\left(\xi, \xi^{*}\right)+ \\
+K_{1} \sum_{\beta+\gamma=\alpha} \sum_{j}^{*}\left|\varphi_{j}^{(\beta)}(\xi)\right|\left(\left|\xi-\xi^{j}\right|^{m-|\gamma|} \varepsilon\left(\xi, \xi^{j}\right)+d^{m-|\gamma|} \varepsilon\left(\xi, \xi^{*}\right)\right) \\
\quad \leq d^{m-|\alpha|} \varepsilon\left(\xi, \xi^{*}\right)+K_{1} \sum_{\beta+\gamma=\alpha} \sum_{j}^{*}\left|\varphi_{j}^{(\beta)}(\xi)\right|\left(d_{1}^{m-|\gamma|} \varepsilon\left(\xi, \xi^{j}\right)+d^{m-|\gamma|} \varepsilon\left(\xi, \xi^{*}\right)\right) \\
\leq d^{m-|\alpha|} \varepsilon\left(\xi, \xi^{*}\right)+K_{1} \sum_{\beta+\gamma=\alpha} C_{\beta}\left(d^{-|\beta|}+1\right)\left(d_{1}^{m-|\gamma|} \varepsilon\left(\xi, \xi^{j}\right)+d^{m-|\gamma|} \varepsilon\left(\xi, \xi^{*}\right)\right) .
\end{aligned}
$$

Em resumo:

$$
\left|g^{(\alpha)}(\xi)-f^{(\alpha)}(\xi)\right| \leq d^{m-|\alpha|} \varepsilon\left(\xi, \xi^{*}\right)+K_{1} \sum_{\beta+\gamma=\alpha} C_{\beta}\left(d^{-|\beta|}+1\right)\left(d_{1}^{m-|\gamma|} \varepsilon\left(\xi, \xi^{j}\right)+d^{m-|\gamma|} \varepsilon\left(\xi, \xi^{*}\right)\right) .
$$

Vejamos que (6.0.9) tende a 0 quando $d \rightarrow 0$. Como $|\alpha| \leq m, d^{m-|\alpha|} \varepsilon\left(\xi, \xi^{*}\right) \stackrel{d \rightarrow 0}{\rightarrow} 0$. Além disso, $\left|\xi-\xi^{j}\right| \leq(C+1) \operatorname{dist}(\xi, A)$ e portanto $\varepsilon\left(\xi, \xi^{j}\right) \rightarrow 0$ quando $d \rightarrow 0$. Finalmente, $-|\beta|+m-|\gamma|=m-|\alpha| \geq 0$ e portanto vale a afirmação acima.

Seja $h=f-g$. Temos que $h=0$ em $A$ e que $h \in \mathcal{C}^{m}\left(A^{c}\right)$; além disso, suas derivadas de ordem menor ou igual a $m$ tendem a 0 se $\operatorname{dist}(\xi, A) \rightarrow 0$. Isso será suficiente para provar que $h \in \mathcal{C}^{m}\left(\mathbb{R}^{n}\right)$ e que suas derivadas de ordem menor ou igual a $m$ se anulam em $A$ (com isso concluiremos o lema).

Basta provarmos que as derivadas parciais de $h$ existem e se anulam nos pontos da fronteira de A.

Lema 6.0.25 Sejam I um intervalo fechado, $F \subseteq I$ um fechado. Seja $f$ uma função contínua definida em I tal que $f$ é diferenciável fora de $F$ e $f$ se anula em $F$. Se para todo $x \in F$, $f^{\prime}(y) \rightarrow 0$ quando $y \rightarrow x, y \in I \cap F^{c}$, então $f^{\prime}(x)$ existe e é igual a 0.

Demonstração: Fixado $x \in F$, queremos estudar o seguinte limite:

$$
\lim _{y \rightarrow x} \frac{f(y)-f(x)}{y-x} .
$$

Se $y \in F$, então $f(y)=0$, donde $|f(y)-f(x)|=0$. Se $y \notin F$, seja $z \in F \cap[x, y]$ o ponto mais próximo de $y$ (esse ponto existe pois $F \cap[x, y]$ é um fechado). Lembrando que $x, z \in F$ e aplicando a Desigualdade do Valor Médio, temos:

$$
\begin{aligned}
|f(y)-f(x)| & =|f(y)-f(z)| \\
& \leq|y-z| \sup _{0<t<1}\left|f^{\prime}(z+t(y-z))\right| \\
& \leq|x-y| \sup _{0<t<1}\left|f^{\prime}(z+t(y-z))\right| .
\end{aligned}
$$


Como $f^{\prime}(y) \rightarrow 0$ quando $y \rightarrow x$, então $f$ é derivável em $x$ e $f^{\prime}(x)=0$.

Isso conclui a prova do lema 6.0.22

Definição 6.0.10 Se $B \subseteq \mathbb{R}^{n}$ e $f \in \mathcal{C}^{m}\left(\mathbb{R}^{n}\right)$, denotamos por $|f|_{m, B}$ o supremo dos valores:

$$
\begin{gathered}
\left|f^{(\alpha)}(\xi)\right|,|\alpha| \leq m, \xi \in B, \\
\frac{\left|R_{m}^{(\alpha)}(\xi ; \eta)\right|}{|\xi-\eta|^{m-|\alpha|}},|\alpha| \leq m, \xi, \eta \in B, \xi \neq \eta .
\end{gathered}
$$

Se $B$ for vazio, definimos $|f|_{m, B}=0$ e se $B=\{\xi\}$ escrevemos $|f|_{m, \xi}$, isto é,

$$
|f|_{m, \xi}=\sup \left\{\left|f^{(\alpha)}(\xi)\right|:|\alpha| \leq m\right\}
$$

Vamos definir também

$$
A_{\xi}=\left\{x \in A:|x-\xi| \leq R=(C+1) \frac{21 \sqrt{n}}{8}\right\}
$$

Nosso objetivo é provar que existe uma constante $K$ independente de $f$ e de $\xi$ tal que

$$
|g|_{m, \xi} \leq K|f|_{m, A_{\xi}}, \forall \xi \in \mathbb{R}^{n}
$$

Notemos inicialmente que essa desigualdade é imediata se $\operatorname{dist}(\xi, A)>\frac{21 \sqrt{n}}{8}$. De fato, vimos que existe uma vizinhança $U$ de $\xi$ na qual $g=0$, e portanto o lado esquerdo da igualdade é 0 .

Se $\operatorname{dist}(\xi, A) \leq \frac{21 \sqrt{n}}{8}$, então $\xi^{*} \in A_{\xi}$ pois escolhemos $\xi^{*} \in A$ de modo que $\left|\xi^{*}-\xi\right|=\operatorname{dist}(\xi, A)$. Se $\xi \in \operatorname{supp} \varphi_{j}$, então $\xi^{j} \in A_{\xi}$ pois

$$
\left|\xi-\xi^{j}\right| \leq(C+1) \operatorname{dist}(\xi, A) \leq R
$$

Vamos supor inicialmente que

$$
\frac{3}{4} \leq \operatorname{dist}(\xi, A) \leq \frac{21 \sqrt{n}}{8}
$$

Não podemos usar (6.0.6), mas

$$
g^{(\alpha)}(\xi)=\sum_{j}^{*} \sum_{\beta+\gamma=\alpha}\left(\begin{array}{l}
\alpha \\
\beta
\end{array}\right) \varphi_{j}^{(\beta)}(\xi) f_{m}^{(\gamma)}\left(\xi, \xi^{j}\right) .
$$

Se $\gamma$ não é menor ou igual a $\alpha$, então $f_{m}^{(\gamma)}\left(\xi, \xi^{j}\right)=0$, mas se $\gamma \leq \alpha$,

$$
\begin{aligned}
f_{m}^{(\gamma)}\left(\xi, \xi^{j}\right) & =\sum_{|\alpha| \leq m} f^{(\alpha)}\left(\xi^{j}\right) \frac{\left(\xi-\xi^{j}\right)^{\alpha-\gamma}}{\alpha !} \frac{\alpha !}{(\alpha-\gamma) !} \\
& =\sum_{|\beta| \leq m-|\gamma|} f^{(\gamma+\beta)}\left(\xi^{j}\right) \frac{\left(\xi-\xi^{j}\right)^{\beta}}{\beta !}
\end{aligned}
$$


Se $\xi \in \operatorname{supp} \varphi_{j}$ e $\varphi_{j}^{(\beta)}(\xi) \neq 0$, queremos estimar $f_{m}^{(\gamma)}\left(\xi, \xi^{j}\right)$ por $|f|_{m, A_{\xi}}$. Isso será possível pois $\xi^{j} \in A_{\xi}$ e $\left|\xi-\xi^{j}\right| \leq R$. De fato,

$$
\begin{aligned}
\left|f_{m}^{(\gamma)}\left(\xi, \xi^{j}\right)\right| & \leq \sum_{|\beta| \leq m-|\gamma|}\left|f^{(\gamma+\beta)}\left(\xi^{j}\right)\right| \frac{\left|\xi-\xi^{j}\right| \beta \mid}{\beta !} \\
& \leq \sum_{|\beta| \leq m-|\gamma|}\left|f^{(\beta+\gamma)}\left(\xi^{j}\right)\right| \frac{R^{|\beta|}}{\beta !} \\
& \leq C_{1} \sum_{|\beta| \leq m-|\gamma|}\left|f^{(\gamma+\beta)}\left(\xi^{j}\right)\right| \leq C_{2} \sup _{|\lambda| \leq m}\left|f^{(\lambda)}\left(\xi^{j}\right)\right| \leq C_{2}|f|_{m, A_{\xi}} .
\end{aligned}
$$

Pelo item 3 do teorema 6.0.14 temos que

$$
\sum_{j}\left|D^{\alpha} \varphi_{j}(\xi)\right| \leq C_{\alpha}\left(\operatorname{dist}(\xi, A)^{-|\alpha|}+1\right) \leq C_{\alpha}\left(\left(\frac{4}{3}\right)^{|\alpha|}+1\right)=C_{\alpha}^{\prime} .
$$

Conseqüentemente,

$$
\begin{aligned}
\left|g^{(\alpha)}(\xi)\right| & \leq \sum_{j}^{*} \sum_{\beta+\gamma=\alpha}\left(\begin{array}{c}
\alpha \\
\beta
\end{array}\right)\left|\varphi_{j}^{(\beta)}(\xi)\right|\left|f_{m}^{(\gamma)}\left(\xi, \xi^{j}\right)\right| \\
& \leq C \sum_{\beta+\gamma=\alpha} \sum_{j}^{*}\left|\varphi_{j}^{(\beta)}(\xi)\right||f|_{m, A_{\xi}} \\
& \leq C \sum_{\beta+\gamma=\alpha} C_{\beta}^{\prime}|f|_{m, A_{\xi}} \\
& =K_{\alpha}|f|_{m, A_{\xi}} .
\end{aligned}
$$

O último caso para considerarmos é $0 \leq \operatorname{dist}(\xi, A)=\left|\xi-\xi^{*}\right|<\frac{3}{4}$.

Se $\operatorname{dist}(\xi, A)=0$, então $\xi \in A$ e assim

$$
\left|D^{\alpha} g(\xi)\right|=\left|D^{\alpha} f(\xi)\right| \leq|f|_{m, A_{\xi}}, \forall|\alpha| \leq m
$$

Nesse caso segue que $|g|_{m, \xi} \leq|f|_{m, A_{\xi}}$.

Se $\operatorname{dist}(\xi, A) \neq 0$, vejamos que $f_{m}^{(\alpha)}\left(\xi, \xi^{*}\right) \leq C_{1}|f|_{m, A_{\xi}}$, onde $C_{1}$ é uma constante:

$$
\begin{aligned}
\left|f_{m}^{(\alpha)}\left(\xi, \xi^{*}\right)\right| & \leq \sum_{|\beta| \leq m-|\alpha|}\left|f^{(\alpha+\beta)}\left(\xi^{*}\right)\right| \frac{\left|\xi-\xi^{*}\right| \beta \mid}{\beta !} \\
& \leq \sum_{|\beta| \leq m-|\alpha|}\left(\frac{3}{4}\right)^{|\beta|} \frac{\left|f^{(\alpha+\beta)}\left(\xi^{*}\right)\right|}{\beta !} \\
& \leq C_{1}|f|_{m, A_{\xi}} .
\end{aligned}
$$

O que falta estimar é

$$
f_{m}^{(\gamma)}\left(\xi, \xi^{j}\right)-f_{m}^{(\gamma)}\left(\xi, \xi^{*}\right)
$$


Temos que

$$
\begin{aligned}
f_{m}^{(\gamma)}\left(\xi, \xi^{j}\right) & =\sum_{|\beta| \leq m-|\gamma|} f^{(\gamma+\beta)}\left(\xi^{j}\right) \frac{\left(\xi-\xi^{j}\right)^{\beta}}{\beta !} \\
& =\sum_{|\beta| \leq m-|\gamma|}\left(f_{m}^{(\gamma+\beta)}\left(\xi^{j}, \xi^{*}\right)+R_{m}^{(\gamma+\beta)}\left(\xi^{j}, \xi^{*}\right)\right) \frac{\left(\xi-\xi^{j}\right)^{\beta}}{\beta !} \\
& =\sum_{|\beta| \leq m-|\gamma|} f_{m}^{(\gamma+\beta)}\left(\xi^{j}, \xi^{*}\right) \frac{\left(\xi-\xi^{j}\right)^{\beta}}{\beta !}+\sum_{|\beta| \leq m-|\gamma|} R_{m}^{(\gamma+\beta)}\left(\xi^{j}, \xi^{*}\right) \frac{\left(\xi-\xi^{j}\right)^{\beta}}{\beta !}
\end{aligned}
$$

Vejamos que

$$
f_{m}^{(\gamma)}\left(\xi, \xi^{*}\right)=\sum_{|\beta| \leq m-|\gamma|} f_{m}^{(\beta+\gamma)}\left(\xi^{j}, \xi^{*}\right) \frac{\left(\xi-\xi^{j}\right)^{\beta}}{\beta !} .
$$

Como ambos são polinômios em $\xi$ de mesmo grau, basta verificar que suas derivadas em um mesmo ponto coincidem. Vejamos que elas coincidem em $\xi=\xi^{j}$.

$$
D^{\phi} f_{m}^{(\gamma)}\left(\xi, \xi^{*}\right)=\sum_{|\lambda| \leq m-|\gamma|, \phi \leq \lambda} f^{(\gamma+\lambda)}\left(\xi^{*}\right) \frac{\left(\xi-\xi^{*}\right)^{\lambda-\phi}}{(\lambda-\phi) !} .
$$

Assim, em $\xi=\xi^{j}$ vem:

$$
D^{\phi} f_{m}^{(\gamma)}\left(\xi^{j}, \xi^{*}\right)=\sum_{|\lambda| \leq m-|\gamma|, \phi \leq \lambda} f^{(\gamma+\lambda)}\left(\xi^{*}\right) \frac{\left(\xi^{j}-\xi^{*}\right)^{\lambda-\phi}}{(\lambda-\phi) !} .
$$

Definamos

$$
H(\xi)=\sum_{|\beta| \leq m-|\gamma|} f_{m}^{(\gamma+\beta)}\left(\xi^{j}, \xi^{*}\right) \frac{\left(\xi-\xi^{j}\right)^{\beta}}{\beta !}
$$

então

$$
D^{\Phi} H(\xi)=\sum_{|\beta| \leq m-|\gamma|, \Phi \leq \beta} f_{m}^{(\gamma+\beta)}\left(\xi^{j}, \xi^{*}\right) \frac{\left(\xi-\xi^{j}\right)^{\beta-\Phi}}{(\beta-\Phi) !} .
$$

$\operatorname{Em} \xi=\xi^{j}$ obtemos:

$$
D^{\Phi} H\left(\xi^{j}\right)=f_{m}^{(\Phi+\gamma)}\left(\xi^{j}, \xi^{*}\right)=\sum_{|\lambda| \leq m-|\gamma|} f^{(\gamma+\lambda)}\left(\xi^{*}\right) \frac{\left(\xi^{j}-\xi^{*}\right)^{\lambda-\Phi}}{(\lambda-\Phi) !} .
$$

Dessa forma,

$$
\left|f_{m}^{(\gamma)}\left(\xi, \xi^{j}\right)-f_{m}^{(\gamma)}\left(\xi, \xi^{*}\right)\right|=\left|\sum_{|\beta| \leq m-|\gamma|} R_{m}^{(\gamma+\beta)}\left(\xi^{j}, \xi^{*}\right) \frac{\left(\xi-\xi^{j}\right)^{\beta}}{\beta !}\right| .
$$

Veremos a seguir que

$$
\left|R_{m}^{(\gamma+\beta)}\left(\xi^{j}, \xi^{*}\right)\right| \leq|f|_{m, A_{\xi}}\left|\xi^{j}-\xi^{*}\right|^{m-|\gamma|-|\beta|} .
$$


Se $\xi^{j}=\xi^{*}$ isso é verdade: o lado direito da desigualdade é nulo e

$$
\begin{aligned}
R_{m}^{(\lambda)}\left(\xi^{*}, \xi^{*}\right) & =f^{(\lambda)}\left(\xi^{*}\right)-f_{m}^{(\lambda)}\left(\xi^{*}, \xi^{*}\right) \\
& =f^{(\lambda)}\left(\xi^{*}\right)-\sum_{|\beta| \leq m-|\lambda|} f^{(\lambda+\beta)}\left(\xi^{*}\right) \frac{\left(\xi^{*}-\xi^{*}\right)^{\beta}}{\beta !} \\
& =f^{(\lambda)}\left(\xi^{*}\right)-f^{(\lambda)}\left(\xi^{*}\right)=0 .
\end{aligned}
$$

Se $\xi^{j}, \xi^{*} \in A$, são distintos, então

$$
\left|R_{m}^{(\gamma+\beta)}\left(\xi^{j}, \xi^{*}\right)\right|=\left|R_{m}^{(\gamma+\beta)}\left(\xi^{j}, \xi^{*}\right)\right| \frac{\left|\xi^{j}-\xi^{*}\right|^{m-|\gamma|-|\beta|}}{\left|\xi^{j}-\xi^{*}\right| m-|\gamma|-|\beta|} \leq|f|_{m, A_{\xi}}\left|\xi^{j}-\xi^{*}\right|^{m-|\gamma|-|\beta|} .
$$

Assim, assumindo que $\xi \in \operatorname{supp} \varphi_{j}$,

$$
\begin{aligned}
\left|f_{m}^{(\gamma)}\left(\xi, \xi^{j}\right)-f_{m}^{(\gamma)}\left(\xi, \xi^{*}\right)\right| & \leq|f|_{m, A_{\xi}} \sum_{|\beta| \leq m-|\gamma|}\left|\xi^{j}-\xi^{*}\right|^{m-|\gamma|-|\beta|} \frac{\left|\xi-\xi^{j}\right|^{|\beta|}}{\beta !} \\
& \leq c|f|_{m, A_{\xi}} \sum_{|\beta| \leq m-|\gamma|} \operatorname{dist}(\xi, A)^{m-|\gamma|-|\beta|} \frac{\operatorname{dist}(\xi, A)^{|\beta|}}{\beta !} \\
& \leq C^{\prime}|f|_{m, A_{\xi}} \operatorname{dist}(\xi, A)^{m-|\gamma|} .
\end{aligned}
$$

Concluimos que

$$
\begin{aligned}
\left|g^{(\alpha)}(\xi)\right| & \leq\left|f_{m}^{(\alpha)}\left(\xi, \xi^{*}\right)\right|+c \sum_{\beta+\gamma=\alpha} \sum_{j}^{*}\left|\varphi_{j}^{(\beta)}(\xi)\right|\left|f_{m}^{(\gamma)}\left(\xi, \xi^{j}\right)-f_{m}^{(\gamma)}\left(\xi, \xi^{*}\right)\right| \\
& \leq C_{1}|f|_{m, A_{\xi}}+c \sum_{\beta+\gamma=\alpha} C_{\beta}\left(\operatorname{dist}(\xi, A)^{-|\beta|}+1\right) C^{\prime} \operatorname{dist}(\xi, A)^{m-|\gamma|}|f|_{m, A_{\xi}} \\
& =C_{1}|f|_{m, A_{\xi}}+C_{2} \sum_{\beta+\gamma=\alpha}\left(\operatorname{dist}(\xi, A)^{-|\beta|}+1\right) \operatorname{dist}(\xi, A)^{m-|\gamma|}|f|_{m, A_{\xi}} .
\end{aligned}
$$

Como $m-|\beta|-|\gamma|=m-|\alpha| \geq 0$, então $\operatorname{dist}(\xi, A)^{m-|\alpha|}<1$ e portanto

$$
\left|g^{(\alpha)}(\xi)\right| \leq K|f|_{m, A_{\xi}}
$$

Teorema 6.0.15 (Teorema da Extensão de Whitney) $A$ aplicação linear $f \longmapsto g$ definida como antes aplica $\mathcal{C}^{m}\left(\mathbb{R}^{n}\right)$ em $\mathcal{C}^{m}\left(\mathbb{R}^{n}\right)$ de modo que valem:

$$
D^{\alpha} g(x)=D^{\alpha} f(x), \forall \alpha \in \mathbb{N}^{n}:|\alpha| \leq m, \forall x \in A,
$$

e existe uma constante $K$, independente de $f$ e de $\xi$ de modo que

$$
|g|_{m, \xi} \leq K|f|_{m, A_{\xi}}, \forall \xi \in \mathbb{R}^{n} .
$$




\section{Capítulo 7}

\section{Prova do Teorema principal}

Nosso objetivo é provar o seguinte teorema:

Teorema 7.0.16 Se P é um polinômio em $\mathbb{R}^{n}$ que não se anula identicamente, então a aplicação multiplicação $M$ definida por

$$
\mathcal{S} \ni f \mapsto P f \in \mathcal{S}
$$

tem uma inversa contínua (definida na imagem de $M$ ).

Relembrando as observações feitas no início do capítulo sobre a Topologia de $\mathcal{S}$, a aplicação $M$ está de fato bem definida.

Vale notar que se $P$ é um polinômio constante não nulo, então a aplicação $M$ tem uma inversa contínua. Isto é imediato pois $\mathcal{S}$ é um espaço vetorial topológico.

Por conta dessa observação podemos assumir que o polinômio $P$ não é constante, e que tem grau positivo $\mu$. Seja $\mathcal{N}^{k}$ o conjunto dos pontos $\xi$ nos quais $P$ têm um zero de ordem pelo menos $k$, isto é,

$$
\mathcal{N}^{k}=\cap_{|\alpha|<k}\left\{\xi \in \mathbb{R}^{N}: P^{(\alpha)}(\xi)=0\right\} .
$$

Podemos notar que $\mathcal{N}^{k}$ é um conjunto fechado.

Como o grau de $P$ é $\mu$, existe $\alpha \in \mathbb{N}^{N}$ com $|\alpha|=\mu$ tal que $P^{(\alpha)}=C, C$ constante não nula. Dessa forma, $\mathcal{N}^{\mu+1}=\emptyset$. Além disso, é claro que $\mathcal{N}^{k+1} \subseteq \mathcal{N}^{k}$, donde obtemos que

$$
\emptyset=\mathcal{N}^{\mu+1} \subseteq \mathcal{N}^{\mu} \subseteq \ldots \subseteq \mathcal{N}^{2} \subseteq \mathcal{N}^{1} \subseteq \mathcal{N}^{0}=\mathbb{R}^{N} .
$$

A idéia da prova do teorema 7.0.16 consiste em estimar $f$ em termos de $P f$ primeiro em $\mathcal{N}^{\mu}$, depois em $\mathcal{N}^{\mu-1}$ e assim sucessivamente.

Provaremos sucessivamente, para $k$ decrescente, a seguinte afirmação:

Lema 7.0.26 Para todos $n, m, k \in \mathbb{N} \operatorname{com} k \leq \mu+1$, existem $n^{\prime}, m^{\prime} \in \mathbb{N}$ e uma constante $K$ tais que

$$
\sup _{\xi \in \mathbb{R}^{N}}(1+|\xi|)^{n}|f|_{m,\left(\mathcal{N}^{k}\right)_{\xi}} \leq K \sup _{\xi \in \mathbb{R}^{N}}(1+|\xi|)^{n^{\prime}}|P f|_{m^{\prime}, \xi}, \forall f \in \mathcal{C}^{m^{\prime}}\left(\mathbb{R}^{N}\right) .
$$


Na expressão acima pode ocorrer que $\sup _{\xi \in \mathbb{R}^{N}}(1+|\xi|)^{n}|f|_{m,\left(\mathcal{N}^{k}\right)_{\xi}}=\infty$, ou ainda que $\sup _{\xi \in \mathbb{R}^{N}}(1+$ $|\xi|)^{n^{\prime}}|P f|_{m^{\prime}, \xi}=\infty$

Iniciaremos com a prova de um resultado que será bastante útil.

Lema 7.0.27 Dados $m, n \in \mathbb{N}$, existe uma constante $C>0$ (que depende de $m$ ) tal que

$$
(1+|\xi|)^{n}|h|_{m,\left(\mathcal{N}^{k}\right)_{\xi}} \leq C \sup _{y \in \mathbb{R}^{N}}(1+|y|)^{n}|h|_{m, y}, \forall h \in \mathcal{C}^{m}\left(\mathbb{R}^{N}\right), \forall \xi \in \mathbb{R}^{N}
$$

Demonstração: Como $\left(\mathcal{N}^{k}\right)_{\xi}=B_{R}[\xi] \cap \mathcal{N}^{k} \subseteq B_{R}[\xi],(1+|\xi|)^{n}|h|_{m,\left(\mathcal{N}^{k}\right)_{\xi}} \leq(1+|\xi|)^{n}|h|_{m, B_{R}[\xi]}$. Pelo corolário 8.0.5 do Apêndice, existe uma constante $S>0$ tal que

$$
|h|_{m, B_{R}[\xi]} \leq S \sup _{|\beta| \leq m, y \in B_{R}[\xi]}\left|D^{\beta} h(y)\right|
$$

Assim,

$$
(1+|\xi|)^{n}|h|_{m, B_{R}[\xi]} \leq S \sup _{|\beta| \leq m, y \in B_{R}[\xi]}(1+|\xi|)^{n}\left|D^{\beta} h(y)\right|
$$

Dado $y \in B_{R}[\xi]$, como $1+|\xi| \leq 1+|\xi-y|+|y| \leq(R+1)(1+|y|)$,

$$
(1+|\xi|)^{n}|h|_{m, B_{R}[\xi]} \leq S(R+1)^{n} \sup _{|\beta| \leq m, y \in B_{R}[\xi]}(1+|y|)^{n}\left|D^{\beta} h(y)\right| \leq S(R+1)^{n} \sup _{y \in \mathbb{R}^{N}}(1+|y|)^{n}|h|_{m, y}
$$

uma vez que para cada $y \in B_{R}[\xi]$ e para cada $\beta \in \mathbb{N}^{N}$ tal que $|\beta| \leq m$,

$$
\begin{aligned}
(1+|y|)^{n}\left|D^{\beta} h(y)\right| & \leq(1+|y|)^{n} \sup _{|\gamma| \leq m}\left|D^{\gamma} h(y)\right| \\
& \leq \sup _{z \in \mathbb{R}^{N}}(1+|z|)^{n}|h|_{m, z}
\end{aligned}
$$

Lema 7.0.28 O lema 7.0.26 é verdadeiro se $k=\mu+1$, e $n, m \in \mathbb{N}$ são quaisquer.

Demonstração: Seja $m^{\prime} \in \mathbb{N}$ qualquer. Temos que $\mathcal{N}^{k}=\emptyset$, donde $\left(\mathcal{N}^{k}\right) \xi=\emptyset$, e portanto $|f|_{m,\left(\mathcal{N}^{k}\right) \xi}=0, \forall f \in \mathcal{C}^{m^{\prime}}\left(\mathbb{R}^{N}\right)$.

Lema 7.0.29 A afirmação do lema 7.0.26 com $k=0$ implica o teorema 7.0.16, isto é, se para todos $n, m \in \mathbb{N}$ existirem $n^{\prime}, m^{\prime} \in \mathbb{N}$ e uma constante $K$ tais que

$$
\sup _{\xi \in \mathbb{R}^{N}}(1+|\xi|)^{n}|f|_{m, \mathcal{N}^{0}}{ }_{\xi} \leq K \sup _{\xi \in \mathbb{R}^{N}}(1+|\xi|)^{n^{\prime}}|P f|_{m^{\prime}, \xi}, \forall f \in \mathcal{C}^{m^{\prime}}\left(\mathbb{R}^{N}\right),
$$

então vale o teorema 7.0.16. Além disso, o teorema 7.0.16 implica que para todos $m, n \in \mathbb{N}$ existem $m^{\prime}, n^{\prime} \in \mathbb{N}$ e uma constante positiva $K$ tais que

$$
\sup _{\xi \in \mathbb{R}^{N}}(1+|\xi|)^{n}|f|_{m, \mathcal{N}^{0} \xi} \leq K \sup _{\xi \in \mathbb{R}^{N}}(1+|\xi|)^{n^{\prime}}|P f|_{m^{\prime}, \xi}, \forall f \in \mathcal{S} .
$$


Demonstração: Sejam $\varepsilon>0$ e $L \subseteq \mathbb{N} \times \mathbb{N}$ finito. Para cada $(m, n) \in L$, sejam $K_{m, n}$ e $m^{\prime}, n^{\prime} \in \mathbb{N}$ tais que

$$
\sup _{\xi \in \mathbb{R}^{N}}(1+|\xi|)^{n}|g|_{m, \mathcal{N}^{0} \xi} \leq K_{m, n} \sup _{\xi \in \mathbb{R}^{N}}(1+|\xi|)^{n^{\prime}}|P g|_{m^{\prime}, \xi}, \forall g \in \mathcal{C}^{m^{\prime}}\left(\mathbb{R}^{N}\right) .
$$

Dados $o, p \in \mathbb{N}$, vamos denotar por $F_{o, p}$ a seguinte função (que é uma semi-norma em $\mathcal{S}$ ): $F_{o, p}(f)=$ $\sup _{\xi \in \mathbb{R}^{N}}(1+|\xi|)^{p} \sup _{|\beta| \leq o}\left|D^{\beta} f(\xi)\right|$. Como $\mathcal{N}^{0}{ }_{\xi}=\mathbb{R}^{N}{ }_{\xi}=B_{R}[\xi]$, segue que

$$
\begin{aligned}
F_{m, n}(g) & \leq \sup _{\xi \in \mathbb{R}^{N}}(1+|\xi|)^{n}|g|_{m, \mathcal{N}^{0} \xi} \\
& \leq K_{m, n} \sup _{\xi \in \mathbb{R}^{N}}(1+|\xi|)^{n^{\prime}}|P g|_{m^{\prime}, \xi} \\
& =K_{m, n} F_{m^{\prime}, n^{\prime}}(P g), \forall g \in \mathcal{C}^{m^{\prime}}\left(\mathbb{R}^{N}\right) .
\end{aligned}
$$

Sejam $K=\max \left\{K_{m, n}:(m, n) \in L\right\}, L^{\prime}=\left\{\left(m^{\prime}, n^{\prime}\right):(m, n) \in L\right\}$ e $\delta=\frac{\varepsilon}{K}$. Para todo $(m, n) \in L$ e toda $g \in \mathcal{C}^{\infty}\left(\mathbb{R}^{N}\right)$,

$$
F_{m, n}(g) \leq K F_{m^{\prime}, n^{\prime}}(P g) \leq K \sup _{\left(m^{\prime}, n^{\prime}\right) \in L^{\prime}} F_{m^{\prime}, n^{\prime}}(P g)
$$

Sejam $g \in \mathcal{S}$ e $f=P g$. Se

$$
\sup _{\left(m^{\prime}, n^{\prime}\right) \in L^{\prime}} F_{m^{\prime}, n^{\prime}}(f)=\sup _{\left(m^{\prime}, n^{\prime}\right) \in L^{\prime}} F_{m^{\prime}, n^{\prime}}(P g)<\frac{\varepsilon}{K},
$$

então $\sup _{(m, n) \in L} F_{m, n}(g)<\varepsilon$, e a aplicação inversa de $M$ é contínua.

Quanto à outra afirmação, suponhamos que o teorema 7.0.16 vale. Dados $m, n \in \mathbb{N}$, existem $\delta>0$ e $L \subseteq \mathbb{N} \times \mathbb{N}$ finito tais que se $f \in \mathcal{S}$ é tal que $\sup _{(o, p) \in L} F_{o, p}(P f)<\delta$, então $F_{m, n}(f)<1$. Sejam $m^{\prime}=\max \{o: \exists p$ tal que $(o, p) \in L\}$ e $n^{\prime}=\max \{p: \exists o$ tal que $(o, p) \in L\}$ e $K=\frac{2}{\delta}$. Note que

$$
\sup _{(o, p) \in L} F_{o, p}(g) \leq F_{m^{\prime}, n^{\prime}}(g), \forall g \in \mathcal{S} .
$$

Tome $f \in \mathcal{S}$. Se $F_{m^{\prime}, n^{\prime}}(P f)=0$, então $P f=0$, donde $f=0$ e o resultado vale obviamente. Caso contrário, sejam $g=\operatorname{Pf}$ e $h=\frac{\delta}{2} \frac{g}{F_{m^{\prime}, n^{\prime}}(g)}$. Dado $(o, p) \in L$,

$$
F_{o, p}(h)=\frac{\delta}{2} \frac{F_{o, p}(g)}{F_{m^{\prime}, n^{\prime}}(g)} \leq \frac{\delta}{2}<\delta
$$

e $\operatorname{assim} F_{m, n}\left(\frac{\delta}{2} \frac{f}{F_{m^{\prime}, n^{\prime}}(P f)}\right)<1$, isto é, $F_{m, n}(f) \leq \frac{2}{\delta} F_{m^{\prime}, n^{\prime}}(P f)$. Pelo lema 7.0.27 existe uma constante $C>0$ tal que $\sup _{\xi \in \mathbb{R}^{N}}(1+|\xi|)^{n}|f|_{m, \mathcal{N}^{0}}{ }_{\xi} \leq C F_{m, n}(f)$, e portanto

$$
\sup _{\xi \in \mathbb{R}^{N}}(1+|\xi|)^{n}|f|_{m, \mathcal{N}^{0}} \leq \frac{2 C}{\delta} F_{m^{\prime}, n^{\prime}}(P f), \forall f \in \mathcal{S} .
$$


Para provar o lema 7.0.26 vamos fixar $n, m, k \in \mathbb{N}$, onde $k$ é tal que $k+1 \leq \mu+1$. Vamos assumir que a afirmação já foi provada para $k+1$. O valor de $m^{\prime}$ será determinado depois, a partir de alguns cálculos que faremos. Por ora vamos assumir que $m^{\prime} \geq 2 k m+2 m+2 k$ (assim ele é grande o suficiente para que as contas a seguir façam sentido).

As desigualdades essenciais que provaremos são as seguintes:

- Existem constantes $n^{\prime}, K>0$ tais que se $f \in \mathcal{C}^{l}\left(\mathbb{R}^{N}\right.$ ) se anula de ordem $l$ em $\mathcal{N}^{k+1}$ (para $l$ suficientemente grande), então

$$
\left|f^{(\alpha)}(\xi)\right| \leq K(1+|\xi|)^{n^{\prime}}|P f|_{l, B_{1}[\xi]}, \forall \xi \in \mathcal{N}^{k}, \forall \alpha \in \mathbb{N}^{N}:|\alpha| \leq m
$$

- Existem constantes $K^{\prime \prime}, n^{\prime \prime}>0$ tais que se $f \in \mathcal{C}^{s}\left(\mathbb{R}^{N}\right)$ se anula de ordem $s$ em $\mathcal{N}^{k+1}$ (onde $s$ é suficientemente grande) e $\xi, \eta \in \mathcal{N}^{k}$ são tais que

$$
\operatorname{dist}\left(\xi, \mathcal{N}^{k+1}\right)+\operatorname{dist}\left(\eta, \mathcal{N}^{k+1}\right) \leq 2|\xi-\eta|,
$$

então se $S(\xi, \eta)=B_{1}[\xi] \cup B_{1}[\eta]$, teremos que

$$
\left|R_{m}^{(\alpha)}(\xi ; \eta)\right| \leq K^{\prime \prime}(1+|\xi|+|\eta|)^{n^{\prime \prime}}|\xi-\eta|^{m}|P f|_{s, S(\xi, \eta)}, \forall \alpha \in \mathbb{N}^{N}:|\alpha| \leq m
$$

- Existem constantes $K^{\prime \prime \prime}, n^{\prime \prime \prime}>0$ tais que se $f \in \mathcal{C}^{p}\left(\mathbb{R}^{N}\right)$ se anula de ordem $p$ em $\mathcal{N}^{k+1}$, (onde $p$ é suficientemente grande) e $\xi, \eta \in \mathcal{N}^{k}$ são tais que

$$
2|\xi-\eta|<\operatorname{dist}\left(\xi, \mathcal{N}^{k+1}\right)+\operatorname{dist}\left(\eta, \mathcal{N}^{k+1}\right),
$$

então, se $S_{1}(\xi, \eta)$ é o conjunto dos pontos cuja distância ao segmento que une $\xi$ e $\eta$ é no máximo 1 ,

$$
\left|R_{m}^{(\alpha)}(\xi ; \eta)\right| \leq K^{\prime \prime \prime}|\xi-\eta|^{m-|\alpha|}(1+|\xi|+|\eta|)^{n^{\prime \prime \prime}}|F|_{p, S_{1}(\xi, \eta)}, \forall \alpha \in \mathbb{N}^{N}:|\alpha| \leq m .
$$

Dada uma função $f \in \mathcal{C}^{m^{\prime}}\left(\mathbb{R}^{N}\right)$, definamos

$$
F(\xi)=P(\xi) f(\xi) .
$$

Aplicando o operador diferencial $D^{\beta}$, com $|\beta|=k$, temos:

$$
F^{(\beta)}(\xi)=P^{(\beta)}(\xi) f(\xi), \forall \xi \in \mathcal{N}^{k},
$$

pois se $\xi \in \mathcal{N}^{k}, P^{(\gamma)}(\xi)=0, \forall \gamma \in \mathbb{N}^{N}$ tal que $|\gamma|<k$.

Com o intuito de obter uma fórmula semelhante para $f^{(\alpha)}(\xi)$, onde $|\alpha| \leq m$, escrevemos

$$
f(\xi)=\frac{F(\xi)}{P(\xi)}, \forall \xi \in \mathbb{R}^{N}: P(\xi) \neq 0 .
$$

Seja $\alpha \in \mathbb{N}^{N}$ tal que $|\alpha| \leq m$. Quando $P(\xi) \neq 0$ aplicamos o operador diferencial $D^{\alpha}$ em ambos os lados. Teremos:

$$
f^{(\alpha)}(\xi)=\sum_{\gamma \leq \alpha}\left(\begin{array}{l}
\alpha \\
\gamma
\end{array}\right) D^{\alpha-\gamma} F(\xi) D^{\gamma} \frac{1}{P}(\xi)
$$


Para cada $\gamma \leq \alpha, D^{\gamma} \frac{1}{P}(\xi)$ pode ser escrito como uma soma de termos cujos denominadores são da forma $P(\xi)^{|\lambda|}$, onde $|\lambda| \leq|\gamma|+1 \leq|\alpha|+1$. Multiplicando então ambos os lados por $P(\xi)^{|\alpha|+1}$ podemos escrever:

$$
P(\xi)^{|\alpha|+1} f^{(\alpha)}(\xi)=L_{|\alpha|}(\xi, F), \forall \xi \in \mathbb{R}^{N},
$$

onde $L_{s}(\xi,$.$) denotará um operador diferencial de ordem no máximo s$ cujos coeficientes são polinômios em $\xi$, isto é,

$$
L_{s}(\xi, .)=\sum_{|\gamma| \leq s} Q_{\gamma}(\xi) D^{\gamma}
$$

sendo cada $Q_{\gamma}$ um polinômio em $\xi$.

Note que pudemos escrever que (7.0.2) vale para todo $\xi \in \mathbb{R}^{N}$ pois se $P(\xi)=0, F(\xi)=0$.

Aplicando agora o operador diferencial $D^{\beta}$, com $|\beta|=(|\alpha|+1) k$, teremos:

$$
D^{\beta}\left\{P(\xi)^{|\alpha|+1} f^{(\alpha)}(\xi)\right\}=L_{k|\alpha|+k+|\alpha|}(\xi, F)=D^{\beta}\left\{P(\xi)^{|\alpha|+1}\right\} f^{(\alpha)}(\xi), \forall \xi \in \mathcal{N}^{k},
$$

uma vez que $P^{(\gamma)}(\xi)=0$ se $|\gamma|<k$ quando $\xi \in \mathcal{N}^{k}$.

Como vimos,

$$
R_{m}^{(\alpha)}(\xi ; \eta)=f^{(\alpha)}(\xi)-\sum_{|\beta| \leq m-|\alpha|} f^{(\alpha+\beta)}(\eta) \frac{(\xi-\eta)^{\beta}}{\beta !} .
$$

Multiplicando a linha de cima por $P(\xi)^{|\alpha|+1} P(\eta)^{m+1}$ teremos:

$$
\begin{gathered}
P(\eta)^{m+1} L_{|\alpha|}(\xi, F)-P(\xi)^{|\alpha|+1} \sum_{|\beta| \leq m-|\alpha|} P(\eta)^{|\alpha+\beta|+1} P(\eta)^{-|\alpha+\beta|+m} f^{(\alpha+\beta)}(\eta) \frac{(\xi-\eta)^{\beta}}{\beta !} \\
=P(\eta)^{m+1} L_{|\alpha|}(\xi, F)-P(\xi)^{|\alpha|+1} \sum_{|\beta| \leq m-|\alpha|} P(\eta)^{m-|\alpha|-|\beta|} L_{|\alpha|+|\beta|}(\eta, F) \frac{(\xi-\eta)^{\beta}}{\beta !} \\
=P(\xi)^{|\alpha|+1} P(\eta)^{m+1} R_{m}^{(\alpha)}(\xi ; \eta)=L_{|\alpha|, m}(\xi, \eta, F), \forall \xi, \eta \in \mathbb{R}^{N},
\end{gathered}
$$

se definirmos que $L_{s, t}(\xi, \eta, F)$ é uma combinação linear das derivadas de $F$ em $\xi$ e em $\eta$ de ordens respectivamente menores ou iguais a $s$ e a $t$ com coeficientes que são polinômios em $\xi$ e em $\eta$, isto é,

$$
L_{s, t}(\xi, \eta, F)=\sum_{|\gamma| \leq s} Q_{\gamma}(\xi, \eta) D^{\gamma} F(\xi)+\sum_{|\lambda| \leq t} R_{\lambda}(\xi, \eta) D^{\lambda} F(\eta),
$$

onde $Q_{\gamma}(\xi, \eta)$ e $R_{\lambda}(\xi, \eta)$ são polinômios em $\xi$ e em $\eta$.

Vejamos que as derivadas de

$$
L_{|\alpha|, m}(\xi, \eta, F)=P(\xi)^{|\alpha|+1} P(\eta)^{m+1} R_{m}^{(\alpha)}(\xi ; \eta)
$$

com respeito a $\xi$ e a $\eta$ de ordens totais menores que $k(|\alpha|+1)+k(m+1)+m-|\alpha|$ se anulam em $(\xi, \xi)$, para todo $\xi \in \mathcal{N}^{k}$.

Para obter um termo não nulo na expressão dada pela fórmula de Leibniz devemos diferenciar o fator $P(\xi)^{|\alpha|+1}$ com relação a $\xi$ de modo que o comprimento total seja pelo menos $k(|\alpha|+1)$; devemos diferenciar o fator $P(\eta)^{m+1}$ com relação a $\eta$ de modo que o comprimento seja ao menos 
$k(m+1)$. Quanto ao último fator, segue do lema 8.0.39 do Apêndice que as derivadas de $R_{m}^{(\alpha)}(\xi ; \eta)$ com respeito a $\xi$ e a $\eta$ de ordens menores ou iguais a $m-|\alpha|$ se anulam em $(\xi, \xi)$, se $\xi \in \mathcal{N}^{k}$.

Aplicando em (7.0.3) os operadores diferenciais $D^{\beta}$ com relação a $\xi$ e $D^{\gamma}$ com relação a $\eta$ em $L_{|\alpha|, m}(\xi, \eta, F), \operatorname{com}|\beta|=(|\alpha|+1) k,|\gamma|=(m+1) k$, vamos definir

$$
L_{k|\alpha|+k+|\alpha|, k m+k+m}(\xi, \eta, F)=D_{\xi}^{\beta} D_{\eta}^{\gamma} L_{|\alpha|, m}(\xi, \eta, F) .
$$

Assim

$$
L_{k|\alpha|+k+|\alpha|, k m+k+m}(\xi, \eta, F)=\left\{D^{\beta}\left(P(\xi)^{|\alpha|+1}\right)\right\}\left\{D^{\gamma}\left(P(\eta)^{m+1}\right)\right\} R_{m}^{(\alpha)}(\xi ; \eta), \forall \xi, \eta \in \mathcal{N}^{k} .
$$

Voltando ao lema 7.0.26, provaremos que ele é verdadeiro desde que acrescentemos a hipótese adicional de que $f$ se anula de ordem $m^{\prime}$ em $\mathcal{N}^{k+1}$. Note que se $\mathcal{N}^{k+1}=\emptyset$ isso não é nenhuma restrição. A prova de tal afirmação seguirá do teorema 5.0.13 e de algumas fórmulas que provaremos. O Teorema da Extensão de Whitney e a hipótese de indução de que (7.0.1) é válida com $k+1$ no lugar de $k$ nos permitirão concluir a prova.

Inicialmente vamos supor que $\mathcal{N}^{k+1} \neq \emptyset$. Para $j \in \mathbb{N}^{*}$, seja

$$
Q_{j}(\xi)=\sum_{|\beta| \leq j k}\left|D^{\beta}\left(P(\xi)^{j}\right)\right|^{2} .
$$

Pelo lema 8.0.40 do Apêndice, $\left\{x \in \mathbb{R}^{N}: Q_{j}(x)=0\right\}=\mathcal{N}^{k+1}$. Pelo teorema 5.0.13 existem constantes positivas $c_{j}, a_{j}, \mu_{j}^{\prime \prime}$ tais que

$$
\left|Q_{j}(\xi)\right| \geq c_{j}\left(1+|\xi|^{2}\right)^{-\mu_{j}^{\prime \prime}} \operatorname{dist}\left(\xi, \mathcal{N}^{k+1}\right)^{a_{j}}, \forall \xi \in \mathbb{R}^{N},
$$

isto é, se $\mu_{j}^{\prime}=\frac{a_{j}}{2}$,

$$
\begin{aligned}
\sum_{|\beta| \leq j k}\left|D^{\beta}\left(P(\xi)^{j}\right)\right|^{2} & \geq c_{j}\left(1+|\xi|^{2}\right)^{-\mu_{j}^{\prime \prime}} \operatorname{dist}\left(\xi, \mathcal{N}^{k+1}\right)^{a_{j}} \\
& =c_{j}\left(1+|\xi|^{2}\right)^{-\mu_{j}^{\prime \prime}} \operatorname{dist}\left(\xi, \mathcal{N}^{k+1}\right)^{2 \mu_{j}^{\prime}}, \forall \xi \in \mathbb{R}^{N}
\end{aligned}
$$

Assim sendo,

$$
\sum_{|\beta|=j k}\left|D^{\beta}\left(P(\xi)^{j}\right)\right|^{2} \geq c_{j} \operatorname{dist}\left(\xi, \mathcal{N}^{k+1}\right)^{2 \mu_{j}^{\prime}}\left(1+|\xi|^{2}\right)^{-\mu_{j}^{\prime \prime}}, \forall \xi \in \mathcal{N}^{k}
$$

pois as derivadas de $P^{j}$ de ordens menores que $j k$ se anulam em $\xi \in \mathcal{N}^{k}$. Pelo lema 8.0.41 do Apêndice, existem constantes $c, \mu^{\prime}, \mu^{\prime \prime}>0$ tais que

$$
\sum_{|\beta|=j k}\left|D^{\beta}\left(P(\xi)^{j}\right)\right|^{2} \geq c \operatorname{dist}\left(\xi, \mathcal{N}^{k+1}\right)^{2 \mu^{\prime}}\left(1+|\xi|^{2}\right)^{-\mu^{\prime \prime}}, \forall \xi \in \mathcal{N}^{k}, \forall j \leq(m+1) .
$$

Seja $l \in \mathbb{N}$ tal que $\mu^{\prime}+k m+k+m \leq l$.

Lema 7.0.30 Existem constantes $n^{\prime}, K>0$ tais que se $f \in \mathcal{C}^{l}\left(\mathbb{R}^{N}\right)$ se anula de ordem $l$ em $\mathcal{N}^{k+1}$,

$$
\left|f^{(\alpha)}(\xi)\right| \leq K(1+|\xi|)^{n^{\prime}}|F|_{l, B_{1}[\xi]}, \forall \xi \in \mathcal{N}^{k}, \forall \alpha \in \mathbb{N}^{N}:|\alpha| \leq m
$$


Demonstração: Comecemos fixando $\alpha \in \mathbb{N}^{N}$ tal que $|\alpha| \leq m$. Pelo lema 8.0.42 do Apêndice, existem constantes $K_{1}>0, n_{1} \in \mathbb{N}$ tais que se $f \in \mathcal{C}^{l}\left(\mathbb{R}^{N}\right)$ se anula de ordem $l$ em $\mathcal{N}^{k+1}$ e $\beta \in \mathbb{N}^{N}$ é tal que $|\beta|=(|\alpha|+1) k$, então

$$
\left|L_{k|\alpha|+k+|\alpha|}^{\beta}(\xi, F)\right| \leq K_{1}(1+|\xi|)^{n_{1}} \operatorname{dist}\left(\xi, \mathcal{N}^{k+1}\right)^{l-k|\alpha|-k-|\alpha|}|F|_{l, B_{1}[\xi]}, \forall \xi \in \mathbb{R}^{N},
$$

onde escrevemos $L_{k|\alpha|+k+|\alpha|}^{\beta}(\xi, F)$ para frisar que $L_{k|\alpha|+k+|\alpha|}(\xi, F)$ depende de $\beta$.

Se $\beta \in \mathbb{N}^{N}$ é tal que $|\beta|=(|\alpha|+1) k$ e $\xi \in \mathcal{N}^{k}$,

$$
\left|\left\{D^{\beta}\left(P(\xi)^{|\alpha|+1}\right)\right\} f^{(\alpha)}(\xi)\right|^{2}=\left|L_{k|\alpha|+k+|\alpha|}^{\beta}(\xi, F)\right|^{2} .
$$

Somando a igualdade de cima para todos os $\beta \in \mathbb{N}^{N}$ tais que $|\beta|=(|\alpha|+1) k$ e tirando a raíz quadrada teremos, como conseqüência de (7.0.4), que existe uma constante $K_{2}$ tal que se $f \in \mathcal{C}^{l}\left(\mathbb{R}^{N}\right)$ se anula de ordem $l$ em $\mathcal{N}^{k+1}$, então para todo $\xi \in \mathcal{N}^{k}$,

$$
\left\{\sum_{|\beta|=(|\alpha|+1) k}\left|D^{\beta}\left(P(\xi)^{|\alpha|+1}\right)\right|^{2}\right\}^{\frac{1}{2}}\left|f^{(\alpha)}(\xi)\right| \leq K_{2}(1+|\xi|)^{n_{1}} \operatorname{dist}\left(\xi, \mathcal{N}^{k+1}\right)^{l-k|\alpha|-k-|\alpha|}|F|_{l, B_{1}[\xi]} .
$$

Como

$$
\sum_{|\beta|=j k}\left|D^{\beta}\left(P(\xi)^{j}\right)\right|^{2} \geq c \operatorname{dist}\left(\xi, \mathcal{N}^{k+1}\right)^{2 \mu^{\prime}}\left(1+|\xi|^{2}\right)^{-\mu^{\prime \prime}}, \forall \xi \in \mathcal{N}^{k}, \forall j \leq(m+1),
$$

fazendo $j=|\alpha|+1$ teremos que para todo $\alpha \in \mathbb{N}^{N}$ tal que $|\alpha| \leq m$

$$
\sqrt{c} \cdot \operatorname{dist}\left(\xi, \mathcal{N}^{k+1}\right)^{\mu^{\prime}}\left(1+|\xi|^{2}\right)^{\frac{-\mu^{\prime \prime}}{2}}\left|f^{(\alpha)}(\xi)\right| \leq\left\{\sum_{|\beta|=(|\alpha|+1) k}\left|D^{\beta}\left(P(\xi)^{|\alpha|+1}\right)\right|^{2}\right\}^{\frac{1}{2}}\left|f^{(\alpha)}(\xi)\right|, \forall \xi \in \mathcal{N}^{k} .
$$

Sejam $K_{3}=K_{2} c^{\frac{-1}{2}}$ e $n_{2}=n_{1}+2 \mu^{\prime \prime}$

$$
\begin{aligned}
\left|f^{(\alpha)}(\xi)\right| & \leq K_{3}(1+|\xi|)^{n_{1}}\left(1+|\xi|^{2}\right)^{\frac{\mu^{\prime \prime}}{2}} \operatorname{dist}\left(\xi, \mathcal{N}^{k+1}\right)^{l-k|\alpha|-k-|\alpha|-\mu^{\prime}}|F|_{l, B_{1}[\xi]} \\
& \leq K_{3}(1+|\xi|)^{n_{1}}\left(1+|\xi|^{2}\right)^{\mu^{\prime \prime}} \operatorname{dist}\left(\xi, \mathcal{N}^{k+1}\right)^{l-k|\alpha|-k-|\alpha|-\mu^{\prime}}|F|_{l, B_{1}[\xi]} \\
& \leq K_{3}(1+|\xi|)^{n_{1}}(1+|\xi|)^{2 \mu^{\prime \prime}} \operatorname{dist}\left(\xi, \mathcal{N}^{k+1}\right)^{l-k|\alpha|-k-|\alpha|-\mu^{\prime}}|F|_{l, B_{1}[\xi]} \\
& \leq K_{3}(1+|\xi|)^{n_{2}} \operatorname{dist}\left(\xi, \mathcal{N}^{k+1}\right)^{l-k|\alpha|-k-|\alpha|-\mu^{\prime}}|F|_{l, B_{1}[\xi]}, \forall \xi \in \mathcal{N}^{k} .
\end{aligned}
$$

Assim, se assumirmos que $\operatorname{dist}\left(\xi, \mathcal{N}^{k+1}\right) \leq 1$, como $0 \leq l-k|\alpha|-k-|\alpha|-\mu^{\prime}$ segue que

$$
\left|f^{(\alpha)}(\xi)\right| \leq K_{3}(1+|\xi|)^{n_{2}}|F|_{l, B_{1}[\xi]}
$$

Se $\operatorname{dist}\left(\xi, \mathcal{N}^{k+1}\right) \geq 1$, é simples de se ver, analisando a prova do lema 8.0.42 do Apêndice, que existem constantes $H>0, p \in \mathbb{N}$ independentes de $f$ tais que 
$\left|L_{k|\alpha|+k+|\alpha|}^{\beta}(\xi, F)\right| \leq H(1+|\xi|)^{p}|F|_{l, B_{1}[\xi]}, \forall \beta \in \mathbb{N}^{N}:|\beta|=(|\alpha|+1) k, \forall \xi \in \mathbb{R}^{N}: \operatorname{dist}\left(\xi, \mathcal{N}^{k+1}\right) \geq 1$.

Conseqüentemente, existe $L>0$ tal que

$$
\left\{\sum_{|\beta|=(|\alpha|+1) k}\left|D^{\beta}\left(P(\xi)^{|\alpha|+1}\right)\right|^{2}\right\}^{\frac{1}{2}}\left|f^{(\alpha)}(\xi)\right| \leq L(1+|\xi|)^{p}|F|_{l, B_{1}[\xi]}, \forall \xi \in \mathcal{N}^{k}: \operatorname{dist}\left(\xi, \mathcal{N}^{k+1}\right) \geq 1
$$

donde

$$
\begin{aligned}
\left|f^{(\alpha)}(\xi)\right| & \leq \frac{L}{\sqrt{c}} \operatorname{dist}\left(\xi, \mathcal{N}^{k+1}\right)^{-\mu^{\prime}}(1+|\xi|)^{\frac{\mu^{\prime \prime}}{2}+p}|F|_{l, B_{1}[\xi]} \\
& \leq \frac{L}{\sqrt{c}}(1+|\xi|)^{\mu^{\prime \prime}+p}|F|_{l, B_{1}[\xi]},
\end{aligned}
$$

uma vez que $\operatorname{dist}\left(\xi, \mathcal{N}^{k+1}\right)^{-\mu^{\prime}} \leq 1$.

Sejam $K_{\alpha}=\max \left\{\frac{L}{\sqrt{c}}, K_{3}\right\}, n_{\alpha}^{\prime}=\max \left\{p+\mu^{\prime \prime}, n_{2}\right\}, K=\max \left\{K_{\alpha}: \alpha \in \mathbb{N}^{N}\right.$ e $\left.|\alpha| \leq m\right\}$ e $n^{\prime}=\max \left\{n_{\alpha}^{\prime}: \alpha \in \mathbb{N}^{N}\right.$ e $\left.|\alpha| \leq m\right\}$. Com essas constantes o lema é verdadeiro.

Agora desejamos obter estimativas semelhantes à do último lema para

$$
\frac{R_{m}^{(\alpha)}(\xi ; \eta)}{|\xi-\eta|^{m-|\alpha|}}: \xi, \eta \in \mathcal{N}^{k}: \xi \neq \eta
$$

Inicialmente, vamos assumir que $\xi, \eta \in \mathcal{N}^{k}$ são tais que $\xi \neq \eta$ e, além disso,

$$
\operatorname{dist}\left(\xi, \mathcal{N}^{k+1}\right)+\operatorname{dist}\left(\eta, \mathcal{N}^{k+1}\right) \leq 2|\xi-\eta| \text {. }
$$

Lema 7.0.31 Seja $r \in \mathbb{N}$ tal que $m+\mu^{\prime}+k m+k+m \leq r$. Existem constantes $K^{\prime}, n_{1}^{\prime}$ positivas tais que se $f \in \mathcal{C}^{r}\left(\mathbb{R}^{N}\right)$ se anula de ordem $r$ em $\mathcal{N}^{k+1}$,

$$
\left|f^{(\alpha)}(z)\right| \leq K^{\prime}(1+|z|)^{n_{1}^{\prime}} \operatorname{dist}\left(z, \mathcal{N}^{k+1}\right)^{m}|P f|_{r, B_{1}[z]}, \forall z \in \mathcal{N}^{k}, \forall \alpha \in \mathbb{N}^{N}:|\alpha| \leq m .
$$

Demonstração: Fixe $\alpha \in \mathbb{N}^{N}$ tal que $|\alpha| \leq m$. Na prova do lema anterior vimos que existem constantes $n^{\prime \prime}, K^{\prime \prime}>0$ tais que se $f \in \mathcal{C}^{r}\left(\mathbb{R}^{N}\right)$ se anula de ordem $r$ em $\mathcal{N}^{k+1}$, então

$$
\left|f^{(\alpha)}(z)\right| \leq K^{\prime \prime}(1+|z|)^{n^{\prime \prime}} \operatorname{dist}\left(z, \mathcal{N}^{k+1}\right)^{r-\mu^{\prime}-k|\alpha|-k-|\alpha|}|P f|_{r, B_{1}[\xi]}, \forall z \in \mathcal{N}^{k}
$$

Se $\operatorname{dist}\left(z, \mathcal{N}^{k+1}\right) \leq 1$, como $m+\mu^{\prime}+k m+k+m \leq r$ então $\operatorname{dist}\left(z, \mathcal{N}^{k+1}\right)^{r-\mu^{\prime}-k|\alpha|-k-|\alpha|} \leq$ $\operatorname{dist}\left(z, \mathcal{N}^{k+1}\right)^{m}$. Conseqüentemente,

$$
\left|f^{(\alpha)}(z)\right| \leq K^{\prime \prime}(1+|z|)^{n^{\prime \prime}} \operatorname{dist}\left(z, \mathcal{N}^{k+1}\right)^{m}|P f|_{r, B_{1}[\xi]} .
$$

Por outro lado, se $\operatorname{dist}\left(z, \mathcal{N}^{k+1}\right) \geq 1$, vimos também na prova do lema anterior que existem constantes positivas $L$ e $n^{*}$ tais que se $f \in \mathcal{C}^{r}\left(\mathbb{R}^{N}\right)$ se anula de ordem $r$ em $\mathcal{N}^{k+1}$, então

$$
\left|f^{(\alpha)}(z)\right| \leq L(1+|z|)^{n^{*}}|F|_{r, B_{1}[z]} \leq L(1+|z|)^{n^{*}}|F|_{r, B_{1}[z]} \operatorname{dist}\left(z, \mathcal{N}^{k+1}\right)^{m} .
$$


Fazendo $K_{\alpha}=\max \left\{K^{\prime \prime}, L\right\}, n_{\alpha}=\max \left\{n^{\prime \prime}, n^{*}\right\}$ teremos que se $f \in \mathcal{C}^{r}\left(\mathbb{R}^{N}\right)$ se anula de ordem $r$ em $\mathcal{N}^{k+1}$,

$$
\left|f^{(\alpha)}(z)\right| \leq K_{\alpha}(1+|z|)^{n_{\alpha}} \operatorname{dist}\left(z, \mathcal{N}^{k+1}\right)^{m}|P f|_{r, B_{1}[\xi]}, \forall z \in \mathcal{N}^{k}, \forall \alpha \in \mathbb{N}^{N}:|\alpha| \leq m .
$$

Definindo $K^{\prime}$ como o máximo dos $K_{\alpha}$ e $n^{\prime}$ como o máximo dos $n_{\alpha}$ quando $|\alpha| \leq m$ obtemos o resultado.

Lema 7.0.32 Seja $s \in \mathbb{N}$ tal que $m+\mu^{\prime}+k m+k+m \leq s$. Então existem constantes $K^{\prime \prime}, n^{\prime \prime}>0$ tais que se $f \in \mathcal{C}^{s}\left(\mathbb{R}^{N}\right)$ se anula de ordem s em $\mathcal{N}^{k+1}$ e $\xi, \eta \in \mathcal{N}^{k}$ são tais que

$$
\operatorname{dist}\left(\xi, \mathcal{N}^{k+1}\right)+\operatorname{dist}\left(\eta, \mathcal{N}^{k+1}\right) \leq 2|\xi-\eta|,
$$

então se $S(\xi, \eta)=B_{1}[\xi] \cup B_{1}[\eta]$, teremos que

$$
\left|R_{m}^{(\alpha)}(\xi ; \eta)\right| \leq K^{\prime \prime}(1+|\xi|+|\eta|)^{n^{\prime \prime}}|\xi-\eta|^{m}|P f|_{s, S(\xi, \eta)}, \forall \alpha \in \mathbb{N}^{N}:|\alpha| \leq m
$$

Demonstração: Pelo lema 7.0.31 existem constantes positivas $K^{\prime}, n_{1}^{\prime}$ tais que se $f \in \mathcal{C}^{s}\left(\mathbb{R}^{N}\right)$ se anula de ordem $s$ em $\mathcal{N}^{k+1}$ e $\xi, \eta \in \mathcal{N}^{k}$ são tais que $\operatorname{dist}\left(\xi, \mathcal{N}^{k+1}\right) \leq 2|\xi-\eta|$ e $\operatorname{dist}\left(\eta, \mathcal{N}^{k+1}\right) \leq$ $2|\xi-\eta|$

$$
\begin{aligned}
& \left|f^{(\alpha)}(\xi)\right| \leq K^{\prime}(1+|\xi|)^{n_{1}^{\prime}}(2|\xi-\eta|)^{m}|P f|_{s, B_{1}[\xi]}, \forall \alpha \in \mathbb{N}^{N}:|\alpha| \leq m, \\
& \left|f^{(\alpha)}(\eta)\right| \leq K^{\prime}(1+|\eta|)^{n_{1}^{\prime}}(2|\xi-\eta|)^{m}|P f|_{s, B_{1}[\eta]}, \forall \alpha \in \mathbb{N}^{N}:|\alpha| \leq m .
\end{aligned}
$$

Temos que $R_{m}^{(\alpha)}(\xi ; \eta)$ é uma combinação linear de derivadas de $f$ em $\xi$ e em $\eta$ de ordens menores ou iguais a $m$ multiplicadas por polinômios em $\xi$ e em $\eta$. Digamos que

$$
R_{m}^{(\alpha)}(\xi ; \eta)=\sum_{|\beta| \leq m} Q_{\beta}(\xi, \eta) D^{\beta} f(\xi)+\sum_{|\gamma| \leq m} T_{\gamma}(\xi, \eta) D^{\gamma} f(\eta)
$$

onde $Q_{\beta}(\xi, \eta), T_{\gamma}(\xi, \eta)$ são polinômios em $\xi$ e $\eta$.

Então existem constantes $C_{\beta}, O_{\gamma}, p_{\beta}, q_{\gamma}$ tais que

$$
\begin{aligned}
\left|R_{m}^{(\alpha)}(\xi ; \eta)\right| \leq & \sum_{|\beta| \leq m} C_{\beta}(1+|\xi|+|\eta|)^{p_{\beta}}\left|D^{\beta} f(\xi)\right|+\sum_{|\gamma| \leq m} O_{\gamma}(1+|\xi|+|\eta|)^{q_{\gamma}}\left|D^{\gamma} f(\eta)\right| \\
& \leq 2^{m} K^{\prime} \sum_{|\beta| \leq m} C_{\beta}(1+|\xi|+|\eta|)^{p_{\beta}}(1+|\xi|)^{n_{1}^{\prime}}|\xi-\eta|^{m}|P f|_{s, B_{1}[\xi]}+ \\
& +2^{m} K^{\prime} \sum_{|\gamma| \leq m} O_{\gamma}(1+|\xi|+|\eta|)^{q_{\gamma}}(1+|\eta|)^{n_{1}^{\prime}}|\xi-\eta|^{m}|P f|_{s, B_{1}[\eta]} \\
& \leq C^{\prime}(1+|\xi|+|\eta|)^{p}|\xi-\eta|^{m}|P f|_{s, S(\xi, \eta)},
\end{aligned}
$$

onde $p=n_{1}^{\prime}+\max \left\{p_{\beta}, q_{\gamma}:|\beta| \leq m,|\gamma| \leq m\right\}$.

Basta então definir $K^{\prime \prime}$ como o máximo das constantes $C^{\prime}$ quando $|\alpha| \leq m$ e $n^{\prime \prime}$ o máximo dos $p$ quando $|\alpha| \leq m$. 
Lema 7.0.33 Seja $p \in \mathbb{N}$ tal que $2 m+2 k+2 k m+2 \mu^{\prime} \leq p$. Existem constantes $K^{\prime \prime \prime}, n^{\prime \prime \prime}>0$ tais que se $f \in \mathcal{C}^{p}\left(\mathbb{R}^{N}\right)$ se anula de ordem $p$ em $\mathcal{N}^{k+1}$, e $\xi, \eta \in \mathcal{N}^{k}$ são tais que

$$
2|\xi-\eta|<\operatorname{dist}\left(\xi, \mathcal{N}^{k+1}\right)+\operatorname{dist}\left(\eta, \mathcal{N}^{k+1}\right),
$$

então, se $S_{1}(\xi, \eta)$ é o conjunto dos pontos cuja distância ao segmento que une $\xi$ e $\eta$ é no máximo 1 ,

$$
\left|R_{m}^{(\alpha)}(\xi ; \eta)\right| \leq K^{\prime \prime \prime}|\xi-\eta|^{m-|\alpha|}(1+|\xi|+|\eta|)^{n^{\prime \prime \prime}}|F|_{p, S_{1}(\xi, \eta)}, \forall \alpha \in \mathbb{N}^{N}:|\alpha| \leq m .
$$

\section{Demonstração: Como}

$$
\left|\operatorname{dist}\left(\xi, \mathcal{N}^{k+1}\right)-\operatorname{dist}\left(\eta, \mathcal{N}^{k+1}\right)\right| \leq|\xi-\eta|
$$

temos que

$$
-2|\xi-\eta| \leq 2 \operatorname{dist}\left(\xi, \mathcal{N}^{k+1}\right)-2 \operatorname{dist}\left(\eta, \mathcal{N}^{k+1}\right) \leq 2|\xi-\eta| .
$$

Por (7.0.5) segue que

$$
\frac{\operatorname{dist}\left(\eta, \mathcal{N}^{k+1}\right)}{3} \leq \operatorname{dist}\left(\xi, \mathcal{N}^{k+1}\right) \leq 3 \operatorname{dist}\left(\eta, \mathcal{N}^{k+1}\right) .
$$

Seja $\zeta$ um elemento do segmento que une $\xi$ e $\eta$. Digamos que $\zeta=(1-t) \xi+t \eta$, onde $0 \leq t \leq 1$. Notemos que $|\zeta-\xi|=|(1-t) \xi+t \eta-\xi|=t|\eta-\xi| \leq|\eta-\xi|$.

Pelas equações (7.0.5) e (7.0.6),

$$
\begin{aligned}
2|\xi-\eta| & <\operatorname{dist}\left(\xi, \mathcal{N}^{k+1}\right)+\operatorname{dist}\left(\eta, \mathcal{N}^{k+1}\right) \\
& \leq \operatorname{dist}\left(\xi, \mathcal{N}^{k+1}\right)+3 \operatorname{dist}\left(\xi, \mathcal{N}^{k+1}\right) \\
& =4 \operatorname{dist}\left(\xi, \mathcal{N}^{k+1}\right) .
\end{aligned}
$$

Conseqüentemente,

$$
\begin{aligned}
\operatorname{dist}\left(\zeta, \mathcal{N}^{k+1}\right) & \leq|\zeta-\xi|+\operatorname{dist}\left(\xi, \mathcal{N}^{k+1}\right) \\
& \leq|\eta-\xi|+\operatorname{dist}\left(\xi, \mathcal{N}^{k+1}\right) \\
& \leq 2 \operatorname{dist}\left(\xi, \mathcal{N}^{k+1}\right)+\operatorname{dist}\left(\xi, \mathcal{N}^{k+1}\right) \\
& \leq 3 \operatorname{dist}\left(\xi, \mathcal{N}^{k+1}\right)
\end{aligned}
$$

Utilizando esse argumento obtemos:

$$
\operatorname{dist}\left(\zeta, \mathcal{N}^{k+1}\right) \leq 3 \operatorname{dist}\left(\eta, \mathcal{N}^{k+1}\right), \operatorname{dist}\left(\zeta, \mathcal{N}^{k+1}\right) \leq 3 \operatorname{dist}\left(\xi, \mathcal{N}^{k+1}\right) .
$$

Para $\eta \in \mathcal{N}^{k}$ fixado, seja

$$
H(\zeta)=L_{k|\alpha|+k+|\alpha|, k m+k+m}(\zeta, \eta, F) .
$$

Vimos que $H$ se anula de ordem $m-|\alpha|$ para $\zeta=\eta$. Definindo $v=\xi-\eta$, vejamos qual é a Fórmula de Taylor de $H$.

$$
H(\xi)=H(\eta)+H^{\prime}(\eta) \cdot v+\frac{1}{2} H^{\prime \prime}(\eta) \cdot v^{(2)}+\ldots+\frac{1}{(m-|\alpha|-1) !} H^{(m-|\alpha|-1)}(\eta) \cdot v^{(m-|\alpha|-1)}+
$$




$$
+\frac{1}{(m-|\alpha|-1) !} \int_{0}^{1}(1-t)^{m-|\alpha|-1} H^{(m-|\alpha|)}(\eta+t(\xi-\eta)) \cdot v^{(m-|\alpha|)} d t .
$$

Assim existem $C>0$ (independente de $\eta$ ) e $\zeta$ no segmento unindo $\xi$ e $\eta$ tais que:

$$
\left|L_{k|\alpha|+k+|\alpha|, k m+k+m}(\xi, \eta, F)\right| \leq C|\xi-\eta|^{m-|\alpha|}\left|H^{(m-|\alpha|)}(\zeta)\right| .
$$

Existe uma constante $C^{*}>0$ tal que

$$
\left|H^{(m-|\alpha|)}(z)\right| \leq C^{*} \sum_{|\gamma|=m-|\alpha|}\left|D^{\gamma} H(z)\right| .
$$

Pela definição de $H$, cada $D^{\gamma} H(\zeta)$ é uma combinação de polinômios em $\zeta$ e em $\eta$ multiplicados por derivadas de $F$ em $\zeta$ e em $\eta$ de ordens menores ou iguais à $k m+k+m$, uma vez que

$$
\begin{aligned}
k|\alpha|+k+|\alpha|+m-|\alpha| & =m+k+k|\alpha| \\
& \leq m+k+k m .
\end{aligned}
$$

O próximo passo da prova do lema é provar que existem constantes $K, n_{4}>0$ tais que se $f \in$ $\mathcal{C}^{p}\left(\mathbb{R}^{N}\right)$ se anula de ordem $p$ em $\mathcal{N}^{k+1}$, se $\xi, \eta \in \mathcal{N}^{k}$ são como antes e $|\beta|=(|\alpha|+1) k,|\gamma|=(m+1) k$, então

$$
\begin{gathered}
\left|D^{\beta}\left(P(\xi)^{|\alpha|+1}\right)\right|\left|D^{\gamma}\left(P(\eta)^{m+1}\right)\right|\left|R_{m}^{(\alpha)}(\xi ; \eta)\right|=\left|L_{k|\alpha|+k+|\alpha|, k m+k+m}(\xi, \eta, F)\right| \leq \\
K|\xi-\eta|^{m-|\alpha|}(1+|\eta|+|\zeta|)^{n_{4}}\left(\operatorname{dist}\left(\eta, \mathcal{N}^{k+1}\right)+\operatorname{dist}\left(\zeta, \mathcal{N}^{k+1}\right)\right)^{2 \mu^{\prime}}|F|_{p, S_{1}(\xi, \eta)} .
\end{gathered}
$$

Para cada $\gamma \in \mathbb{N}^{N}$ tal que $|\gamma|=m-|\alpha|$, podemos escrever que

$$
\left|D^{\gamma} H(w)\right| \leq \sum_{|\gamma| \leq k m+k+m}\left|Q_{\gamma}(w, \eta) D^{\gamma} F(w)\right|+\sum_{|\lambda| \leq k m+k+m}\left|P_{\lambda}(w, \eta) D^{\lambda} F(\eta)\right| .
$$

Pelo lema 8.0.43 do Apêndice, existem constantes $K, n^{*}>0$ tais que

$$
\begin{aligned}
\left|D^{\gamma} H(\zeta)\right| & \leq K \operatorname{dist}\left(\zeta, \mathcal{N}^{k+1}\right)^{2 \mu^{\prime}}(1+|\zeta|+|\eta|)^{n^{*}}|F|_{p, B_{1}[\zeta]}+ \\
& +K \operatorname{dist}\left(\eta, \mathcal{N}^{k+1}\right)^{2 \mu^{\prime}}(1+|\zeta|+|\eta|)^{n^{*}}|F|_{p, B_{1}[\eta]} \\
& \leq K(1+|\zeta|+|\eta|)^{n^{*}}|F|_{p, S(\zeta, \eta)}\left(\operatorname{dist}\left(\zeta, \mathcal{N}^{k+1}\right)^{2 \mu^{\prime}}+\operatorname{dist}\left(\eta, \mathcal{N}^{k+1}\right)^{2 \mu^{\prime}}\right) \\
& \leq K(1+|\zeta|+|\eta|)^{n^{*}}|F|_{p, S(\zeta, \eta)}\left(\operatorname{dist}\left(\zeta, \mathcal{N}^{k+1}\right)+\operatorname{dist}\left(\eta, \mathcal{N}^{k+1}\right)\right)^{2 \mu^{\prime}}
\end{aligned}
$$

Conseqüentemente, existem constantes positivas $C^{\prime}>0, n_{4}$ tais que

$$
\left|H^{(m-|\alpha|)}(\zeta)\right| \leq C^{\prime}(1+|\zeta|+|\eta|)^{n_{4}}|F|_{p, S(\zeta, \eta)}\left(\operatorname{dist}\left(\zeta, \mathcal{N}^{k+1}\right)+\operatorname{dist}\left(\eta, \mathcal{N}^{k+1}\right)\right)^{2 \mu^{\prime}}
$$

Notemos que por (7.0.6) e (7.0.7),

$$
\begin{aligned}
\left(\operatorname{dist}\left(\zeta, \mathcal{N}^{k+1}\right)+\right. & \left.\operatorname{dist}\left(\eta, \mathcal{N}^{k+1}\right)\right)^{2}= \\
& =\operatorname{dist}\left(\eta, \mathcal{N}^{k+1}\right)^{2}+2 \operatorname{dist}\left(\eta, \mathcal{N}^{k+1}\right) \operatorname{dist}\left(\zeta, \mathcal{N}^{k+1}\right)+\operatorname{dist}\left(\zeta, \mathcal{N}^{k+1}\right)^{2} \\
& \leq 3 \operatorname{dist}\left(\eta, \mathcal{N}^{k+1}\right) \operatorname{dist}\left(\xi, \mathcal{N}^{k+1}\right)+2.3 . \operatorname{dist}\left(\eta, \mathcal{N}^{k+1}\right) \operatorname{dist}\left(\xi, \mathcal{N}^{k+1}\right)+ \\
& +9 \operatorname{dist}\left(\xi, \mathcal{N}^{k+1}\right) \operatorname{dist}\left(\eta, \mathcal{N}^{k+1}\right) \\
& =18 \operatorname{dist}\left(\xi, \mathcal{N}^{k+1}\right) \operatorname{dist}\left(\eta, \mathcal{N}^{k+1}\right) .
\end{aligned}
$$


Além disso,

$$
\begin{aligned}
1+|\eta|+|\zeta| & =1+|\eta|+|(1-t) \xi+t \eta| \\
& \leq 1+2|\eta|+|\xi| \\
& \leq 2(1+|\xi|+|\eta|) .
\end{aligned}
$$

Utilizando essas estimativas em (7.0.10) teremos que:

$$
\begin{gathered}
\left|D^{\beta}\left(P(\xi)^{|\alpha|+1}\right)\right|\left|D^{\gamma}\left(P(\eta)^{m+1}\right)\right|\left|R_{m}^{(\alpha)}(\xi ; \eta)\right| \leq \\
K^{\prime}|\xi-\eta|^{m-|\alpha|}\left(\operatorname{dist}\left(\xi, \mathcal{N}^{k+1}\right) \operatorname{dist}\left(\eta, \mathcal{N}^{k+1}\right)\right)^{\mu^{\prime}}(1+|\xi|+|\eta|)^{n_{4}}|F|_{p, S_{1}(\xi, \eta)} .
\end{gathered}
$$

Elevando ao quadrado, somando para todos os $\beta \in \mathbb{N}^{N}$ tais que $|\beta|=(|\alpha|+1) k$, em seguida para os $\gamma \in \mathbb{N}^{N}$ tais que $|\gamma|=(m+1) k$ e tirando a raíz quadrada, teremos, para alguma constante $M$, que

$$
\begin{gathered}
\left|R_{m}^{(\alpha)}(\xi ; \eta)\right| \sqrt{\sum_{\beta}\left|D^{\beta}\left(P(\xi)^{|\alpha|+1}\right)\right|^{2} \sum_{\gamma}\left|D^{\gamma}\left(P(\eta)^{m+1}\right)\right|^{2}} \leq \\
M|\xi-\eta|^{m-|\alpha|}\left(\operatorname{dist}\left(\xi, \mathcal{N}^{k+1}\right) \operatorname{dist}\left(\eta, \mathcal{N}^{k+1}\right)\right)^{\mu^{\prime}}(1+|\xi|+|\eta|)^{n_{4}}|F|_{p, S_{1}(\xi, \eta)} .
\end{gathered}
$$

Sabemos que

$$
\begin{aligned}
& \sqrt{\sum_{\beta}\left|D^{\beta}\left(P(\xi)^{|\alpha|+1}\right)\right|^{2}} \geq \sqrt{c} \operatorname{dist}\left(\xi, \mathcal{N}^{k+1}\right)^{\mu^{\prime}}\left(1+|\xi|^{2}\right)^{\frac{-\mu^{\prime \prime}}{2}}, \forall \xi \in \mathcal{N}^{k}, \\
& \sqrt{\sum_{\gamma}\left|D^{\gamma}\left(P(\eta)^{m+1}\right)\right|^{2}} \geq \sqrt{c} \operatorname{dist}\left(\eta, \mathcal{N}^{k+1}\right)^{\mu^{\prime}}\left(1+|\eta|^{2}\right)^{\frac{-\mu^{\prime \prime}}{2}}, \forall \eta \in \mathcal{N}^{k}
\end{aligned}
$$

e portanto

$$
\begin{aligned}
\left|R_{m}^{(\alpha)}(\xi ; \eta)\right| & \leq \frac{M}{c}(1+|\xi|+|\eta|)^{n_{4}}|\xi-\eta|^{m-|\alpha|}\left(1+|\xi|^{2}\right)^{\frac{\mu^{\prime \prime}}{2}}\left(1+|\eta|^{2}\right)^{\frac{\mu^{\prime \prime}}{2}}|F|_{p, S_{1}(\xi, \eta)} \\
& \leq \frac{M}{c}(1+|\xi|+|\eta|)^{n_{4}}|\xi-\eta|^{m-|\alpha|}(1+|\xi|)^{\mu^{\prime \prime}}(1+|\eta|)^{\mu^{\prime \prime}}|F|_{p, S_{1}(\xi, \eta)} \\
& \leq \frac{M}{c}(1+|\xi|+|\eta|)^{n_{4}}|\xi-\eta|^{m-|\alpha|}(1+|\xi|+|\eta|)^{2 \mu^{\prime \prime}}|F|_{p, S_{1}(\xi, \eta)} \\
& =\frac{M}{c}(1+|\xi|+|\eta|)^{n_{4}+2 \mu^{\prime \prime}}|\xi-\eta|^{m-|\alpha|}|F|_{p, S_{1}(\xi, \eta)}
\end{aligned}
$$

Assim sendo, existem constantes positivas $K^{\prime \prime \prime}$ e $n^{\prime \prime \prime}$ tais que se $f \in \mathcal{C}^{p}\left(\mathbb{R}^{N}\right)$ se anula de ordem $p$ em $\mathcal{N}^{k+1}$, e $\xi, \eta \in \mathcal{N}^{k}$ são tais que

$$
2|\xi-\eta|<\operatorname{dist}\left(\xi, \mathcal{N}^{k+1}\right)+\operatorname{dist}\left(\eta, \mathcal{N}^{k+1}\right),
$$

então

$$
\left|R_{m}^{(\alpha)}(\xi ; \eta)\right| \leq K^{\prime \prime \prime}|\xi-\eta|^{m-|\alpha|}(1+|\xi|+|\eta|)^{n^{\prime \prime \prime}}|F|_{p, S_{1}(\xi, \eta)}, \forall \alpha \in \mathbb{N}^{N}:|\alpha| \leq m
$$


Finalmente podemos definir $m^{\prime}:$ tome $m^{\prime} \in \mathbb{N}$ tal que

$$
2 \mu^{\prime}+2 k m+2 k+2 m \leq m^{\prime} .
$$

Lema 7.0.34 Existem $G>0, p>0$ tais que se $f \in \mathcal{C}^{m^{\prime}}\left(\mathbb{R}^{N}\right)$ se anula de ordem $m^{\prime}$ em $\mathcal{N}^{k+1}$, então

$$
(1+|\xi|)^{n}\left|f^{(\alpha)}(\eta)\right| \leq G \sup _{z \in \mathbb{R}^{N}}(1+|z|)^{p}|F|_{m^{\prime}, z}, \forall \xi \in \mathbb{R}^{N}, \forall \eta \in \mathcal{N}^{k} \cap B_{R}[\xi], \forall \alpha \in \mathbb{N}^{N}:|\alpha| \leq m .
$$

Demonstração: Pelo lema 7.0.30 existem constantes $K, n^{\prime}>0$ tais que se $f \in \mathcal{C}^{m^{\prime}}\left(\mathbb{R}^{N}\right)$ se anula de ordem $m^{\prime}$ em $\mathcal{N}^{k+1}$, então

$$
\left|f^{(\alpha)}(\eta)\right| \leq K(1+|\eta|)^{n^{\prime}}|F|_{m^{\prime}, B_{1}[\eta]}, \forall \eta \in \mathcal{N}^{k}, \forall \alpha \in \mathbb{N}^{N}:|\alpha| \leq m .
$$

Sejam $\xi \in \mathbb{R}^{N}$ e $\eta \in \mathcal{N}^{k} \cap B_{R}[\xi]$. Pelo Corolário 8.0.5 do Apêndice segue que

$$
\begin{aligned}
(1+|\xi|)^{n}\left|f^{(\alpha)}(\eta)\right| \leq & K(1+|\xi|)^{n}(1+|\eta|)^{n^{\prime}}|F|_{m^{\prime}, B_{1}[\eta]} \\
& \leq K^{\prime}(1+|\xi|)^{n}(1+|\eta|)^{n^{\prime}} \sup _{|\beta| \leq m^{\prime}, w \in B_{1}[\eta]}\left|D^{\beta} F(w)\right|
\end{aligned}
$$

Dado $w \in B_{1}[\eta]$,

$$
1+|\eta| \leq 1+|\eta-w|+|w| \leq 1+1+|w| \leq 2(1+|w|) .
$$

Analogamente, se $w \in B_{1}[\eta]$ e $|\eta-\xi| \leq R$,

$$
\begin{aligned}
1+|\xi| & \leq 1+|\xi-w|+|w| \leq 1+|\xi-\eta|+|\eta-w|+|w| \leq 1+R+1+|w| \\
& \leq(R+2)(1+|w|) .
\end{aligned}
$$

Assim,

$$
\begin{aligned}
(1+|\xi|)^{n}|| f^{(\alpha)}(\eta) \mid & \\
& \leq K^{\prime} \sup _{|\beta| \leq m^{\prime}, w \in B_{1}[\eta]}(1+|\xi|)^{n}(1+|\eta|)^{n^{\prime}}\left|D^{\beta} F(w)\right| \\
& =K^{\prime} \sup _{|\beta| \leq m^{\prime}, w \in B_{1}[\eta]}\left(\frac{1+|\xi|}{1+|w|}\right)^{n}(1+|w|)^{n}\left(\frac{1+|\eta|}{1+|w|}\right)^{n^{\prime}}(1+|w|)^{n^{\prime}}\left|D^{\beta} F(w)\right| \\
& \leq K^{\prime \prime} \sup _{|\beta| \leq m^{\prime}, w \in B_{1}[\eta]}(1+|w|)^{n+n^{\prime}}\left|D^{\beta} F(w)\right| \\
& \leq K^{\prime \prime} \sup _{z \in \mathbb{R}^{N}}(1+|z|)^{n+n^{\prime}}|F|_{m^{\prime}, z} \text {. }
\end{aligned}
$$

Lema 7.0.35 Existem constantes positivas $A$ e q tais que se $f \in \mathcal{C}^{m^{\prime}}\left(\mathbb{R}^{N}\right)$ se anula de ordem $m^{\prime}$ em $\mathcal{N}^{k+1}$, se $\xi \in \mathbb{R}^{N}, x, y \in \mathcal{N}^{k} \cap B_{R}[\xi]$ são tais que $x \neq y$ e $2|x-y|<\operatorname{dist}\left(x, \mathcal{N}^{k+1}\right)+$ $\operatorname{dist}\left(y, \mathcal{N}^{k+1}\right)$, então

$$
(1+|\xi|)^{n} \frac{\left|R_{m}^{(\alpha)}(x ; y)\right|}{|x-y|^{m-|\alpha|}} \leq A \sup _{w \in \mathbb{R}^{N}}(1+|w|)^{q}|F|_{m^{\prime}, w}, \forall \alpha \in \mathbb{N}^{N}:|\alpha| \leq m .
$$


Demonstração: Pelo lema 7.0.33 existem constantes $K^{\prime \prime \prime}, n^{\prime \prime \prime}>0$ tais que se $f \in \mathcal{C}^{m^{\prime}}\left(\mathbb{R}^{N}\right)$ se anula de ordem $m^{\prime}$ em $\mathcal{N}^{k+1}$, e se $x, y \in \mathcal{N}^{k}$ são distintos e tais que

$$
2|x-y|<\operatorname{dist}\left(x, \mathcal{N}^{k+1}\right)+\operatorname{dist}\left(y, \mathcal{N}^{k+1}\right),
$$

então

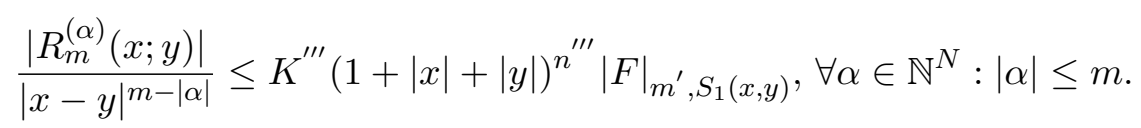

Fixemos $\xi \in \mathbb{R}^{N}$. Como $S_{1}(x, y)$ é convexo, podemos aplicar o Corolário 8.0.5 do Apêndice, e podemos afirmar que existe uma constante $S>0$ (independente de $f$, de $x$, de $y$ e de $\xi$ ) tal que

$$
\begin{aligned}
(1+|\xi|)^{n} \frac{\left|R_{m}^{(\alpha)}(x ; y)\right|}{|x-y|^{m-|\alpha|}} & \leq K^{\prime \prime \prime}(1+|\xi|)^{n}(1+|x|+|y|)^{n^{\prime \prime \prime}}|F|_{m^{\prime}, S_{1}(x, y)} \\
& \leq S . K^{\prime \prime \prime}(1+|\xi|)^{n}(1+|x|+|y|)^{n^{\prime \prime \prime}} \sup _{|\beta| \leq m^{\prime}, w \in S_{1}(x, y)}\left|D^{\beta} F(w)\right| \\
& =S . K^{\prime \prime \prime} \sup _{|\beta| \leq m^{\prime}, w \in S_{1}(x, y)}\left|D^{\beta} F(w)\right|(1+|\xi|)^{n}(1+|x|+|y|)^{n^{\prime \prime \prime}} .
\end{aligned}
$$

Vamos adicionar agora a hipótese que $x, y \in \mathcal{N}^{k} \cap B_{R}[\xi]$. Dado $w \in S_{1}(x, y)$, existe $z \in[x, y]$ tal que $w \in B_{1}[z]$, e portanto

$$
|x-w| \leq|x-z|+|z-w| \leq 2 R+1 \text {. }
$$

Assim,

$$
\begin{aligned}
1+|\xi| & \leq 1+|\xi-w|+|w| \leq 1+|\xi-x|+|x-w|+|w| \leq 1+R+2 R+1+|w| \\
& \leq(2+3 R)(1+|w|)
\end{aligned}
$$

Analogamente,

$$
\begin{aligned}
1+|x|+|y| & \leq 1+|x-w|+|w|+|y-w|+|w| \\
& \leq 1+4 R+2+2|w| \\
& \leq(3+4 R)(1+|w|) .
\end{aligned}
$$

Podemos então concluir que existe uma constante $A$ independente de $\xi$, de $x$ e de $y$ tal que

$$
\begin{aligned}
(1+|\xi|)^{n} \frac{\left|R_{m}^{(\alpha)}(x ; y)\right|}{|x-y|^{m-|\alpha|}} & \leq A \sup _{|\beta| \leq m^{\prime}, w \in S_{1}(x, y)}\left|D^{\beta} F(w)\right|(1+|w|)^{n+n^{\prime \prime \prime}} \\
& \leq A \sup _{w \in \mathbb{R}^{N}}(1+|w|)^{n+n^{\prime \prime \prime}}|F|_{m^{\prime}, w} .
\end{aligned}
$$

Lema 7.0.36 Existem constantes positivas $q^{\prime}$ e $C$ tais que se $f \in \mathcal{C}^{m^{\prime}}\left(\mathbb{R}^{N}\right)$ se anula de ordem $m^{\prime}$ em $\mathcal{N}^{k+1}$, se $\xi \in \mathbb{R}^{N}, x, y \in \mathcal{N}^{k} \cap B_{R}[\xi], x \neq y$, e $2|x-y| \geq \operatorname{dist}\left(x, \mathcal{N}^{k+1}\right)+\operatorname{dist}\left(y, \mathcal{N}^{k+1}\right)$, então

$$
(1+|\xi|)^{n} \frac{\left|R_{m}^{(\alpha)}(x ; y)\right|}{|x-y|^{m-|\alpha|}} \leq C \sup _{w \in \mathbb{R}^{N}}(1+|w|)^{q^{\prime}}|F|_{m^{\prime}, w}, \forall \alpha \in \mathbb{N}^{N}:|\alpha| \leq m .
$$


Demonstração: Pelo lema 7.0.32, existem constantes positivas $K^{\prime \prime}$ e $n^{\prime \prime}$ tais que se $f \in \mathcal{C}^{m^{\prime}}\left(\mathbb{R}^{N}\right)$ se anula de ordem $m^{\prime}$ em $\mathcal{N}^{k+1}$ e $x, y \in \mathcal{N}^{k}$ são tais que

$$
\operatorname{dist}\left(x, \mathcal{N}^{k+1}\right)+\operatorname{dist}\left(y, \mathcal{N}^{k+1}\right) \leq 2|x-y|,
$$

então

$$
\left|R_{m}^{(\alpha)}(x ; y)\right| \leq K^{\prime \prime}(1+|x|+|y|)^{n^{\prime \prime}}|x-y|^{m}|P f|_{m^{\prime}, S(x, y)}, \forall \alpha \in \mathbb{N}^{N}:|\alpha| \leq m .
$$

Assim, dados $\xi \in \mathbb{R}^{N}$ e $x, y$ como antes tais que $x, y \in \mathcal{N}^{k} \cap B_{R}[\xi]$,

$$
(1+|\xi|)^{n}\left|R_{m}^{(\alpha)}(x ; y)\right| \leq K^{\prime \prime}(1+|\xi|)^{n}(1+|x|+|y|)^{n^{\prime \prime}}|x-y|^{m}|F|_{m^{\prime}, S(x, y)} .
$$

Seja $t \in S(x, y)$. Suponhamos, sem perda de generalidade, que $t \in B_{1}[x]$. Então

$$
|t-\xi| \leq|t-x|+|x-\xi| \leq 1+R,
$$

e portanto $S(x, y) \subseteq B_{R+1}[\xi]$. Dessa forma,

$$
\begin{aligned}
(1+|\xi|)^{n} \frac{\left|R_{m}^{(\alpha)}(x ; y)\right|}{|x-y|^{m-|\alpha|}} & \leq K^{\prime \prime}(1+|\xi|)^{n}(1+|x|+|y|)^{n^{\prime \prime}}|x-y|^{|\alpha|}|F|_{m^{\prime}, B_{R+1}[\xi]} \\
& \leq K^{\prime \prime} . S(1+|\xi|)^{n}(1+|x|+|y|)^{n^{\prime \prime}}(2 R)^{|\alpha|} \sup _{|\beta| \leq m^{\prime}, t \in B_{R+1}[\xi]}\left|D^{\beta} F(t)\right| \\
& \leq K^{\prime \prime} . S(2 R)^{m} \sup _{|\beta| \leq m^{\prime}, t \in B_{R+1}[\xi]}(1+|\xi|)^{n}(1+|x|+|y|)^{n^{\prime \prime}}\left|D^{\beta} F(t)\right| .
\end{aligned}
$$

Dado $t \in B_{R+1}[\xi]$,

$$
\begin{aligned}
1+|\xi| & \leq 1+|\xi-t|+|t| \leq 1+R+1+|t|=2+R+|t| \\
& \leq(2+R)(|t|+1) .
\end{aligned}
$$

Além disso,

$$
|x-t| \leq|x-\xi|+|\xi-t| \leq R+R+1=2 R+1,
$$

e portanto

$$
\begin{aligned}
1+|x|+|y| & \leq 1+|x-t|+|t|+|y-t|+|t| \\
& \leq 3+4 R+2|t| \\
& \leq(3+4 R)(1+|t|)
\end{aligned}
$$

Assim,

$$
(1+|\xi|)^{n} \frac{\left|R_{m}^{(\alpha)}(x ; y)\right|}{|x-y|^{m-|\alpha|}} \leq C \sup _{w \in \mathbb{R}^{N}}(1+|w|)^{n+n^{\prime \prime}}|F|_{m^{\prime}, w}
$$

Lema 7.0.37 Supondo que $\mathcal{N}^{k+1} \neq \emptyset$, dados $n, m \in \mathbb{N}$, existem constantes $n^{\prime} \in \mathbb{N}$ e $K>0$ tais que para toda $f \in \mathcal{C}^{m^{\prime}}\left(\mathbb{R}^{N}\right)$ que se anula de ordem $m^{\prime}$ em $\mathcal{N}^{k+1}$,

$$
\sup _{\xi \in \mathbb{R}^{N}}(1+|\xi|)^{n}|f|_{m,\left(\mathcal{N}^{k}\right) \xi} \leq K \sup _{\xi \in \mathbb{R}^{N}}(1+|\xi|)^{n^{\prime}}|P f|_{m^{\prime}, \xi}
$$


Demonstração: Dado $\xi \in \mathbb{R}^{N}$, sejam

$$
\begin{gathered}
A=\left\{(1+|\xi|)^{n}\left|D^{\alpha} f(x)\right|: x \in \mathcal{N}^{k} \cap B_{R}[\xi],|\alpha| \leq m\right\} \\
B=\left\{(1+|\xi|)^{n} \frac{\left|R_{m}^{(\alpha)}(x ; y)\right|}{|x-y|^{m-|\alpha|}}: x, y, \in \mathcal{N}^{k} \cap B_{R}[\xi], x \neq y,|\alpha| \leq m\right\}
\end{gathered}
$$

Temos que

$$
(1+|\xi|)^{n}|f|_{m,\left(\mathcal{N}^{k}\right) \xi}=\sup A \cup B
$$

Pelos últimos três lemas sabemos que existem constantes $K>0$ e $n^{\prime} \in \mathbb{N}$ tais que se $f \in \mathcal{C}^{m^{\prime}}\left(\mathbb{R}^{N}\right)$ se anula de ordem $m^{\prime}$ em $\mathcal{N}^{k+1}$, então

$$
(1+|\xi|)^{n}|f|_{m,\left(\mathcal{N}^{k}\right) \xi} \leq K \sup _{w \in \mathbb{R}^{N}}(1+|w|)^{n^{\prime}}|F|_{m^{\prime}, w}, \forall \xi \in \mathbb{R}^{N}
$$

e portanto

$$
\sup _{\xi \in \mathbb{R}^{N}}(1+|\xi|)^{n}|f|_{m,\left(\mathcal{N}^{k}\right) \xi} \leq K \sup _{w \in \mathbb{R}^{N}}(1+|w|)^{n^{\prime}}|F|_{m^{\prime}, w}
$$

Resta provar o lema acima se $\mathcal{N}^{k+1}=\emptyset$. A prova é muito parecida (mas mais simples) à feita anteriormente.

Lema 7.0.38 Se $\mathcal{N}^{k+1}=\emptyset$ e $n, m \in \mathbb{N}$, existem constantes $n^{\prime} \in \mathbb{N}$ e $K>0$ tais que

$$
\sup _{\xi \in \mathbb{R}^{N}}(1+|\xi|)^{n}|g|_{m,\left(\mathcal{N}^{k}\right) \xi} \leq K \sup _{\xi \in \mathbb{R}^{N}}(1+|\xi|)^{n^{\prime}}|P g|_{m^{\prime}, \xi}, \forall g \in \mathcal{C}^{m^{\prime}}\left(\mathbb{R}^{N}\right) .
$$

Demonstração: Dado $j \in \mathbb{N}$, seja

$$
Q_{j}(\xi)=\sum_{|\beta| \leq j k}\left|D^{\beta}\left(P(\xi)^{j}\right)\right|^{2}
$$

Como vimos, o conjunto de zeros de $Q_{j}$ é $\mathcal{N}^{k+1}$. Dessa forma, o teorema 5.0.13 nos permite afirmar que existem constantes $C_{j}, a_{j}>0$ tais que

$$
\frac{C_{j}}{\left(1+|\xi|^{2}\right)^{a_{j}}} \leq\left|Q_{j}(\xi)\right|, \forall \xi \in \mathbb{R}^{N}
$$

Sejam $c=\min \left\{C_{j}: 1 \leq j \leq m+1\right\}$ e $\mu^{\prime \prime}=\max \left\{a_{j}: 1 \leq j \leq m+1\right\}$. Assim

$$
\frac{c}{\left(1+|\xi|^{2}\right)^{\mu^{\prime \prime}}} \leq\left|Q_{j}(\xi)\right|=\sum_{|\beta| \leq j k}\left|D^{\beta}\left(P(\xi)^{j}\right)\right|^{2}, \forall \xi \in \mathbb{R}^{N}, \forall 1 \leq j \leq m+1
$$

Em particular, como as derivadas de $P^{j}$ de ordens menores que $j k$ se anulam em $\mathcal{N}^{k}$, então

$$
\frac{c}{\left(1+|\xi|^{2}\right)^{\mu^{\prime \prime}}} \leq \sum_{|\beta|=j k}\left|D^{\beta}\left(P(\xi)^{j}\right)\right|^{2}, \forall \xi \in \mathcal{N}^{k}, \forall 1 \leq j \leq m+1 .
$$


Seja, como antes, $F(\xi)=P(\xi) f(\xi)$. Vejamos que existem constantes $n^{\prime}, K>0$ tais que

$$
\left|f^{(\alpha)}(\xi)\right| \leq K(1+|\xi|)^{n^{\prime}}|F|_{m^{\prime}, B_{1}[\xi]}, \forall \xi \in \mathcal{N}^{k}, \forall \alpha \in \mathbb{N}^{N}:|\alpha| \leq m
$$

Fixemos $\alpha \in \mathbb{N}^{N}$ tal que $|\alpha| \leq m$. Na prova do lema 8.0.42 do Apêndice mostramos que existem constantes $K_{1}, n_{1}>0$ tais que

$$
\left|L_{k|\alpha|+k+|\alpha|}^{\beta}(\xi, F)\right| \leq K_{1}(1+|\xi|)^{n_{1}}|F|_{m^{\prime}, B_{1}[\xi]}, \forall \xi \in \mathbb{R}^{N}, \forall \beta \in \mathbb{N}^{N}:|\beta|=(|\alpha|+1) k .
$$

Pelo mesmo argumento feito anteriormente, existe uma constante $K_{2}>0$ tal que

$$
\begin{aligned}
\sqrt{\sum_{|\beta|=(|\alpha|+1) k}\left|L_{k|\alpha|+k+|\alpha|}^{\beta}(\xi, F)\right|^{2}} & =\left\{\sum_{|\beta|=(|\alpha|+1) k}\left|D^{\beta}(P(\xi)|\alpha|+1)\right|^{2}\right\}^{\frac{1}{2}}\left|f^{(\alpha)}(\xi)\right| \\
& \leq K_{2}(1+|\xi|)^{n_{1}}|F|_{m^{\prime}, B_{1}[\xi]}, \forall \xi \in \mathcal{N}^{k} .
\end{aligned}
$$

Pela equação acima e por (7.0.12) concluimos que existem constantes $n_{2}, K_{3}>0$ tais que

$$
\left|f^{(\alpha)}(\xi)\right| \leq K_{3}(1+|\xi|)^{n_{2}}|F|_{m^{\prime}, B_{1}[\xi]}, \forall \xi \in \mathcal{N}^{k} .
$$

As constantes acimas dependem de $\alpha$. Tomando seus máximos, quando $|\alpha| \leq m$, obteremos o desejado.

Nos resta estimar os termos

$$
\frac{R_{m}^{(\alpha)}(\xi ; \eta)}{|\xi-\eta|^{m-|\alpha|}}, \text { onde } \xi, \eta \in \mathcal{N}^{k}, \xi \neq \eta, \alpha \in \mathbb{N}^{N}:|\alpha| \leq m
$$

Sejam $\alpha \in \mathbb{N}^{N}$ tal que $|\alpha| \leq m, \beta, \gamma \in \mathbb{N}^{N}$ tais que $|\beta|=(|\alpha|+1) k,|\gamma|=(m+1) k$. Relembremos que $L_{k|\alpha|+k+|\alpha|, k m+k+m}(\xi, \eta, F)=D_{\xi}^{\beta} D_{\eta}^{\gamma} L_{|\alpha|, m}(\xi, \eta, F)$. Para $\eta \in \mathcal{N}^{k}$ fixado, seja

$$
H(\zeta)=L_{k|\alpha|+k+|\alpha|, k m+k+m}(\zeta, \eta, F) .
$$

Vimos que $H$ se anula de ordem $m-|\alpha|$ para $\zeta=\eta$. Seja $v=\xi-\eta$ e vejamos qual é a fórmula de Taylor de $H$.

$$
\begin{gathered}
H(\xi)=H(\eta)+H^{\prime}(\eta) \cdot v+\frac{1}{2} H^{\prime \prime}(\eta) \cdot v^{(2)}+\ldots+\frac{1}{(m-|\alpha|-1) !} H^{(m-|\alpha|-1)}(\eta) \cdot v^{(m-|\alpha|-1)}+ \\
\quad+\frac{1}{(m-|\alpha|-1) !} \int_{0}^{1}(1-t)^{m-|\alpha|-1} H^{(m-|\alpha|)}(\eta+t(\xi-\eta)) \cdot v^{(m-|\alpha|)} d t .
\end{gathered}
$$

Assim existem $\zeta$ no segmento unindo $\xi$ e $\eta$ e uma constante $C>0$ independente de $\eta$ tais que:

$$
\left|L_{k|\alpha|+k+|\alpha|, k m+k+m}(\xi, \eta, F)\right| \leq C|\xi-\eta|^{m-|\alpha|}\left|H^{(m-|\alpha|)}(\zeta)\right| .
$$

Existe uma constante $C^{*}>0$ tal que

$$
\left|H^{(m-|\alpha|)}(z)\right| \leq C^{*} \sum_{|\gamma|=m-|\alpha|}\left|D^{\gamma} H(z)\right|
$$


Cada $D^{\gamma} H(\zeta)$ é uma combinação de polinômios em $\zeta$ e em $\eta$ multiplicados por derivadas de $F$ em $\zeta$ e em $\eta$ de ordens menores ou iguais à $k m+k+m$, uma vez que

$$
k|\alpha|+k+|\alpha|+m-|\alpha|=m+k+k|\alpha| \leq m+k+k m .
$$

Para cada $\gamma \in \mathbb{N}^{N}$ tal que $|\gamma| \leq m-|\alpha|$, podemos escrever que

$$
\left|D^{\gamma} H(w)\right| \leq \sum_{|\kappa| \leq k m+k+m}\left|Q_{\kappa}(w, \eta) D^{\kappa} F(w)\right|+\sum_{|\lambda| \leq k m+k+m}\left|P_{\lambda}(w, \eta) D^{\lambda} F(\eta)\right| .
$$

Pelo mesmo argumento feito anteriormente, existem constantes $C, n_{4}>0$ tais que

$$
\begin{aligned}
\left|H^{(m-|\alpha|)}(\zeta)\right| & \leq C(1+|\zeta|+|\eta|)^{n_{4}}|F|_{m^{\prime}, B_{1}[\zeta]}+ \\
& +C(1+|\zeta|+|\eta|)^{n_{4}}|F|_{m^{\prime}, B_{1}[\eta]} \\
& \leq C(1+|\zeta|+|\eta|)^{n_{4}}|F|_{m^{\prime}, S(\zeta, \eta)} \\
& \leq C(1+|\zeta|+|\eta|)^{n_{4}}|F|_{m^{\prime}, S_{1}(\xi, \eta)} .
\end{aligned}
$$

Além disso, como vimos, $1+|\eta|+|\zeta| \leq 2(1+|\xi|+|\eta|)$. Assim obtemos que se $\xi, \eta \in \mathcal{N}^{k}$,

$$
\begin{aligned}
L_{k|\alpha|+k+|\alpha|, k m+k+m}(\xi, \eta, F) & =\left|D^{\beta}\left(P(\xi)^{|\alpha|+1}\right)\right|\left|D^{\gamma}\left(P(\eta)^{m+1}\right)\right|\left|R_{m}^{(\alpha)}(\xi ; \eta)\right| \\
& \leq K^{\prime}|\xi-\eta|^{m-|\alpha|}(1+|\xi|+|\eta|)^{n_{4}}|F|_{m^{\prime}, S_{1}(\xi, \eta)} .
\end{aligned}
$$

Elevando ao quadrado, somando para todos os $\beta \in \mathbb{N}^{N}$ tais que $|\beta|=(|\alpha|+1) k$, em seguinda para os $\gamma \in \mathbb{N}^{N}$ tais que $|\gamma|=(m+1) k$ e tirando a raíz quadrada, teremos, para alguma constante $M$ que

$$
\left|R_{m}^{(\alpha)}(\xi ; \eta)\right| \sqrt{\sum_{\beta}\left|D^{\beta}\left(P(\xi)^{|\alpha|+1}\right)\right|^{2} \sum_{\gamma}\left|D^{\gamma}\left(P(\eta)^{m+1}\right)\right|^{2}} \leq M|\xi-\eta|^{m-|\alpha|}(1+|\xi|+|\eta|)^{n_{4}}|F|_{m^{\prime}, S_{1}(\xi, \eta)}
$$

Sabemos que

$$
\begin{aligned}
& \sqrt{\sum_{\beta}\left|D^{\beta}\left(P(\xi)^{|\alpha|+1}\right)\right|^{2}} \geq \sqrt{c}\left(1+|\xi|^{2}\right)^{\frac{-\mu^{\prime \prime}}{2}}, \forall \xi \in \mathcal{N}^{k} \\
& \sqrt{\sum_{\gamma}\left|D^{\gamma}\left(P(\eta)^{m+1}\right)\right|^{2}} \geq \sqrt{c}\left(1+|\eta|^{2}\right)^{\frac{-\mu^{\prime \prime}}{2}}, \forall \eta \in \mathcal{N}^{k} .
\end{aligned}
$$

Conseqüentemente,

$$
\begin{aligned}
\left|R_{m}^{(\alpha)}(\xi ; \eta)\right| & \leq \frac{M}{c}(1+|\xi|+|\eta|)^{n_{4}}|\xi-\eta|^{m-|\alpha|}\left(1+|\xi|^{2}\right)^{\frac{\mu^{\prime \prime}}{2}}\left(1+|\eta|^{2}\right)^{\frac{\mu^{\prime \prime}}{2}}|F|_{m^{\prime}, S_{1}(\xi, \eta)} \\
& \leq \frac{M}{c}(1+|\xi|+|\eta|)^{n_{4}}|\xi-\eta|^{m-|\alpha|}(1+|\xi|)^{\mu^{\prime \prime}}(1+|\eta|)^{\mu^{\prime \prime}}|F|_{m^{\prime}, S_{1}(\xi, \eta)} \\
& \leq \frac{M}{c}(1+|\xi|+|\eta|)^{n_{4}}|\xi-\eta|^{m-|\alpha|}(1+|\xi|+|\eta|)^{2 \mu^{\prime \prime}}|F|_{m^{\prime}, S_{1}(\xi, \eta)} \\
& =\frac{M}{c}(1+|\xi|+|\eta|)^{n_{4}+2 \mu^{\prime \prime}}|\xi-\eta|^{m-|\alpha|}|F|_{m^{\prime}, S_{1}(\xi, \eta)}
\end{aligned}
$$


Assim sendo, existem constantes positivas $K^{\prime \prime \prime}$ e $n^{\prime \prime \prime}$ tais que se $f \in \mathcal{C}^{m^{\prime}}\left(\mathbb{R}^{N}\right)$, então

$$
\left|R_{m}^{(\alpha)}(\xi ; \eta)\right| \leq K^{\prime \prime \prime}|\xi-\eta|^{m-|\alpha|}(1+|\xi|+|\eta|)^{n^{\prime \prime \prime}}|F|_{m^{\prime}, S_{1}(\xi, \eta)}, \forall \xi, \eta \in \mathcal{N}^{k}, \forall \alpha \in \mathbb{N}^{N}:|\alpha| \leq m .
$$

Pelo mesmo argumento do caso $\mathcal{N}^{k+1} \neq \emptyset$ terminamos a prova.

Tendo feito tudo isso poderíamos retornar ao lema 7.0.27 e nos perguntarmos se era realmente necessário encontrar estimativas para

$$
\frac{R_{m}^{(\alpha)}(\xi, \eta)}{|\xi-\eta|^{m-|\alpha|}}
$$

uma vez que já sabemos que existem constantes $n^{\prime}, K>0$ tais que se $f \in \mathcal{C}^{m^{\prime}}\left(\mathbb{R}^{N}\right)$ se anula de ordem $m^{\prime}$ em $\mathcal{N}^{k+1}$,

$$
\left|f^{(\alpha)}(\xi)\right| \leq K(1+|\xi|)^{n^{\prime}}|F|_{m^{\prime}, B_{1}[\xi]}, \forall \xi \in \mathcal{N}^{k}, \forall \alpha \in \mathbb{N}^{N}:|\alpha| \leq m
$$

Vejamos onde está a dificuldade: Pelo lema 7.0.27 existe uma constante $C^{*}>0$ tal que se $\xi \in \mathbb{R}^{N}$, e se $f \in \mathcal{C}^{m^{\prime}}\left(\mathbb{R}^{N}\right)$,

$$
(1+|\xi|)^{n}|f|_{m,\left(\mathcal{N}^{k}\right)_{\xi}} \leq C^{*} \sup _{y \in \mathbb{R}^{N}}(1+|y|)^{n}|f|_{m, y} .
$$

Poderíamos pensar em utilizar (7.0.13), mas precisaríamos de uma estimativa em todo $\mathbb{R}^{N}$, e não só para $\mathcal{N}^{k}$.

Concluiremos agora a demonstração do lema 7.0.26. Relembrando nossos passos, estamos fazendo a prova por indução em $k$ (para $k$ decrescente). Assumimos que o lema é verdadeiro com $k+1$ e estamos provando para $k$. Nessa etapa acrescentamos a hipótese adicional de que $f$ se anula de ordem $m^{\prime}$ em $\mathcal{N}^{k+1}$, onde $m^{\prime}$ foi definido acima. Vimos que nesse caso o lema é verdadeiro; falta, então, provar o lema sem a hipótese adicional de que $f$ se anula de ordem $m^{\prime}$ em $\mathcal{N}^{k+1}$. Seja $f \in \mathcal{C}^{m}\left(\mathbb{R}^{N}\right)$ qualquer.

Utilizaremos o Teorema da Extensão de Whitney com $A=\mathcal{N}^{k+1}$ e com $m$ substituido por $m^{\prime}$. Assim, existe uma função $g \in \mathcal{C}^{m^{\prime}}\left(\mathbb{R}^{N}\right)$ tal que $D^{\alpha} g(\xi)=D^{\alpha} f(\xi), \forall \xi \in \mathcal{N}^{k+1}, \forall \alpha \in \mathbb{N}^{N}$ tal que $|\alpha| \leq m^{\prime}$ e além disso existe uma constante $K_{1}$ tal que

$$
|g|_{m^{\prime}, \xi} \leq K_{1}|f|_{m^{\prime},\left(\mathcal{N}^{k+1}\right) \xi}, \forall \xi \in \mathbb{R}^{N} .
$$

Pelo caso que já provamos do lema 7.0.26 existem constantes positivas $K$ e $n^{\prime}$ (que assumiremos ser tal que $\left.n \leq n^{\prime}\right)$ tais que se $\varphi \in \mathcal{C}^{m^{\prime}}\left(\mathbb{R}^{N}\right)$ e $\varphi$ se anula de ordem $m^{\prime}$ em $\mathcal{N}^{k+1}$, então

$$
\sup _{\xi \in \mathbb{R}^{N}}(1+|\xi|)^{n}|\varphi|_{m,\left(\mathcal{N}^{k}\right) \xi} \leq K \sup _{\xi \in \mathbb{R}^{N}}(1+|\xi|)^{n^{\prime}}|P \varphi|_{m^{\prime}, \xi}
$$

Seja $h=f-g$. Então $h \in \mathcal{C}^{m^{\prime}}\left(\mathbb{R}^{N}\right)$ e $h=0$ de ordem $m^{\prime}+1$ em $\mathcal{N}^{k+1}$. Como $P$ tem grau $\mu$, temos:

$$
\sup _{\xi \in \mathbb{R}^{N}}(1+|\xi|)^{n^{\prime}}|P g|_{m^{\prime}, \xi} \leq K_{2} \sup _{\xi \in \mathbb{R}^{N}}(1+|\xi|)^{n^{\prime}+\mu}|g|_{m^{\prime}, \xi}
$$


Mas então por (7.0.14) e (7.0.15),

$$
\sup _{\xi \in \mathbb{R}^{N}}(1+|\xi|)^{n^{\prime}}|P g|_{m^{\prime}, \xi} \leq K_{3} \sup _{\xi \in \mathbb{R}^{N}}(1+|\xi|)^{n^{\prime}+\mu}|f|_{m^{\prime},\left(\mathcal{N}^{k+1}\right) \xi} .
$$

Pela hipótese de indução, existem naturais $n^{\prime \prime}, m^{\prime \prime}$ (que vamos assumir que são tais que $n^{\prime} \leq n^{\prime \prime}$ e $\left.m^{\prime} \leq m^{\prime \prime}\right)$ e $L_{2}>0$ tais que

$$
\sup _{\xi \in \mathbb{R}^{N}}(1+|\xi|)^{n^{\prime}+\mu}|f|_{m^{\prime},\left(\mathcal{N}^{k+1}\right) \xi} \leq L_{2} \sup _{\xi \in \mathbb{R}^{N}}(1+|\xi|)^{n^{\prime \prime}}|P f|_{m^{\prime \prime}, \xi}
$$

Por (7.0.16) existe $K_{3}$ tal que

$$
\sup _{\xi \in \mathbb{R}^{N}}(1+|\xi|)^{n^{\prime}}|P g|_{m^{\prime}, \xi} \leq K_{3} \sup _{\xi \in \mathbb{R}^{N}}(1+|\xi|)^{n^{\prime \prime}}|P f|_{m^{\prime \prime}, \xi}
$$

Como $h=f-g$,

$$
\begin{aligned}
\sup _{\xi \in \mathbb{R}^{N}}(1+|\xi|)^{n^{\prime}}|P h|_{m^{\prime}, \xi} & \leq \sup _{\xi \in \mathbb{R}^{N}}(1+|\xi|)^{n^{\prime}}|P f|_{m^{\prime}, \xi}+\sup _{\xi \in \mathbb{R}^{N}}(1+|\xi|)^{n^{\prime}}|P g|_{m^{\prime}, \xi} \\
& \leq\left(K_{4}+1\right) \sup _{\xi \in \mathbb{R}^{N}}(1+|\xi|)^{n^{\prime \prime}}|P f|_{m^{\prime \prime}, \xi}
\end{aligned}
$$

já que $n^{\prime} \leq n^{\prime \prime}$ e $m^{\prime} \leq m^{\prime \prime}$.

Como $h$ se anula de ordem $m^{\prime}$ em $\mathcal{N}^{k+1}$ podemos aplicar o resultado já provado. Assim, existe uma constante $C>0$ tal que

$$
\begin{aligned}
\sup _{\xi \in \mathbb{R}^{N}}(1+|\xi|)^{n}|h|_{m,\left(\mathcal{N}^{k}\right) \xi} & \leq C \sup _{\xi \in \mathbb{R}^{N}}(1+|\xi|)^{n^{\prime}}|P h|_{m^{\prime}, \xi} \\
& \leq C\left(K_{4}+1\right) \sup _{\xi \in \mathbb{R}^{N}}(1+|\xi|)^{n^{\prime \prime}}|P f|_{m^{\prime \prime}, \xi} .
\end{aligned}
$$

Como $f=h+g$,

$$
\sup _{\xi \in \mathbb{R}^{N}}(1+|\xi|)^{n}|f|_{m,\left(\mathcal{N}^{k}\right) \xi} \leq \sup _{\xi \in \mathbb{R}^{N}}(1+|\xi|)^{n}|h|_{m,\left(\mathcal{N}^{k}\right) \xi}+\sup _{\xi \in \mathbb{R}^{N}}(1+|\xi|)^{n}|g|_{m,\left(\mathcal{N}^{k}\right) \xi}
$$

O lema 7.0.27 nos permite afirmar que existe $C^{*}>0$ tal que

$$
\begin{aligned}
\sup _{\xi \in \mathbb{R}^{N}}(1+|\xi|)^{n}|g|_{m,\left(\mathcal{N}^{k}\right) \xi} & \leq C^{*} \sup _{\xi \in \mathbb{R}^{N}}(1+|\xi|)^{n}|g|_{m, \xi} \\
& \leq C^{*} \sup _{\xi \in \mathbb{R}^{N}}(1+|\xi|)^{n}|g|_{m^{\prime}, \xi}
\end{aligned}
$$

Utilizando então (7.0.14) e (7.0.17) obtemos que

$$
\begin{aligned}
\sup _{\xi \in \mathbb{R}^{N}}(1+|\xi|)^{n}|g|_{m,\left(\mathcal{N}^{k}\right) \xi} & \leq K_{5} \sup _{\xi \in \mathbb{R}^{N}}(1+|\xi|)^{n}|f|_{m^{\prime},\left(\mathcal{N}^{k+1}\right) \xi} \\
& \leq K_{5} \sup _{\xi \in \mathbb{R}^{N}}(1+|\xi|)^{n^{\prime}+\mu}|f|_{m^{\prime},\left(\mathcal{N}^{k+1}\right) \xi} \\
& \leq K_{6} \sup _{\xi \in \mathbb{R}^{N}}(1+|\xi|)^{n^{\prime \prime}}|P f|_{m^{\prime \prime}, \xi},
\end{aligned}
$$


Concluimos finalmente, por (7.0.18) e pelas observações acima, que existe uma constante $K>0$ tal que

$$
\sup _{\xi \in \mathbb{R}^{N}}(1+|\xi|)^{n}|f|_{m,\left(\mathcal{N}^{k}\right) \xi} \leq K \sup _{\xi \in \mathbb{R}^{N}}(1+|\xi|)^{n^{\prime \prime}}|P f|_{m^{\prime \prime}, \xi}
$$




\section{Capítulo 8}

\section{Apêndice}

Lema 8.0.39 As derivadas de $R_{m}^{(\alpha)}(\xi ; \eta)$ com respeito a $\xi$ e a $\eta$ de ordens menores ou iguais a $m-|\alpha|$ se anulam em $\xi=\eta \in \mathcal{N}^{k}$.

Demonstração: Relembrando,

$$
R_{m}^{(\alpha)}(\xi ; \eta)=f^{(\alpha)}(\xi)-\sum_{|\beta| \leq m-|\alpha|} f^{(\alpha+\beta)}(\eta) \frac{(\xi-\eta)^{\beta}}{\beta !} .
$$

Sejam $p, q \in \mathbb{N}^{N}$ com $|p+q| \leq m-|\alpha|$. Temos que

$$
\partial_{\xi}^{p} R_{m}^{(\alpha)}(\xi ; \eta)=f^{(\alpha+p)}(\xi)-\sum_{p \leq \beta ;|\beta| \leq m-|\alpha|} f^{(\alpha+\beta)}(\eta) \frac{(\xi-\eta)^{\beta-p}}{(\beta-p) !} .
$$

Aplicando o operador $\partial_{\eta}^{q}$ na expressão acima obteremos:

$$
\partial_{\eta}^{q} \partial_{\xi}^{p} R_{m}^{(\alpha)}(\xi ; \eta)=-\sum_{p \leq \beta ;|\beta| \leq m-|\alpha|} \sum_{r \leq q, r \leq \beta-p}\left(\begin{array}{l}
q \\
r
\end{array}\right) f^{(\alpha+\beta+q-r)}(\eta) \partial_{\eta}^{r}\left\{\frac{(\xi-\eta)^{\beta-p}}{(\beta-p) !}\right\} .
$$

Fazendo $\xi=\eta \in \mathcal{N}^{k}$ teremos:

$$
\begin{aligned}
\partial_{\eta}^{q} \partial_{\xi}^{p} R_{m}^{(\alpha)}(\eta ; \eta)= & -\sum_{|\beta| \leq m-|\alpha|, p \leq \beta \leq p+q}\left(\begin{array}{c}
q \\
\beta-p
\end{array}\right) f^{(\alpha+q+p)}(\eta)(-1)^{|\beta-p|} \\
& =-f^{(\alpha+p+q)}(\eta) \sum_{p \leq \beta \leq p+q}(-1)^{|\beta-p|}\left(\begin{array}{c}
q \\
\beta-p
\end{array}\right) .
\end{aligned}
$$

Resta ver que

$$
\sum_{p \leq \beta \leq p+q}(-1)^{|\beta-p|}\left(\begin{array}{c}
q \\
\beta-p
\end{array}\right)=0 .
$$

Fazendo $\beta-p=\lambda$ teremos que

$$
\sum_{p \leq \beta \leq p+q}(-1)^{|\beta-p|}\left(\begin{array}{c}
q \\
\beta-p
\end{array}\right)=\sum_{0 \leq \lambda \leq q}(-1)^{|\lambda|}\left(\begin{array}{l}
q \\
\lambda
\end{array}\right) .
$$


Mas

$$
\begin{aligned}
0 & =(1-1)^{q}=(1-1, \ldots, 1-1)^{q} \\
& =(1-1)^{q_{1}} \ldots \ldots(1-1)^{q_{N}} \\
& =\left(\sum_{\gamma_{1}=0}^{q_{1}}\left(\begin{array}{l}
q_{1} \\
\gamma_{1}
\end{array}\right)(-1)^{\gamma_{1}}\right) \ldots\left(\sum_{\gamma_{N}=0}^{q_{N}}\left(\begin{array}{l}
q_{N} \\
\gamma_{N}
\end{array}\right)(-1)^{\gamma_{N}}\right) \\
& =\sum_{\gamma_{1}=0}^{q_{1}} \ldots \sum_{\gamma_{N}=0}^{q_{N}}\left(\begin{array}{c}
q \\
\left(\gamma_{1}, \ldots, \gamma_{N}\right)
\end{array}\right)(-1)^{|\gamma|}=\sum_{\gamma \leq q}\left(\begin{array}{l}
q \\
\gamma
\end{array}\right)(-1)^{|\gamma|} .
\end{aligned}
$$

donde segue o resultado.

Lema 8.0.40 Dado $j \in \mathbb{N}^{*}$, seja

$$
Q_{j}(\xi)=\sum_{|\beta| \leq j k}\left|D^{\beta}\left(P(\xi)^{j}\right)\right|^{2} .
$$

Então $\left\{\xi \in \mathbb{R}^{N}: Q_{j}(\xi)=0\right\}=\mathcal{N}^{k+1}$.

Demonstração: Vejamos inicialmente que $\left\{\xi \in \mathbb{R}^{N}: Q_{j}(\xi)=0\right\} \subseteq \mathcal{N}^{k+1}$. Queremos provar que se $D^{\beta}\left(P^{j}\right)(\xi)=0, \forall \beta \in \mathbb{N}^{N}$ tal que $|\beta| \leq j k$, então $\left(D^{\alpha} P\right)(\xi)=0, \forall \alpha \in \mathbb{N}^{N}$ tal que $|\alpha| \leq k$. Seja $\eta \in \mathbb{R}^{N}$ tal que $D^{\beta} P^{j}(\eta)=0, \forall \beta \in \mathbb{N}^{N}$ tal que $|\beta| \leq j k$. Escrevendo a Fórmula de Taylor de $P^{j}$ em $\eta$ vemos que $P^{j}(\xi)=\mathrm{O}\left(|\xi-\eta|^{j k+1}\right)$. Conseqüentemente,

$$
P(\xi)=\mathrm{O}\left(|\xi-\eta|^{k+\frac{1}{j}}\right)
$$

Disso concluiremos que $D^{\alpha} P(\eta)=0, \forall \alpha \in \mathbb{N}^{N}$ tal que $|\alpha| \leq k$. Suponhamos inicialmente que $\eta=0$. Então

$$
P(\xi)=\sum_{|\alpha| \leq k} D^{\alpha} P(0) \frac{\xi^{\alpha}}{\alpha !}+\mathrm{O}\left(|\xi|^{k+1}\right)=\sum_{l=0}^{k} q_{l}(\xi)+\mathrm{O}\left(|\xi|^{k+1}\right),
$$

onde $q_{l}(\xi)=\sum_{|\beta|=l} D^{\beta} P(0) \frac{\xi^{\beta}}{\beta !}$. Temos que as funções $q_{l}$ são homogêneas de grau $l$. Veremos que as funções $q_{l}$ são nulas. Suponhamos por absurdo que existe algum $l \leq k$ tal que $q_{l}$ não é nula. Sejam $l_{0}$ o menor valor de $l$ tal que $q_{l}$ não é nula e $v \in \mathbb{R}^{N}$ tal que $q_{l_{0}}(v) \neq 0$. Dessa forma, se $t$ for pequeno,

$$
P(t v)=t^{l_{0}}\left(\sum_{l=l_{0}}^{k} t^{l-l_{0}} q_{l}(v)+\frac{\mathrm{O}\left(|t v|^{k+1}\right)}{t^{l_{0}}}\right)=a(t) t^{l_{0}},
$$

onde $a(0) \neq 0$, já que $q_{l_{0}}(v) \neq 0$. Mas temos que

$$
P(t v)=\mathrm{O}\left(|t|^{k+\frac{1}{j}}\right)
$$

o que não é possível. Concluimos que as funções $q_{l}$ são nulas. Como elas são polinômios podemos concluir que $D^{\alpha} P(0)=0, \forall \alpha \in \mathbb{N}^{N}$ tal que $|\alpha| \leq k$.

No caso geral, se $P(\xi)=\mathrm{O}\left(|\xi-\eta|^{k+\frac{1}{j}}\right)$, seja $Q(\xi)=P(\xi+\eta)$. Temos que $Q(\xi)=\mathrm{O}\left(|\xi|^{k+\frac{1}{j}}\right)$, e portanto, pelo caso anterior, $D^{\beta} Q(0)=0, \forall \beta \in \mathbb{N}^{N}$ tal que $|\beta| \leq k$. Mas $D^{\beta} Q(0)=D^{\beta} P(\eta)$. 
Quanto à recíproca, queremos ver que $\mathcal{N}^{k+1} \subseteq\left\{\xi \in \mathbb{R}^{N}: Q_{j}(\xi)=0\right\}$. Seja $\eta \in \mathcal{N}^{k+1}$. Escrevendo a Fórmula de Taylor de $P$ em $\eta$ temos que $P(\xi)=\mathrm{O}\left(|\xi-\eta|^{k+1}\right)$, e portanto $P^{j}(\xi)=$ $\mathrm{O}\left(|\xi-\eta|^{j k+j}\right)$. Concluiremos por um argumento análogo ao do outro lado da igualdade que $D^{\beta} P^{j}(\eta)=0, \forall \beta \in \mathbb{N}^{N}$ tal que $|\beta| \leq j k$. Suponhamos inicialmente que $\eta=0$ e escrevamos, como antes, que

$$
P^{j}(\xi)=\sum_{|\alpha| \leq j k} D^{\alpha}\left(P^{j}\right)(0) \frac{\xi^{\alpha}}{\alpha !}+\mathrm{O}\left(|\xi|^{j k+1}\right)=\sum_{l=0}^{j k} q_{l}(\xi)+\mathrm{O}\left(|\xi|^{j k+1}\right),
$$

onde cada $q_{l}$ é um polinômio e também uma função homogênea de grau $l$. Suponhamos por absurdo que existe $l \leq j k$ tal que $q_{l} \neq 0$ e seja $l_{0}$ o menor valor de $l$ que satisfaz essa propriedade. Seja $v \in \mathbb{R}^{N}$ tal que $q_{l_{0}}(v) \neq 0$. Temos que, para $t$ pequeno,

$$
P^{j}(t v)=t^{l_{0}}\left(\sum_{l=l_{0}}^{j k} t^{l-l_{0}} q_{l}(v)+\frac{\mathrm{O}\left(|t v|^{j k+1}\right)}{t^{l_{0}}}\right)=a(t) t^{l_{0}},
$$

onde $a(0) \neq 0$, uma vez que $q_{l_{0}}(v) \neq 0$. Mas $P^{j}(t v)=\mathrm{O}\left(|t v|^{j k+j}\right)$, o que não é possível. Concluimos então que as funções $q_{l}$ são nulas; como elas são polinômios podemos concluir que $D^{\beta}\left(P^{j}\right)(0)=0, \forall \beta \in \mathbb{N}^{N}$ tal que $|\beta| \leq j k$. No caso geral, se $P^{j}(\xi)=\mathrm{O}\left(|\xi-\eta|^{j k+j}\right)$, seja $Q(\xi)=P^{j}(\xi+\eta)$. Temos que $Q(\xi)=\mathrm{O}\left(|\xi|^{j k+j}\right)$, donde pelo caso anterior $D^{\beta} Q(0)=0, \forall \beta \in \mathbb{N}^{N}$ tal que $|\beta| \leq j k$. Mas $D^{\beta} Q(0)=D^{\beta} P^{j}(\eta)$, e portanto segue o resultado.

Lema 8.0.41 Existem constantes $c, \mu^{\prime}, \mu^{\prime \prime}>0$ tais que

$$
\sum_{|\beta|=j k}\left|D^{\beta}\left(P(\xi)^{j}\right)\right|^{2} \geq c \operatorname{dist}\left(\xi, \mathcal{N}^{k+1}\right)^{2 \mu^{\prime}}\left(1+|\xi|^{2}\right)^{-\mu^{\prime \prime}}, \forall \xi \in \mathcal{N}^{k}, \forall j \leq(m+1) .
$$

Demonstração: Sabemos que para cada $1 \leq j \leq m+1$, existem constantes $c_{j}, a_{j}, b_{j}>0$ tais que

$$
\sum_{|\beta|=j k}\left|D^{\beta}\left(P(\xi)^{j}\right)\right|^{2} \geq c_{j} \operatorname{dist}\left(\xi, \mathcal{N}^{k+1}\right)^{2 a_{j}}\left(1+|\xi|^{2}\right)^{-b_{j}}, \forall \xi \in \mathcal{N}^{k}
$$

Dado $y \in \mathcal{N}^{k+1}$, faça $\lambda=2(1+|y|)$. Dado $\xi \in \mathcal{N}^{k}$, se $|\xi| \leq 1$, então $|\xi| \leq 1+|\xi|^{2}$, e se $1 \leq|\xi|$ então $|\xi| \leq|\xi|^{2} \leq|\xi|^{2}+1$. Assim,

$$
\frac{|\xi|}{1+|\xi|^{2}} \leq 1
$$

Além disso, $|y| / \lambda\left(1+|\xi|^{2}\right) \leq|y| / \lambda$. Com essas desigualdades em mente, seja $\xi \in \mathcal{N}^{k}$. Temos que

$$
\begin{aligned}
\left|\frac{\operatorname{dist}\left(\xi, \mathcal{N}^{k+1}\right)}{\lambda\left(1+|\xi|^{2}\right)}\right| & =\left|\frac{\operatorname{dist}\left(\xi, \mathcal{N}^{k+1}\right)-\operatorname{dist}\left(y, \mathcal{N}^{k+1}\right)}{\lambda\left(1+|\xi|^{2}\right)}\right| \\
& \leq \frac{|\xi-y|}{\lambda\left(1+|\xi|^{2}\right)} \\
& \leq \frac{|\xi|}{\lambda\left(1+|\xi|^{2}\right)}+\frac{|y|}{\lambda\left(1+|\xi|^{2}\right)} \\
& \leq \frac{1}{\lambda}+\frac{|y|}{\lambda} \leq \frac{1}{2}+\frac{1}{2}=1 .
\end{aligned}
$$


Sejam $a=\max \left\{a_{i}: 1 \leq i \leq m+1\right\}$ e $b=\max \left\{b_{i}: 1 \leq i \leq m+1\right\}$ Para $1 \leq j \leq m+1$,

$$
\begin{aligned}
c_{j} \operatorname{dist}\left(\xi, \mathcal{N}^{k+1}\right)^{2 a_{j}}\left(\frac{1}{1+|\xi|^{2}}\right)^{b_{j}} & \geq c_{j} \operatorname{dist}\left(\xi, \mathcal{N}^{k+1}\right)^{2 a_{j}}\left(\frac{1}{1+|\xi|^{2}}\right)^{b} \\
& =c_{j} \lambda^{2 a_{j}}\left(\frac{\operatorname{dist}\left(\xi, \mathcal{N}^{k+1}\right)}{\lambda\left(1+|\xi|^{2}\right)}\right)^{2 a_{j}}\left(\frac{1}{1+|\xi|^{2}}\right)^{b-2 a_{j}} \\
& \geq c_{j} \lambda^{2 a_{j}}\left(\frac{\operatorname{dist}\left(\xi, \mathcal{N}^{k+1}\right)}{\lambda\left(1+|\xi|^{2}\right)}\right)^{2 a}\left(\frac{1}{1+|\xi|^{2}}\right)^{b-2 a_{j}} \\
& =c_{j} \frac{\lambda^{2 a_{j}}}{\lambda^{2 a}} \operatorname{dist}\left(\xi, \mathcal{N}^{k+1}\right)^{2 a}\left(\frac{1}{1+|\xi|^{2}}\right)^{b-2 a_{j}+2 a} .
\end{aligned}
$$

Sejam $c=\min \left\{c_{i} \lambda^{2\left(a_{i}-a\right)}: 1 \leq i \leq m+1\right\}, \mu^{\prime \prime}=b+2 a$ e $\mu^{\prime}=a$,

$$
c_{j} \frac{\lambda^{2 a_{j}}}{\lambda^{2 a}} \operatorname{dist}\left(\xi, \mathcal{N}^{k+1}\right)^{2 a}\left(\frac{1}{1+|\xi|^{2}}\right)^{b-2 a_{j}+2 a} \geq c \operatorname{dist}\left(\xi, \mathcal{N}^{k+1}\right)^{2 \mu^{\prime}}\left(\frac{1}{1+|\xi|^{2}}\right)^{\mu^{\prime \prime}}
$$

donde concluimos que

$$
\sum_{|\beta|=j k}\left|D^{\beta}\left(P(\xi)^{j}\right)\right|^{2} \geq c \operatorname{dist}\left(\xi, \mathcal{N}^{k+1}\right)^{2 \mu^{\prime}}\left(1+|\xi|^{2}\right)^{-\mu^{\prime \prime}}, \forall \xi \in \mathcal{N}^{k}, \forall j \leq(m+1) .
$$

Lema 8.0.42 Seja $r \in \mathbb{N}$ tal que $\mu^{\prime}+k|\alpha|+k+|\alpha| \leq r$. Existem constantes $K_{1}>0, n_{1} \in \mathbb{N}$ tais que se $f \in \mathcal{C}^{r}\left(\mathbb{R}^{N}\right)$ se anula de ordem $r$ em $\mathcal{N}^{k+1}$, e $\beta \in \mathbb{N}^{N}$ é tal que $|\beta|=(|\alpha|+1) k$, então

$$
\left|L_{k|\alpha|+k+|\alpha|}^{\beta}(\xi, F)\right| \leq K_{1}(1+|\xi|)^{n_{1}} \operatorname{dist}\left(\xi, \mathcal{N}^{k+1}\right)^{r-k|\alpha|-k-|\alpha|}|F|_{r, B_{1}[\xi]}, \forall \xi \in \mathbb{R}^{N} .
$$

onde $F(\xi)=P(\xi) f(\xi)$.

Demonstração: Podemos escrever que

$$
L_{k|\alpha|+k+|\alpha|}^{\beta}(\xi, F)=\sum_{|\lambda| \leq k|\alpha|+k+|\alpha|} Q_{\beta, \lambda}(\xi) D^{\lambda} F(\xi),
$$

onde cada $Q_{\beta, \lambda}$ é um polinômio que não depende de $f$. Então existem constantes $C_{\beta, \lambda}>0 \mathrm{e}$ $n_{\beta, \lambda} \in \mathbb{N}$ tais que $\left|Q_{\beta, \lambda}(x)\right| \leq C_{\beta, \lambda}(1+|x|)^{n_{\beta, \lambda}}, \forall x \in \mathbb{R}^{N}$.

Sejam $C_{\beta}=\max \left\{C_{\beta, \lambda}:|\lambda| \leq k|\alpha|+k+|\alpha|\right\}$ e $p_{\beta}=\max \left\{n_{\beta, \lambda}:|\lambda| \leq k|\alpha|+k+|\alpha|\right\}$. Suponhamos inicialmente que $\operatorname{dist}\left(\xi, \mathcal{N}^{k+1}\right) \geq 1$. Então

$$
\begin{aligned}
\left|L_{k|\alpha|+k+|\alpha|}^{\beta}(\xi, F)\right| & \leq \sum_{|\lambda| \leq k|\alpha|+k+|\alpha|}\left|Q_{\beta, \lambda}(\xi) D^{\lambda} F(\xi)\right| \\
& \leq \sum_{|\lambda| \leq k|\alpha|+k+|\alpha|} C_{\beta, \lambda}(1+|\xi|)^{n_{\beta, \lambda} \mid} D^{\lambda} F(\xi) \mid \\
& \leq C_{\beta}(1+|\xi|)^{p_{\beta}} \sum_{|\lambda| \leq k|\alpha|+k+|\alpha|}\left|D^{\lambda} F(\xi)\right| \\
& \leq C_{\beta}(1+|\xi|)^{p_{\beta}} \sum_{|\lambda| \leq r}\left|D^{\lambda} F(\xi)\right| \\
& \leq C_{\beta} \cdot C^{\prime}(1+|\xi|)^{p_{\beta}}|F|_{r, B_{1}[\xi]} \\
& \leq C_{\beta} \cdot C^{\prime}(1+|\xi|)^{p_{\beta}} \operatorname{dist}\left(\xi, \mathcal{N}^{k+1}\right)^{r-k|\alpha|-k-|\alpha|}|F|_{r, B_{1}[\xi]} .
\end{aligned}
$$


Se, ao contrário, $\operatorname{dist}\left(\xi, \mathcal{N}^{k+1}\right)<1$, então existe $\xi^{*} \in \mathcal{N}^{k+1}$ tal que $\left|\xi^{*}-\xi\right|=\operatorname{dist}\left(\xi, \mathcal{N}^{k+1}\right)<1$, e portanto $\xi^{*} \in B_{1}[\xi]$.

Como $f$ se anula de ordem $r$ em $\mathcal{N}^{k+1}$, então $F=P f$ se anula de ordem $r$ em $\xi^{*} \in \mathcal{N}^{k+1}$. Conseqüentemente, a função $L_{k|\alpha|+k+|\alpha|}^{\beta}(\xi, F)$ se anula de ordem $r-k|\alpha|-k-|\alpha|$ em $\xi^{*}$. Para simplificar seja $p=r-k|\alpha|-k-|\alpha|$. Escrevendo a fórmula de Taylor da função $L_{k|\alpha|+k+|\alpha|}^{\beta}(\xi, F)$ no ponto $\xi^{*}$ teremos:

$$
\begin{aligned}
L_{k|\alpha|+k+|\alpha|}^{\beta}(\xi, F) & =\sum_{|\gamma|<p} \frac{1}{\gamma !} \partial^{\gamma} L_{k|\alpha|+k+|\alpha|}^{\beta}\left(\xi^{*}, F\right)\left(\xi-\xi^{*}\right)^{\gamma}+ \\
& +p \int_{0}^{1}(1-t)^{p-1} \sum_{|\gamma|=p} \frac{1}{\gamma !} \partial^{\gamma} L_{k|\alpha|+k+|\alpha|}^{\beta}\left(\xi^{*}+t\left(\xi-\xi^{*}\right)\right)\left(\xi-\xi^{*}\right)^{\gamma} d t .
\end{aligned}
$$

Notemos que $\left|\xi^{*}+t\left(\xi-\xi^{*}\right)-\xi\right|=\left|(t-1)\left(\xi-\xi^{*}\right)\right| \leq\left|\xi-\xi^{*}\right| \leq 1$. Além disso, como $L_{k|\alpha|+k+|\alpha|}^{\beta}(\xi, F)$ se anula em $\xi^{*}$ de ordem $p$, existe uma constante $M>0$ tal que:

$$
\begin{aligned}
\left|L_{k|\alpha|+k+|\alpha|}^{\beta}(\xi, F)\right| & \leq M\left|\xi-\xi^{*}\right|^{p} \sup _{|\gamma|=p} \sup _{\eta \in B_{1}[\xi]}\left|\partial^{\gamma} L_{k|\alpha|+k+|\alpha|}^{\beta}(\eta, F)\right| \\
& =M \operatorname{dist}\left(\xi, \mathcal{N}^{k+1}\right)^{r-k|\alpha|-k-|\alpha|} \sup _{|\gamma|=r-k|\alpha|-k-|\alpha|} \sup _{\eta \in B_{1}[\xi]}\left|\partial^{\gamma} L_{k|\alpha|+k+|\alpha|}^{\beta}(\eta, F)\right| .
\end{aligned}
$$

Se $|\gamma|=r-k|\alpha|-k-|\alpha|$, podemos escrever

$$
\left|\partial^{\gamma} L_{k|\alpha|+k+|\alpha|}^{\beta}(\eta, F)\right|=\left|\sum_{|\lambda| \leq r} R_{\beta, \lambda}(\eta) D^{\lambda} F(\eta)\right|
$$

onde cada $R_{\beta, \lambda}$ é um polinômio (independente de $f$ ). Assim existem constantes $T_{\beta, \gamma}>0, n_{\beta, \gamma} \in \mathbb{N}$ tais que, para $\eta \in B_{1}[\xi]$,

$$
\begin{aligned}
\left|\partial^{\gamma} L_{k|\alpha|+k+|\alpha|}^{\beta}(\eta, F)\right| & \leq T_{\beta, \gamma}(1+|\eta|)^{n_{\beta, \gamma}} \sum_{|\lambda| \leq r}\left|D^{\lambda} F(\eta)\right| \\
& \leq T_{\beta, \gamma}(1+|\eta-\xi|+|\xi|)^{n_{\beta, \gamma}} \sum_{|\lambda| \leq r}\left|D^{\lambda} F(\eta)\right| \\
& \leq T_{\beta, \gamma} 2^{n_{\beta, \gamma}}(1+|\xi|)^{n_{\beta, \gamma}} \sum_{|\lambda| \leq r}\left|D^{\lambda} F(\eta)\right| .
\end{aligned}
$$

Portanto existem constantes $A_{\beta}>0$ e $n_{\beta} \in \mathbb{N}$, independentes de $\xi$, tais que

$$
\sup _{|\gamma|=r-k|\alpha|-k-|\alpha|} \sup _{\eta \in B_{1}[\xi]}\left|\partial^{\gamma} L_{k|\alpha|+k+|\alpha|}^{\beta}(\eta, F)\right| \leq A_{\beta}(1+|\xi|)^{n_{\beta}}|F|_{r, B_{1}[\xi]},
$$

donde existem constantes $B_{\beta}>0$ e $l_{\beta} \in \mathbb{N}$ tais que

$$
\left|L_{k|\alpha|+k+|\alpha|}^{\beta}(\xi, F)\right| \leq B_{\beta}(1+|\xi|)^{l_{\beta}} \operatorname{dist}\left(\xi, \mathcal{N}^{k+1}\right)^{r-k|\alpha|-k-|\alpha|}|F|_{r, S(\xi)}, \forall \xi \in \mathbb{R}^{N} .
$$

Definindo $K_{1}=\max \left\{B_{\beta}:|\beta|=(|\alpha|+1) k\right\}$ e $n_{1}=\max \left\{n_{\beta}:|\beta|=(|\alpha|+1) k\right\}$ segue o resultado. 
Lema 8.0.43 Seja $p \in \mathbb{N}$ tal que $m+k+k m+2 \mu^{\prime} \leq p$. Para cada $\gamma \in \mathbb{N}^{N}$ tal que $|\gamma| \leq k m+k+m$, seja $Q_{\gamma}(w, \eta)$ um polinômio. Existem constantes $C>0, q \in \mathbb{N}$ tais que se $f \in \mathcal{C}^{p}\left(\mathbb{R}^{N}\right)$ se anula de ordem $p$ em $\mathcal{N}^{k+1}$, então,

$$
\left|\sum_{|\gamma| \leq k m+k+m} Q_{\gamma}(w, \eta) D^{\gamma} F(w)\right| \leq C(1+|w|+|\eta|)^{q} \operatorname{dist}\left(w, \mathcal{N}^{k+1}\right)^{2 \mu^{\prime}}|F|_{p, B_{1}[w]}, \forall w, \eta \in \mathbb{R}^{N} .
$$

Demonstração: Se $\operatorname{dist}\left(w, \mathcal{N}^{k+1}\right) \geq 1$ isso é imediato. Caso contrário, existe $\xi^{*} \in \mathcal{N}^{k+1}$ tal que $\operatorname{dist}\left(w, \mathcal{N}^{k+1}\right)=\left|w-\xi^{*}\right|<1$. No ponto $\xi^{*}$ a função

$$
L(w)=\sum_{|\gamma| \leq k m+k+m} Q_{\gamma}(w, \eta) D^{\gamma} F(w)
$$

se anula de ordem $2 \mu^{\prime}$. Calculando sua fórmula de Taylor em $\xi^{*}$ obtemos:

$$
\begin{gathered}
L(w)=\sum_{|\gamma|<2 \mu^{\prime}} \frac{1}{\gamma !} \partial^{\gamma} L\left(\xi^{*}\right)\left(w-\xi^{*}\right)^{\gamma}+ \\
+2 \mu^{\prime} \int_{0}^{1}(1-t)^{2 \mu^{\prime}-1} \sum_{|\gamma|=2 \mu^{\prime}} \frac{1}{\gamma !} \partial^{\gamma} L\left(\xi^{*}+t\left(w-\xi^{*}\right)\right)\left(w-\xi^{*}\right)^{\gamma} d t .
\end{gathered}
$$

Portanto

$$
\begin{aligned}
|L(w)| & \leq C^{\prime} \operatorname{dist}\left(w, \mathcal{N}^{k+1}\right)^{2 \mu^{\prime}} \sup _{|\gamma| \leq 2 \mu^{\prime}, z \in\left[\xi^{*}, w\right]}\left|D^{\gamma} L(z)\right| \\
& \leq C \operatorname{dist}\left(w, \mathcal{N}^{k+1}\right)^{2 \mu^{\prime}}(1+|w|+|\eta|)^{q} \sup _{|\gamma| \leq p, z \in B_{1}[w]}\left|D^{\gamma} F(w)\right| \\
& \leq C \operatorname{dist}\left(w, \mathcal{N}^{k+1}\right)^{2 \mu^{\prime}}(1+|w|+|\eta|)^{q}|F|_{p, B_{1}[w]} .
\end{aligned}
$$

Lema 8.0.44 Se $F$ é convexo e $g \in \mathcal{C}^{m}\left(\mathbb{R}^{N}\right)$, então existe uma constante $C$ tal que se $x, y \in F$ são distintos e $|\beta| \leq m$,

$$
\frac{\left|R_{m}^{(\beta)}(x, y)\right|}{|x-y|^{m-|\beta|}} \leq C \sum_{|\gamma| \leq m} \sup _{z \in F}\left|D^{\gamma} g(z)\right|
$$

Demonstração: Como vimos anteriormente,

$$
\left|R_{m}^{(\beta)}(x, y)\right| \leq C \int_{0}^{1} \sum_{|\gamma|=m}\left|D^{\gamma} g(y+t(x-y))-D^{\gamma} g(y)\right||x-y|^{m-|\beta|} d t .
$$

Assim, existe uma constante $K$ tal que

$$
\begin{aligned}
\frac{\left|R_{m}^{(\beta)}(x, y)\right|}{|x-y|^{m-|\beta|}} \leq & K \sum_{|\gamma|=m} \sup _{z \in[x, y]}\left|D^{\gamma} g(z)-D^{\gamma} g(y)\right| \\
& \leq 2 K \sum_{|\gamma|=m} \sup _{z \in[x, y]}\left|D^{\gamma} g(z)\right| \\
& \leq 2 K \sum_{|\gamma| \leq m} \sup _{z \in F}\left|D^{\gamma} g(z)\right| .
\end{aligned}
$$


Corolário 8.0.5 Se $F \subseteq \mathbb{R}^{N}$ é convexo e $g \in \mathcal{C}^{m}\left(\mathbb{R}^{N}\right)$, existe uma constante $S>0$ tal que:

$$
|g|_{m, F} \leq S \sup _{|\beta| \leq m, y \in F}\left|D^{\beta} g(y)\right| .
$$

Demonstração: Por definição,

$$
|g|_{m, F}=\sup \left\{\left|D^{\beta} g(y)\right|, \frac{\left|R_{m}^{(\beta)}(y ; z)\right|}{|y-z|^{m-|\beta|}}:|\beta| \leq m ; y, z \in F, y \neq z\right\} .
$$

Como vimos, existe $C>0$ tal que se $y, z \in F, y \neq z,|\beta| \leq m$,

$$
\begin{aligned}
\frac{\left|R_{m}^{(\beta)}(y, z)\right|}{|y-z|^{|\beta|-m}} & \leq C \sum_{|\gamma| \leq m} \sup _{w \in F}\left|D^{\gamma} g(w)\right| \\
& \leq S \sup _{|\gamma| \leq m, w \in F}\left|D^{\gamma} g(w)\right| .
\end{aligned}
$$




\section{Capítulo 9}

\section{Notações}

Vamos listar algumas das notações utilizadas durante este trabalho.

$$
\begin{aligned}
& \mathbb{N}=\{0,1,2, \ldots\} \\
& |\alpha|=\alpha_{1}+\ldots+\alpha_{n}, \text { se } \alpha \in \mathbb{N}^{n} \text { é escrito como } \alpha=\left(\alpha_{1}, \ldots, \alpha_{n}\right) . \\
& \mathcal{S}=\left\{\varphi \in \mathcal{C}^{\infty}\left(\mathbb{R}^{n}\right): \sup _{x \in \mathbb{R}^{n}}\left|x^{\alpha} \partial^{\beta} \varphi(x)\right|<\infty, \forall \alpha, \beta \in \mathbb{N}^{n}\right\} . \\
& F_{m, n}(f)=\sup _{\xi \in \mathbb{R}^{n}}(1+|\xi|)^{n} \sup _{|\beta| \leq m}\left|D^{\beta} f(\xi)\right|, \text { onde } m, n \in \mathbb{N} .
\end{aligned}
$$

$\mathcal{D}(\Omega)=\left\{\varphi \in \mathcal{C}^{\infty}(\Omega): \operatorname{supp} \varphi\right.$ é compacto e $\left.\operatorname{supp} \varphi \subseteq \Omega\right\}$, onde $\Omega \subseteq \mathbb{R}^{n}$ é um aberto.

$\mathcal{D}^{\prime}(\Omega)$ é o conjunto das distribuições: funcionais lineares contínuos definidos em $\mathcal{D}(\Omega)$.

$A_{\xi}=\left\{w \in A:|\xi-w| \leq R=\left(\frac{10}{7}+1\right) \frac{21 \sqrt{n}}{8}\right\}$, onde $A \subseteq \mathbb{R}^{n}$ e $\xi \in \mathbb{R}^{n}$.

$\mathcal{N}^{k}=\cap_{|\alpha|<k}\left\{\xi \in \mathbb{R}^{n}: P^{(\alpha)}(\xi)=0\right\}$, se $P$ é um polinômio em $\mathbb{R}^{n}$.

$$
\begin{aligned}
& f_{m}(\xi ; \eta)=\sum_{|\alpha| \leq m} D^{\alpha} f(\eta) \frac{(\xi-\eta)^{\alpha}}{\alpha !}, \text { se } f \in \mathcal{C}^{m}\left(\mathbb{R}^{n}\right) . \\
& R_{m}(\xi ; \eta)=f(\xi)-f_{m}(\xi ; \eta) \text {, se } f \in \mathcal{C}^{m}\left(\mathbb{R}^{n}\right) . \\
& |f|_{m, A}=\sup C \cup D, \text { se } A \subseteq \mathbb{R}^{n}, f \in \mathcal{C}^{m}\left(\mathbb{R}^{n}\right), \\
& C=\left\{\left|D^{\alpha} f(x)\right|: x \in A,|\alpha| \leq m\right\}, \\
& D=\left\{\frac{\left|R_{m}^{(\alpha)}(x ; y)\right|}{|x-y|^{m-|\alpha|}}: x, y, \in A, x \neq y,|\alpha| \leq m\right\} .
\end{aligned}
$$

$S(x, y)=B_{1}[x] \cup B_{1}[y]$, onde $x, y \in \mathbb{R}^{n}$.

$S_{1}(x, y)=\cup_{w \in[x, y]} B_{1}[w]$, onde $x, y \in \mathbb{R}^{n}$. 


\section{Referências Bibliográficas}

[1] ATIYAH, M.F. Resolution of singularities and division of distributions. Communications on pure and applied mathematics vol. 23, p. 145-150, 1970.

[2] LIMA, E.L. Análise no espaço $\mathbb{R}^{n}$. Rio de Janeiro: Instituto Nacional de Matemática Pura e Aplicada, 2002.

[3] GRAUERT, H. Several complex variables. Berlin: Springer-Verlag, 1976.

[4] GRAUERT, H., FRITZSCHE, K. Several Complex Variables. New York: Springer-Verlag, 1976.

[5] HÖRMANDER, L. The analysis of linear partial differential operators I: distribution theory and Fourier analysis. Berlin: Springer-Verlag, 1983.

[6] HÖRMANDER, L. The anaylsis of linear partial differential operators II: differential operators with constant coefficients. Berlin: Springer-Verlag, 1983.

[7] HÖRMANDER, L. On the division of distributions by polynomials. Arkiv för Matematik, vol.3, n.53, p.555-568, 1958.

[8] KRANTZ, S.G. A primer of analytic functions. Basel: Birkhäuser, 1992. Puiseux series and Separate real analyticity, p. 80-87. (Baster Lehrbücher, 4).

[9] LOJASIEWICZ, S. Sur le problème de la division. Studia Mathematica, vol. 28, p. 87-136, 1959.

[10] SCHWARTZ, L. Division par une fonction holomorphe sur une varieté analytique complexe. Summa Brasil. Math, vol. 3, p. 181-209, 1955.

[11] STEIN, E.M. Singular integrals and differentiability properties of functions. Princeton: Princeton University Press, 1970.

[12] TREVES, F. Hypo-analytic structures: local theory. Princeton: Princeton University Press, 1992.

[13] TREVES, F. Topological vector spaces, distributions and kernels. Mineola: Dover, 1995.

[14] VILLARREAL ALVARADO, F. Teorema de Seidenberg-Tarski e algumas de suas aplicações às equações diferenciais parciais lineares. São Paulo, 1978. Dissertação - Instituto de Matemática e Estatística, Universidade de São Paulo. 